\title{
THE SUMMER MEETING IN KINGSTON
}

The fifty-eighth Summer Meeting and the thirty-fourth Colloquium of the American Mathematical Society were held at Queen's University and the Royal Military College, Kingston, Ontario, Canada, from August 31 to September 5, 1953 in conjunction with meetings of the Canadian Mathematical Congress, the Mathematical Association of America, the Institute of Mathematical Statistics, and the Economic Society.

Over 700 people registered for the meeting, including the following 377 members of the Society:

C. R. Adams, R. B. Adams, J. G. Adshead, M. I. Aissen, B. E. Allen, E. B. Allen, C. B. Allendoerfer, J. P. van Alstyne, S. A. Amitsur, A. G. Anderson, R. D. Anderson, R. G. Archibald, Richard Arens, E. L. Arnoff, H. E. Arnold, K. J. Arnold, Nachman Aronszajn, M. G. Arsove, M. C. Ayer, I. A. Barnett, C. F. Barr, J. H. Barrett, L. K. Barrett, R. C. F. Bartels, R. G. Bartle, P. T. Bateman, W. R. Baum, Samuel Beatty, E. G. Begle, Theodore Bennett, R. H. Bing, D. W. Blackett, David Blackwell, J. R. Blum, M. I. Blyth, Salomon Bochner, H. F. Bohnenblust W. M. Boothby, R. C. Bose, Evelyn Boyd, C. B. Boyer, A. T. Brauer, G. U. Brauer, F. R. Britton, F. E. Browder, F. H. Brownell, G. S. Bruton, F. J. H. Burkett, G. H. Butcher, James William Butler, P. L. Butzer, W. R. Callahan, E. A. Cameron, J. W. Campbell, L. L. Campbell, K. H. Carlson, A. B. Carson, Henri Cartan, C. R. Cassity, Lamberto Cesari, Abraham Charnes, Randolph Church, R. V. Churchill, Paul Civin, C. J. Clark, H. M. Clark, A. B. Clarke, H. E. Clarkson, D. E. Coffey, L. W. Cohen, Harvey Cohn, R. H. Cole, A. J. Coleman, J. B. Coleman, Esther Comegys, R. M. Conkling, T. F. Cope, A. H. Copeland, Sr., H. S. M. Coxeter, J. B. Crabtree, C. C. Craig, H. F. Cullen, A. B. Cunningham, H. B. Curry, A. E. Danese, J. M. Danskin, G. B. Dantzig, R. A. Dean, R. Y. Dean, J. C. E. Dekker, D. B. DeLury, Douglas Derry, W. F. Donoghue, Jr., C. H. Dowker, A. C. Downing, D. W. Dunn, W. L. Duren, Jr., W. F. Eberlein, H. W. Ellis, M. P. Emerson, H. P. Evans, R. L. Evans, Walter Feit, H. H. Ferns, J. V. Finch, D. T. Finkbeiner, Irwin Fischer, E. E. Floyd, G. E. Forsythe, J. S. Frame, Evelyn Frank, H. D. Friedman, A. H. Frink, Orrin Frink, R. M. Frisch, R. E. Fullerton, I. N. Gál, I. S. Gál, H. L. Garabedian, A. O. Garder, Boris Garfinkel, H. M. Gehman, Irving Gerst, Murray Gerstenhaber, K. S. Ghent, Leonard Gillman, Seymour Ginsburg, Sidney Glusman, Herbert Goertzel, Casper Goffman, Michael Goldberg, Harry Gonshor, S. H. Gould, R. L. Graves, J. W. Green, Harold Greenspan, J. A. Greenwood, F. L. Griffin, V. G. Grove, Simon Gruenzweig, Irwin Gutman, P. R. Halmos, M. E. Hamstrom, J. F. Hannan, B. I. Hart, G. E. Hay, Henry Helson, Melvin Henriksen, J. G. Herriot, I. N. Herstein, M. J. Herzberger, Edwin Hewitt, T. H. Hildebrandt, J. G. Hocking, R. V. Hogg, F. E. Hohn, D. L. Holl, M. P. Hollcroft, T. R. Hollcroft, W. A. Hurwitz, W. R. Hutcherson, Jack Indritz, S. L. Isaacson, J. R. Isbell, Arno Jaeger, T. J. Jaramillo, R. L. Jeffery, W. E. Jenner, Meyer Jerison, A. E. Johns, L. W. Johnson, R. E. Johnson, F. E. Johnston, B. W. Jones, P. S. Jones, G. K. Kalisch, L. H. Kanter, Leo Katz, D. E. Kearney, M. E. Kellar, J. B. Kelly, J. R. F. Kent, D. E. Kibbey, T. C. Koopmans, C. F. Kossack, H. L. Krall, Max Kramer, Saul Kravetz, Solomon Kullback, O. E. Lancaster, C. E. Langenhop, E. H. Larguier, J. W. Lawson, C. Y. Lee, H. L. Lee, A. B. Lehman, 
F. C. Leone, Benjamin Lepson, C. A. Lester, W. J. LeVeque, D. J. Lewis, F. A. Lewis, M. M. Lipschutz, Mark Lotkin, R. C. Lyndon, H. T. McAdams, N. H. McCoy, S. W. McCuskey, W. H. McEwen, J. E. McLaughlin, E. J. McShane, R. W. MacDowell, G. W. Mackey, H. M. MacNeille, M. S. Macphail, W. G. Madow, G. R. Magee, Lawrence Markus, Imanuel Marx, K. O. May, Paul Meier, B. E. Meserve, E. A. Michael, Carman E. Miller, Norman Miller, H. D. Mills, J. M. Mitchell, Don Mittleman, E. B. Mode, E. E. Moise, H. F. Montague, M. D. Montgomery, J. C. Moore, J. T. Moore, T. W. Moore, K. A. Morgan, D. C. Morrow, T. A. Mossman, S. T. C. Moy, C. W. Munshower, W. L. Murdock, Zeev Nehari, August Newlander, Jr., D. J. Newman, C. V. Newsom, A. V. Newton, Jerzy Neyman, C. O. Oakley, R. E. O'Connor, Katsumi Okashimo, E. G. Olds, E. M. Olson, J. C. Oxtoby, F. D. Parker, Emanuel Parzen, S. E. Pence, F. W. Perkins, G. M. Petersen, H. P. Pettit, C. G. Phipps, George Piranian, Everett Pitcher, J. C. Polley, Pasquale Porcelli, D. H. Potts, I. R. Pounder, J. H. Powell, G. B. Price, L. E. Pursell, Tibor Rad6, J. F. Randolph, R. B. Rasmusen, L. T. Ratner, G. E. Raynor, H. W. Reddick, R. M. Redheffer, P. K. Rees, R. F. Reeves, Eric Reissner, H. G. Rice, J. D. Riley, E. K. Ritter, Herbert Robbins, M. S. Robertson, G. de B. Robinson, Louis Robinson, Jack C. Rogers, P. G. Rooney, Arthur Rosenthal, Edward Rosenthall, M. F. Rosskopf, S. G. Roth, E. H. Rothe, Herman Rubin, Phyllis Rubin, M. F. Ruchte, J. P. Russell, Arthur Saastad, Charles Saltzer, H. E. Salzer, Hans Samelson, D. E. Sanderson, Leo Sario, H. M. Schaerf, R. D. Schafer, J. A. Schatz, F. J. Scheid, Peter Scherk, E. C. Schlesinger, E. R. Schneckenburger, Lowell Schoenfeld, B. L. Schwartz, W. R. Scott, D. H. Shaftman, Seymour Sherman, A. L. Shields, Edward Silverman, Annette Sinclair, Aubrey H. Smith, F. C. Smith, K. T. Smith, W. K. Smith, D. O. Snow, W. S. Snyder, D. E. Spencer, G. L. Spencer, V. E. Spencer, E. R. Stabler, M. E. Stark, F. H. Steen, C. F. Stephens, B. M. Stewart, A. C. Sugar, D. B. Sumner, Irving Sussman, R. L. Swain, William Clare Taylor, G. H. M. Thomas, G. L. Thompson, D. L. Thomsen, Jr., R. M. Thrall, H. S. Thurston, H. E. Tinnappel, M. M. Torrey, E. A. Trabant, A. W. Tucker, J. W. Tukey, H. L. Turrittin, J. L. Ullman, H. E. Vansant, J. E. Vollmer, M. E. G. Waddell, T. L. Wade, R. W. Wagner, E. B. Walters, S. L. Ward, J. F. Wardwell, W. G. Warnock, J. V. Wehausen, C. P. Wells, J. G. Wendel, F. J. Weyl, G. W. Whitehead, K. B. Whitehead, A. L. Whiteman, G. T. Whyburn, L. S. Whyburn, W. L. G. Williams, L. M. Winer, Clement Winston, F. B. Wright, F. M. Wright, Hidehiko Yamabe, L. C. Young, Arthur Zeichner, A. D. Ziebur, Antoni Zygmund.

The Colloquium Lectures, On the existence and properties of certain singular integrals, were delivered by Professor Antoni Zygmund of the University of Chicago. The meeting opened with the first lecture at which Professor G. T. Whyburn, President of the Society, presided. Professors Tibor Radó, R. L. Jeffery, and Arthur Rosenthal presided at the remaining lectures.

Professor Henri Cartan of the University of Paris presented two lectures on Recent results in the theory of analytical functions of several variables under the joint auspices of the Canadian Mathematical Congress and the Society, Professors G. T. Whyburn and J. S. Frame presiding.

By invitation of the Committee to Select Hour Speakers for Summer and Annual Meetings, Professor Salamon Bochner of Princeton 
University addressed the Society on Fourier transforms in the theory of probability. Professor T. H. Hildebrandt presided.

There were fourteen sessions for contributed papers presided over by Professors Paul Civin, H. B. Curry, Dr. G. E. Forsythe, Professors Murray Gerstenhaber, T. R. Hollcroft, W. A. Hurwitz, F. W. Perkins, George Piranian, Everett Pitcher, G. B. Price, Eric Reissner, Dr. Seymour Sherman, Professors A. L. Whiteman, L. C. Young.

The Council met on Tuesday evening, September 1.

The Secretary announced the election of the following thirty-five persons to ordinary membership in the Society:

Dr. Julian Adem, University of Mexico;

Miss Mary Ruth Arnette, Oak Ridge National Laboratory;

Mr. Robert Waller Bagley, University of Florida;

Dr. George L. Baldwin, Bell Telephone Laboratories, Murray Hill, New Jersey;

Mr. Walter Brunner, Redstone Arsenal, Huntsville, Alabama;

Miss Virginia Clyde Carlock, Oak Ridge National Laboratory;

Professor George Irvin Cohn, Illinois Institute of Technology;

Professor Wilfred James Combellack, Colby College;

Mr. Robert Lloyd Davis, University of Michigan;

Miss Irene L. Doto, Temple University;

Mr. Norbert A. Eisen, Mid Continent Petroleum Corporation, Tulsa, Oklahoma;

Mr. Frank August Engel, Jr., Westinghouse Atomic Power Division, Pittsburgh, Pennsylvania;

Professor Ahmed Cemal Eringen, Illinois Institute of Technology;

Dr. A. E. Foster, Newark College of Engineering;

Mr. James Phillip Frink, Brown University;

Mr. Dean Gillette, University of California, Berkeley;

Mr. Christopher Gregory, University of Hawaii;

Mr. Wayne Hewitt Jones, Department of Defense, Washington 25, D. C.;

Professor Hyman Kaufman, McGill University;

Dr. Kazar Kazarian, Franklin Institute Laboratories, Philadelphia, Pennsylvania;

Professor John William Keesee, University of Arkansas;

Mr. Robert Roy Korfhage, United Aircraft Corporation, East Hartford, Connecticut;

Mr. Karl Kozarsky, U. S. Naval Proving Ground, Dahlgren, Virginia;

Mr. Francis Patrick Larkin, Fordham College;

Mrs. Norma Lee (Ashby) Lindemann, University of Notre Dame;

Dr. John Edward Maxfield, U. S. Naval Ordnance Test Station, China Lake, California;

Mr. Kenneth Roy Mount, University of Illinois;

Mr. Richard Sheldon Palais, Harvard University;

Mr. Carl Henry Pollmar, Engineering Research Institute, University of Michigan;

Dr. Judah Ben Rosen, Forrestal Research Center, Princeton University;

Dr. Thomas Lorie Saaty, Yale University;

Mr. Gerald Sheldon Silberman, University of California, Berkeley;

Mr. Frederick C. Warner, University of Buffalo;

Mr. George Kilbourne White, United Aircraft Corporation, East Hartford, Connecticut;

Professor Kuo-Tai Yen, Rensselaer Polytechnic Institute. 
It was reported that the following persons had been elected as nominees of the institutions indicated:

Brooklyn College: Mr. Kenneth Norman Geller, Mr. Allan Joseph Goldman, and Mr. Joel Lebowitz.

Harvard University: Mr. Harry Gonshor.

Stanford University: Mr. Paul G. Loewner, Mr. Edward Blake McLeod, Jr., Mr.

Grove Crawford Nooney, Mr. Louis Anthony Schmittroth, Mr. Donald Platte

Squier, and Mr. Martin Judy Vitousek.

University of Toronto: Rev. John Christian Egsgard and Mrs. Sudha Jain.

University of Utah: Mr. Harvey Junior Fletcher.

The Secretary announced that the following persons had been admitted to the Society in accordance with reciprocity agreements with the following organizations: Société Mathématique de France: Mr. Aimé Hennequin, Faculté des Sciences, Paris; Indian Mathematical Society: Professor H. G. S. Sharma, Siddaganga Sanscrit College, Dr. Tallamraju Venkatarayudu, Polytechnic Institute of Brooklyn; Svenska Matematikersamfundet: Dr. Gustav Olof Thorin, Valand, Ömsesidigt Livförakringsbolag, Stockholm, Sweden; Wiskundig Genootschap: Professor Dwinger, University of Indonesia.

The following appointments of representatives of the Society were reported: at the inauguration of John Thomas Wahlquist as President of San Jose State College on May 1, 1953: Professor H. M. Bacon; at the inauguration of Jay Frederick Wesley Pearson as President of the University of Miami on May 7, 1953: Mr. H. Raymond Wright; at the 50th anniversary of the Chicago Law School on May 8, 1953: Professor E. J. Moulton; at the dedication of two buildings on the campus of Mary Washington College of the University of Virginia on May 8-9, 1953: Professor E. E. Floyd; at the inauguration of Oliver S. Willham as President of Oklahoma Agricultural and Mechanical College on May 8-9, 1953: Professor O. H. Hamilton; at the inauguration of G. Brooks Earnest as President of Fenn College on May 9, 1953: Professor J. R. Mussleman; at the inauguration of Malcolm A. Love as President of San Diego State College on May 10, 1953: Mr. L. M. Klauber.

The following additional appointments by the President were reported: A. H. Taub appointed as Chairman of the Editorial Committee for Applied Mathematics Proceedings for the period July 1, 1953-June 30, 1954; A. E. Heins appointed a member of the Editorial Committee for Applied Mathematics Symposium Proceedings for a period of three years beginning July 1, 1953 (Committee now consists of A. H. Taub (Chairman), R. V. Churchill, and A. E. Heins); Hassler Whitney as Chairman of the Organizing Committee for Sum- 
mer Institutes for the period July 1, 1953-June 30, 1954; S. S. Chern and F. B. Jones as members of the Organizing Committee for Summer Institutes for a period of three years beginning July 1, 1953 (Committee now consists of Hassler Whitney (Chairman), A. A. Albert, S. Bochner, S. S. Chern, F. B. Jones, and H. P. Robertson); R. P. Boas reappointed Chairman of the Committee on Translations from Russian and other Foreign Languages for the period July 1, 1953-June 30, 1954; J. L. Doob re-appointed as a member of the Committee on Translations from Russian and other Foreign Languages for a period of three years beginning July 1, 1953 (Committee now consists of R. P. Boas (Chairman), R. E. Bellman, J. L. Doob, Irving Kaplansky, and Hans Samelson); M. H. Martin as the Society's representative on the Program Committee in charge of arranging the two conferences co-sponsored by the Committee on Training and Research in Applied Mathematics of the National Research Council and the American Mathematical Society; B. P. Gill and C. H. W. Sedgewick as auditors for 1953 ; C. S. Pettis to replace T. F. Jordan as Chairman of the Arrangements Committee for the meeting to be held at Wofford College, Spartanburg, South Carolina, on November 27-28, 1953 (Committee now consists of C. S. Pettis (Chairman), John Hill, G. May, E. H. Shuler, and W. M. Whyburn); as an arrangements committee for the Annual Meeting to be held at The Johns Hopkins University on December 28-31, 1953: D. C. Lewis (Chairman), W. L. Chow, A. H. Clifford, L. W. Cohen, H. M. Gehman, E. K. Haviland, W. K. Morrill, and Marian M. Torrey; as a committee to advise with the Executive Director on an experimental project to produce the Mathematical Reviews by the photo offset method: William Feller (Chairman), M. R. Hestenes, G. B. Price; as a committee to consider participation on a National Roster of Mathematicians: E. G. Begle (Chairman), D. H. Lehmer, and S. S. Wilks; as a committee to survey the Society's program of periodical publications: G. A. Hedlund (Chairman), S. B. Myers, and J. C. Oxtoby.

The following dates of meetings have been approved by the Council: November 25-26, 1955 at the Milwaukee Extension of the University of Wisconsin. The Council voted to accept an invitation to hold the 1955 Summer Meeting at the University of Michigan, Ann Arbor, Michigan and the 1955 Annual Meeting at the Rice Institute, Houston, Texas.

Invitations to give addresses in 1953 were announced: Paul Erdös at the October meeting in New York City; B. J. Pettis and A. S. Householder at the November meeting in Spartanburg, South 
Carolina; P. C. Rosenbloom at the November meeting in Evanston; M. M. Schiffer at the November meeting in Pasadena, and D. V. Widder and A. D. Wallace at the Annual Meeting in Baltimore.

The Secretary reported that the Trustees had changed the fiscal year, which will now run from June 1 to May 31 .

The Secretary reported that Professor E. J. McShane and R. L. Wilder had been appointed as representatives of the Policy Committee on the United States National Committee for Mathematics of the National Research Council for a period of four years beginning July 1, 1953 and that Professor J. B. Rosser had been appointed by the Policy Committee to represent mathematics on the Committee to evaluate the present functions and operations of the National Bureau of Standards in relation to the present national needs.

The Council voted to approve a contract with the Office of Scientific Research of the Air Research and Development Command under which the Society will administer a panel of reviewers. Members of this panel will be appointed on nomination by the Office of Scientific Research and approval by the Society. Lists of suggested names for this panel will be furnished by the Editorial Committee for the Mathematical Reviews on request by the Office of Scientific Research. Research proposals submitted to the Office of Scientific Research will be reviewed by individual members of the panel selected by the Office of Scientific Research. Reviewers' reports will be sent directly to the Office of Scientific Research. Payments to the reviewers will be made by the Society.

The Executive Director reported increasing difficulties in distribution of our periodicals because of changes in postal regulations and also reported difficulties resulting from late arrival at Headquarters of change of address notices.

The Council adopted the following resolution: "The Society associates itself with Columbia University in supporting the theme of the University's Bicentennial Celebration-Man's Right to Knowledge and the Free Use Thereof, and joins with the University in inviting John von Neumann to address a special session of its April, 1954, meeting in New York in honor of the occasion."

The Council elected Professor H. J. Ettlinger and C. C. MacDuffee as representatives of the Society on the National Research Council for the period July 1, 1954-June 30, 1957 and elected Professor G. A. Hedlund and E. J. McShane as representatives of the Society on the Council of AAAS for 1954-55.

The Council voted to invite Professor Enrico Fermi to deliver the Gibbs Lecture in 1954. 
A Business Meeting was held on Wednesday September 6 at 11:00 A.M., President Whyburn presiding.

The Secretary reported on the affairs of the Society.

Two amendments to the by-laws of the Society were presented with the recommendation of the Council. These amendments would:

1. empower the Council, under certain conditions, to speak in the name of the Society with respect to matters affecting the status, dignity, and effective position of mathematics or mathematicians.

2. require a referendum on such Council actions when requested by 200 or more members of the Society.

3. require a business meeting at the Summer as well as the Annual Meeting.

4. allow final action to be taken at a business meeting only when business is accepted by unanimous consent or notified to the full membership of the Society in the call for the meeting.

It was moved by Professor Radó, and seconded, "To defer final action on these amendments until the text of the amendments, together with a summary of the arguments which had been advanced for and against them, had been sent to the entire membership of the Society, and to invite the membership to express their opinions." It was moved by Professor R. L. Swain, and seconded to amend Professor Radó's motion by adding the phrase "the opinions received to be presented to the Council for their consideration and possible revision of the proposed amendments." Professor Swain's amendment was approved by the members present and the amended motion was carried.

The Committee on Arrangements provided, for the entertainment of those attending the meeting, an enjoyable program including a reception on Monday evening, a tour of the city and Old Fort Henry on Tuesday afternoon, an excursion among the Thousand Islands on Wednesday afternoon, and a theatre party on Thursday evening at which members were guests of the Canadian Mathematical Congress.

The audience at the theatre were constituted a Committee of the Whole, representing the several societies attending the meeting, to receive a resolution thanking Queen's University, the Royal Military College, the Canadian Mathematical Congress and all responsible individuals for their generous and effective contributions to the success of the meeting. The resolution was presented by Professor J. W. Tukey and passed by a unanimous rising vote.

The abstracts of papers presented at the meeting follow. Those with " $t$ " after the abstract number were presented by title. Where a paper, presented in person, has joint authorship, (p) follows the name 
of the author who presented it. Professor Noonan was introduced by Dean W. H. McEwen, Dr. Schwartz by Professor L. W. Cohen, Mr. Snyder by Professor Albert Wilansky, Professor Reza by Professor Norman Levinson, Dr. Gilmore by Professor E. W. Beth, Dr. Wang by Mr. Joel Pitcairn, and Dr. Feidner by Professor Edith R. Schneckenburger.

\section{Algebra AND Theory of Numbers}

\section{7t. A. A. Albert: The structure of right alternative algebras.}

If $\mathfrak{A}$ is a right alternative algebra, the attached commutative algebra $\mathfrak{A}(+)$ is a special Jordan algebra. The author considers the case where the base field $\mathfrak{F}$ is algebraically closed and of characteristic not two and defines a natural "primitive" trace function $\delta(x)$ for $\mathfrak{Q}^{(+)}$with the usual properties as regards the radical. He then shows that $\delta(x y)=\delta(y x)$ for every $x$ and $y$ of $\mathfrak{A}$. The usual trace properties then hold for $\mathfrak{A}$ and there results the theorem which states that a semisimple right alternative algegra over any field of characteristic not two is alternative. (Received June 3,1953.)

558. S. A. Amitsur: Noncommutative cyclic fields.

A cyclic extension $F$ of order $n$ of a sfield $C$ is a sfield $F \supset C$ which possesses a cyclic group of automorphisms of order $n$ leaving $C$ invariant and which is a right $C$-module of dimension $n$. With this definition, the theory of commutative cyclic fields can be extended to the noncommutative case. Let $C[t ; D](C[t ; \rho])$ denote the ring of all noncommutative polynomials in $t$ with coefficients of $C$ defined by the relation: $c t=t c+c D\left(c t=t c+c^{\rho}\right), c \in C$, where $D: c \rightarrow c D\left(\rho: c \rightarrow c^{\rho}\right)$ is a derivation (an automorphism) of $C$. It is shown that all cyclic extensions of degree $p$ of a sfield $C$ of characteristic $p \neq 0$ are isomorphic to quotients $C[t ; D] /\left(t^{p}-t-a\right) C[t ; D]$ for some derivation $D$ of $C$ satisfying among others the condition that $D^{p}-D$ is the inner derivation: $c \rightarrow c a-a c$ of $C$. The automorphism of the extension is the one induced by the mapping; $t \rightarrow t+1$. Inductive results are obtained for extensions of degree $p^{0}$. For the case of characteristic zero and sfields whose centre contain a primitive $n$th root of unit $\zeta_{n}$, the author obtains the result that every cyclic extension of degree $n$ with a centre containing $\zeta_{n}$ is isomorphic to the quotient $C[t ; \rho] /\left(t^{n}-a\right) C[t ; \rho]$ for some automorphism $\rho$ of $C$ satisfying among others the condition that $\rho^{n}$ is the inner automorphism: $c \rightarrow a c a^{-1}$ of $C$. These results are used to construct new examples of noncommutative fields containing a nontrivial group of outer automorphisms. (Received June 22, 1953.)

559t. H. W. Becker: The Euler, Lebesgue, and Kraitchik transforms of rational cuboids. Preliminary report.

See Dickson's History II, pp. 497-501, and M. Kraitchik, Scripta Math. vol. 11 (1945) p. 325. A Diophantine solution of $x^{2}+y^{2}=u^{2}, y^{2}+z^{2}=v^{2}, x^{2}+z^{2}=w^{2}$ depends on $x, y, z, u, v, w=e g\left(g^{2}-h^{2}\right), 2 e f g h, g h\left(e^{2}-f^{2}\right), e f\left(g^{2}+h^{2}\right), g h\left(e^{2}+f^{2}\right), \quad\left\{\left(e^{2} g^{2}+f^{2} h^{2}\right)\right.$ - $\left.\left(e^{2} h^{2}+f^{2} g^{2}\right)-4 e^{2} f^{2} g^{2} h^{2}\right\}^{1 / 2}$. Under the Euler transform $E: e, f, g, h \rightarrow e \pm f, g \pm h$; and $x, y, z, u, v, w \rightarrow y z, x z, x y, u z, v x, w y$. Under the Lebesgue transform $L: e, f, g, h \rightarrow e, f$, $x u$, wy; and $x, y, z, u, v, w \rightarrow x^{4}-y^{2} z^{2}=u^{2} x^{2}-w^{2} y^{2}=w^{2} x^{2}-u^{2} z^{2}, 2 u x w y, 2 u x w z, u^{2} x^{2}$ $+w^{2} y^{2}, 2 u x w v, w^{2} x^{2}+u^{2} z^{2}=X, Y, Z, U, V, W$. There are analogous transforms $L^{\prime}$ and $L^{\prime \prime}$, under permutations $(x z)(u v)$ and $(x y)(v w)$ on $L$. Under the Kraitchik transform $E L^{\prime \prime}=K^{\prime \prime}: e, f, g, h \rightarrow v x, u y, u z, v y$; and $x, y, z, u, v, w \rightarrow z\left(y^{4}-x^{2} z^{2}\right), 2 u v x y z$, $x\left(y^{4}-x^{2} z^{2}\right), z\left(v^{2} x^{2}+u^{2} y^{2}\right), x\left(u^{2} z^{2}+v^{2} y^{2}\right), w\left(y^{4}-x^{2} z^{2}\right)=Y^{\prime \prime} z, X^{\prime \prime} z=Z^{\prime \prime} x, \quad Y^{\prime \prime} x, U^{\prime \prime} z$, 
$V^{\prime \prime} x, Y^{\prime \prime} w$. Similarly, under $E L=K$ and $E L^{\prime}=K^{\prime}: x, y, z, u, v, w \rightarrow Y z=Z y, X z, X y$, $U z, X v, W y$, and $Z^{\prime} y, Z^{\prime} x, X^{\prime} y=Y^{\prime} x, Z^{\prime} u, V^{\prime} x, W^{\prime} y$. After eancellations, $E^{2}=I$, $L E=L, K E=K$, etc., but $E K=L$ though $E L=K, L K=L^{2}$ though $K L=K^{2}$, etc. The classic Saunderson solution is $e, f, g, h=r^{2}+s^{2}, 2\left(r^{2}-s^{2}\right), r^{2}+s^{2}, 4 r s$. Another is due to Rignaux "avec l'aide de M. Crussol," L'Int. des Math. vol. 25 (1919) p. $127: e, f, g, h$ $=2\left(r^{2}-r s-s^{2}\right), r^{2}+s^{2}, r(r+2 s), s(2 r-s)$, wherein $r, s \rightarrow r \pm s$ makes $e, f, g, h \rightarrow e \pm f$, $g \pm h$, so operating on this solution $E \subset I$ the identity. Rignaux forms cover nos 4, 15, $16,38,46,49$ of Kraitchik's table of 50 maverick empirical solutions; the other 44 should suggest new formulations. Do there exist: (a) Absolute mavericks? (b) A finite, or infinite, number of 2 parameter solutions of $w=\omega(e, f, g, h)$ ? (Received July 20, 1953.)

560t. Volodymyr Bohun-Chudyniv: On a general method for constructing orthogonal square matrices of every order with differing integers.

In this author's last two papers: (1) On orthogonal and non-orthogonal closed systems of $K$-nions and their application, (2) On a method of determining orthogonal square matrices with differenting integers (also complex numbers, quaternions, octonions, sedecimions, etc.) he considered methods constructed by the author for determining orthogonal square matrices of $2^{k}, 2^{k}-1 ;\left(2^{1+\lambda}-1\right) 2^{\beta}(k \geqq 2 ; \lambda \geqq 1, \beta \geqq 1 ; \lambda \geqq 0, \beta \geqq 2)$ orders. (Bull. Amer. Math. Soc. Abstracts 58-6-621, 59-3-232.) In the present paper the author determines: (A) The general method of constructing orthogonal square matrices of every order composed of differing integers, complex numbers, etc., with the help of expressions (I) constructed by this author: (I) $2 x_{\alpha} x_{1}, 2 x_{\alpha} x_{2}, \cdots, 2 x_{\alpha}^{2}$ $-\sum_{i=1}^{i=n} x_{i}^{2}, 2 x_{\alpha} x_{\alpha+1}, \cdots, 2 x_{\alpha} x_{n}(\alpha=1,2, \cdots, n ; n \geqq 2)$. Expressions (I) can also be obtained from the author's orthogonal schemes of $K$-nions of $2^{k+2}=n+\gamma\left(2^{k+1}>\gamma \geqq 0\right)$ rank as a separate case. The matrices of this type can be made to take on a symmetrical form. (B) The method of constructing orthogonal square matrices with the help of expressions (I) and two lemmas pointed out in the mentioned paper (2) of this author. The largest number of differing elements in type (A) matrices can equal $n(n+1) / 2$ and in type (B) matrices can equal $n(n+2) / 2$ ( $n$ is the order of the matrix). (Received July 14, 1953.)

\section{1t. A. T. Brauer: A new class of Hadamard determinants.}

Recently O. Perron [Math. Zeit. vol. 56 (1952) pp. 122-130] raised the following question. Let $m$ be a composite number of form $4 n-1$. Do there exist matrices with $4 n-1$ rows and $2 n$ columns whose elements are the numbers of a complete system of residues $(\bmod m)$ and which have the following two properties: I. Each row has exactly $n$ elements in common with each other row. II. The corresponding elements of each pair of two rows have the same difference $(\bmod m)$ ? Perron proved that such a matrix exists for $m=15$. Using a theorem of the author on the distribution of the Jacobian symbols [Bull. Amer. Math. Soc. Abstract 59-1-37] it is shown in this paper that such matrices exist for $m=p(p+2)$ if $p$ and $p+2$ are twin primes. It follows from this result that it is possible to construct Hadamard determinants of order $(p+1)^{2}$ if $p$ and $p+2$ are twin primes. (Received July 14,1953 .)

562. A. T. Brauer: On the location of the characteristic roots of a matrix.

The main result of the paper is the following theorem. Let $A=\left(a_{r s}\right)$ be a square matrix of order $n$. Let $D_{r s}$ be the principal two-rowed minor of the characteristic determinant of $A$ which contains elements of the $r$ th and $s$ th rows. If $Q_{r s}$ denotes the 
$\operatorname{sum} \sum_{\nu}\left\{\left|a_{r s} a_{s \nu}\right|+\left|a_{s r} a_{r \nu}\right|+\left|a_{r \nu} a_{s \nu}\right|\right\}+\sum_{\nu, \mu}\left|a_{r \nu} a_{s \mu}+s_{s \nu} a_{r \mu}\right|$ where $\nu$ and $\mu$ run from 1 to $n$ with $\nu<\mu$, and where $\nu \neq r, s$ and $\mu \neq r, s$, then each characteristic root of $A$ lies in the interior or on the boundary of at least one of the $n(n-1) / 2$ ovals of Cassini $\left|D_{r s}\right| \leqq Q_{r s}(r, s=1,2, \cdots, n ; r \neq s)$. This theorem improves former results of the author [Duke Math. J. vol. 13 (1946) pp. 387-395; vol. 14 (1947) pp. 21-26; vol. 15 (1948) pp. 871-877; vol. 19 (1952) pp. 75-91; vol. 19 (1952) pp. 553-562]. (Received July 14, 1953.)

563t. G. C. Byers: Class number relations for quadratic forms over $G F[q, x]$.

Analogues of certain of Kronecker's classic class number relations are obtained for quadratic forms with coefficients from $G F[q, x]$. This is done by establishing a relation between definite binary quadratic forms and associated quaternary bilinear forms. Defining $h(A), A \in G F[q, x]$, to be the number of classes of quadratic forms of discriminant $A$, we quote a typical result. Given $\nabla \in G F[q, x]$ of degree $2 m+1$, then $\sum_{R} h\left(R^{2}-\nabla\right)=2 \sum_{J} q^{j}(j=\operatorname{deg} J)$, where the summation on the left is over all $R$ $\in G F[q, x]$, including $R=0$, of degree not exceeding $m$, while the summation on the right is over those primary $J \in G F[q, x]$ of degree greater than $m$ dividing $\nabla$. Similar results are obtained for $\nabla$ of even degree. (Received May 29, 1953.)

564t. Leonard Carlitz: Pairs of quadratic equations in a finite field.

If $Q\left(\xi_{1}, \cdots, \xi_{r}\right), Q_{r}\left(\xi_{1}, \cdots, \xi_{r}\right)$ denote quadratic forms with coefficients in $G F(q)$, consider the number of solutions of the system $Q_{1}=\alpha, Q_{2}=\beta$. If the $Q$ 's satisfy certain conditions, explicit results are obtained. In some cases the number of solutions is exhibited in terms of Jacobsthal sums. (It may be noted that the case $q=2^{n}$ is included.) (Received May 13, 1953.)

565t. Leonard Carlitz: Representations by quadratic forms in a finite field.

Let $A$ be a symmetric matrix with elements in $G F(q), q$ odd, of order $m$ and nonsingular; let $B$ be symmetric of order $t$ and rank $r$. The principal result of the paper is the determination of $N_{r}(A, B)$, the number of $m \times t$ matrices $X$ such that $X^{\prime} A X=B$. (Received May 13, 1953.)

566t. Leonard Carlitz: The number of solutions of some special equations in a finite field.

Explicit formulas for the number of solutions of certain special equations $f(\xi)=\alpha$ are obtained. In particular such results are obtained in the case $f$ equal to a Pfaffian or a factorable polynomial as well as various combinations of these and other polynomials. In most of the cases discussed the number of solutions satisfies $N_{f}(\alpha)=N_{f}(1)$ for all $\alpha \neq 0$. (Received May 13, 1953.)

\section{7t. F. Marion Clarke: An extension of the n-step gradient method.}

Given $n$ linear equations in $n$ unknowns represented by the vector equation $D x+c$ $=0$, the matrix operator $D$ is called $G$-Hermite if (i) the elements of $D$ belong to a finite cyclic extension $K$ of a base field $F$ and (ii) there exists a unitary matrix $R$ whose elements are relative automorphisms of $K$ over $F$ such that "transpose $R D=D$." It is shown that when $D$ is nonsingular and $R x \cdot x>0$, the Stiefel $n$-step gradient method (Zamp vol. 3 (1952) pp. 1-33) can be generalized to yield the solution in $K$ of the given 
system of equations. The extension uses $G$-Hermite forms and the lemma: if the vectors $x_{1}, x_{2}, \cdots, x_{n}$ with coefficients in $K$ have the property that $R x_{i} \cdot x_{j}=0$ for all $i \neq j$ and $R x_{i} \cdot x_{i} \neq 0$, then $x_{1}, x_{2}, \cdots, x_{n}$ are linearly independent over $F$. It is shown that the method applied with a $G$-Hermite matrix includes the familiar Hermite matrix as a special case. (Received July 21, 1953.)

\section{8t. C. W. Curtis: On the structure of non-semisimple algebras.}

The following extension of the Wedderburn-Malcev theorem for associative algebras is proved. Let $A$ be an algebra over a field $K$ with Jacobson radical $N$, such that $A / N$ is finite-dimensional and separable over $K$. If $\bigcap_{i=1}^{\infty} N^{i}=(0)$, and if $A$ is complete in the natural topology determined by the powers of $N$, then there exists a subalgebra $B$ of $A$ with the following properties: (i) $A=B+N$ (vector space direct sum); and (ii) if $B^{\prime}$ is a separable subalgebra of $A$, then there exists an element $z \in N$ such that if $z^{\prime}$ is the quasi-inverse of $z$, then the mapping $b^{\prime} \rightarrow b^{\prime}-z b^{\prime}-b^{\prime} z^{\prime}+z b^{\prime} z^{\prime}$ is an isomorphism of $B^{\prime}$ into $B$. Consider now a complete semi-local ring $R$ contained in the center of a ring $S$. A sufficient condition is derived in order that $S$ be complete in the topology determined by the powers of its radical. These results yield a theorem on the structure of a ring $S$ which is a finitely generated module over a complete local ring contained in its center. (Received June 15, 1953.)

\section{9t. R. P. Dilworth: Proof of a conjecture on finite modular lattices.}

It is proved that in a finite modular lattice the number of meet irreducibles is equal to the number of join irreducibles. (Received July 20,1953.)

\section{0t. D. O. Ellis: Notes on the foundations of lattice theory. II.}

Let $L$ be a lattice. A mapping $f: L \otimes L \rightarrow S$, where $S$ is an arbitrary set, is called a $c$-function on $L$ provided $f(a, b)=f(a \wedge b, a \bigvee b)$. The notion of $c$-function is a unifying concept in lattice theory. Relations of $c$-functions to valuations, distance spaces, lattice-ordered algebras, and algebraic identities are discussed. Examples of results are: 1. A lattice $L$ is distributive if and only if there is a c-function $f(a, b)$ on $L$ which is biuniform in $b$ for fixed a. 2. A lattice $L$ is modular if and only if there is a c-function $f(a, b)$ on $L$ so that $f(a, b)=f(a, c)$ implies $b=c$ or $b \| c$ (" $b$ and $c$ are incomparable"). The methods are lattice-theoretic and quite elementary. (Received July $3,1953$. )

571. Irwin Fischer: Singular points on the moduli-variety for hyperelliptic curves.

Let $k$ be any algebraically closed ground field. It is shown that one can construct, in a natural manner, an algebraic variety $M$ which, except for an algebraic subvariety, is in one-one correspondence with the birational classes of hyperelliptic curves of genus $\mathrm{g}$, defined over $k$. The question of singular points on $M$ is then investigated. If $g$ is greater than 2, a point $P$ is a singular point of $M / k$ if and only if the function field of the birational class of hyperelliptic curves which $P$ represents possesses at least one nonidentical $k$-automorphism. If $g=2$, a singular point on $M$ is exhibited. (Received July $17,1953$. )

572. J. S. Frame(p), G. de B. Robinson, and R. M. Thrall: The hook graphs of the symmetric group.

To each irreducible representation [ $\lambda]$ of the symmetric group $S_{n}$ corresponds a Young diagram having $\lambda_{i}$ nodes in the $i$ th row and $\lambda_{j}^{\prime}$ nodes in the $j$ th column. The 
$i j$-node is the corner of a right hook of length $h_{i j}=1+\left(\lambda_{i}-j\right)+\lambda_{j}^{\prime}-i$. If $h_{i j}=k q$ and $\lambda_{i}-i+1 \equiv j-\lambda_{i}^{\prime} \equiv r(\bmod q)$, the $i j$-node is called a $q$-node of residue $r$ or a $(q, r)$ node. The hook graph of $[\lambda]$ is an array $H[\lambda]$ obtained by replacing each node of $[\lambda]$ by its hook number $h_{i j}$. The hook product $H_{\lambda}$ is the product of all the $h_{i j}$. The degree $f_{\lambda}$ of $[\lambda]$ is found to be $n ! / H_{\lambda}$. For any positive integer $q$, a $q$-quotient graph $[\lambda]_{q}$ can be constructed very simply from $[\lambda]$ by deleting all nodes except the $q$-nodes. Those of residue $r$ form one of the disjoint constituents of $[\lambda]_{q}$. The hook numbers of $[\lambda]_{q}$ are $h_{i j} / q$. The $q$-quotient graph is closely related to the star diagram used by Robinson, Staal, Nakayama and Osima, and Nagao. Simple proofs of the theorems of Staal [Star diagrams and the symmetric group, Canadian Journal of Mathematics vol. 2 (1950) pp. 79-92] and other important properties of $[\lambda]$ follow immediately from the use of the hook graphs. (Received July 20,1953.)

\section{3t. B. R. Gelbaum: Symmetric games.}

(This work was supported by the Office of Naval Research.) The characteristic function of any symmetric zero-sum $n$-person game can be defined by giving its values $v_{q}$ on sets with $q$ elements. This paper discusses properties of solutions based on the "most efficient coalitions" in such games. These coalitions are defined by setting $a=\max \left\{v_{q} / q\right.$ for $\left.q>0\right\}$ and $p=\min \left\{q\right.$ with $\left.v_{q}=q a\right\}$. The coalitions with $p$ members are said to be most efficient. If one sets $n=p m+r$ with $0 \leqq r<p$, it is shown that: (1) $r>0$. (2) $v_{q}=q a$ if and only if $p$ divides $q \neq 0$. (3) $v_{k}=-k$ implies $k \leqq r$. (4) $1 /(n-1)$ $\leqq a \leqq r / p m$. The main result is to give solutions for those games in which $v_{r}=-r$ and $v_{r+1} \geqq a[r-(n-p+1) /(p-1)]$. For these games, the permutations of the imputations $\alpha^{0}=\left(\alpha_{1}^{0}, \cdots, \alpha_{n}^{0}\right)$ and $\alpha^{1}=\left(\alpha_{1}^{1}, \cdots, \alpha_{n}^{1}\right)$ with $\alpha_{1}^{0}=a$ for $1 \leqq i \leqq p m, \alpha_{1}^{0}=-1$ for $p m+1 \leqq i \leqq n, \alpha_{1}^{1}=a$ for $1 \leqq i \leqq n-p+1, \alpha_{1}^{1}=-((n-p+1) /(p-1)) a$ for $n-p+2$ $\leqq i \leqq n$ form a solution. If $2(p-r)-2 \leqq p \leqq 2(p-r)-1$ then the condition on $v_{r+1}$ can be realized; however there exist cases for which it cannot be realized: This method provides symmetric zero-sum $n$-person games with finite symmetric solutions for a large class of integers $n$ (in particular, for all odd integers). (Received July 20,1953.)

\section{4t. T. R. Hollcroft: On sums of powers of $n$ consecutive integers.}

Let $S_{p}=1^{p}+2^{p}+3^{p}+\cdots+n^{p}$, in which $n$ and $p$ are any finite positive integers. The well known relation, $S_{3}=S_{1}^{2}$, led the author to search for a more general relation (Theorem I below) of which this would be a special case. After obtaining general formulas for $S_{p}$, it is shown that for $p$ odd, $S_{p}$ can be expressed as an algebraic polynomial in $N=n(n+1)$, and for $p$ even, $S_{p}$ can be expressed as the product of $(2 n+1)$ and an algebraic polynomial in $N$. Since $N=2 S_{1}$ and $N(2 n+1)=6 S_{2}$, the following theorems result: Theorem I. For $p$ odd, $S_{p}$ can be expressed as an algebraic polynomial in $S_{1}$. Theorem II. For $p$ even, $S_{p}$ can be expressed as the product of $S_{2}$ and an algebraic polynomial in $S_{1}$. Examples: $S_{11}=S_{1}^{2}\left(16 S_{1}^{4}-32 S_{1}^{3}+34 S_{1}^{2}-20 S_{1}+5\right) / 3$; $S_{10}=S_{2}\left(48 S_{1}^{4}-80 S_{1}^{3}+68 S_{1}^{2}-30 S_{1}+5\right) / 11$. Similar theorems are found for sums of powers of consecutive integers beginning with any positive integer and for sums of powers of consecutive even or odd positive integers. (Received August 31, 1953.)

\section{R. E. Johnson: Semi-prime rings.}

A ring $R$ is semi-prime if the intersection of the prime ideals of $R$ is zero. Prime right ideals may be defined in a semi-prime ring $R$ much as they are in a prime ring (Prime rings, Duke Math. J. vol. 18 (1951) pp. 799-809). The algebra $\mathfrak{P}$ of all prime right ideals of $R$ forms a structure of $R$ in that: (1) $0, R \in \mathfrak{B}$; (2) $\left(I \cap I^{\prime}\right)^{*}=I^{*} \cap I^{*}$; (3) $(I: a)^{*}=\left(I^{*}: a\right)$; where $a \in R, I, I^{\prime}$ are right ideals of $R$, and $I^{*}$ is the least element 
of $\mathfrak{P}$ containing $I$. In general, any subalgebra of $\mathfrak{P}$ satisfying (1)-(3) is called a structure of $R$. If the structure $\Re$ of $R$ has minimal nonzero elements (atoms), $\Re$ is called an $a$-structure. Associated with each semi-prime ring $R$ is a universal extension ring $N(R)$ containing $R$ as an ideal and having the property that the annihilator of $R$ in $N(R)$ is zero. The main theorem of the paper is that $R$ is a semi-prime ring with $a$ structure if and only if $B \oplus B^{\prime} \subseteq R \subseteq N(B) \oplus N\left(B^{\prime}\right)$, where $B$ is a direct sum of prime rings each having an $a$-structure and $B^{\prime}$ is a semi-prime ring having a nonatomic structure. (Received June 3, 1953.)

\section{G. K. Kalisch: m-quota games and m-quota solutions.}

(This work was supported by the Office of Naval Research.) An $m$-quota $n$-person game $Q_{n ; m}$ (for $Q_{n ; 2}$ see L. Shapley, Contributions to the theory of games, II) has the property that there exist numbers $\omega_{i}(i=1, \cdots, n)$ such that (1) $\sum_{k=1}^{m} \omega_{i_{k}}$ $=v\left(\left(i_{1}, \cdots, i_{m}\right)\right)$ (see $\mathrm{v}$. Neumann and Morgenstern, Theory of games $\cdots$, for notations), (2) $\Omega=\sum_{i=1}^{n} \omega_{i}=0$, (3) $\omega_{i} \geqq-1$. Let there be defined integers $b_{i}^{i}$ $(i=1, \cdots, n ; j=2, \cdots, m)$ such that the sets $\left(i, b_{i}^{2}, \cdots, b_{i}^{m}\right)$ each contain $m$ distinct elements. Define the sets of imputations $\lambda_{i}=\left[\left\{\omega_{1}, \cdots, \omega_{i}-\sum_{2}^{m} x_{k}, \cdots, \omega_{b 1}^{2}\right.\right.$ $\left.\left.+x_{2}, \cdots, \omega_{b_{i}^{m}}^{m}+x_{m}, \cdots, \omega_{n}\right\}\right]$. Then for suitable $b_{i}^{j}$, and $v, \Lambda=\bigcup_{i=1}^{m} \lambda_{i}$ is a solution of $Q_{n ; m}$. It is possible to weaken (1)-(3) in various ways and still have a solution of the type above. (Received July 20, 1953.)

577t. J. Lambek and Leo Moser: Elementary methods in the theory of distribution of primes.

Let $h(a)=\sum_{r \leqq a} 1 / r$ and $l_{k}(a)=h(k a)-h(k)$. Using finite manipulations with rationals only the following are proved: 1 . For $k \geqq n \geqq 2, .6<\pi(n) / l_{k}(n)<2$. This improves results of T. S. Broderick [J. London Math. Soc. vol. 14 (1939) pp. 303-310] and F. Behrend [Proc. Roy. Soc. New South Wales vol. 25 (1942) pp. 169-174]. 2. $\left|\sum_{p \leqq a} l_{k}(p)+\sum_{p . q \leqq a} l_{k}(p) l_{k}(q)-2 a l_{k}(a)\right|<61 a+18 a^{3} / k$. This is an analogue of the fundamental lemma of A. Selberg [Ann. of Math. vol. 50 (1949) pp. 305-313]. 3. For each $n>1$, there is a number of the form $p$ or $p \cdot q(p \neq q)$ between $n$ and $n(1+100 / h(n))$. (Received July 16, 1953.)

578t. J. Lambek and Leo Moser: Inverse and complementary functions.

Two arithmetic functions $f$ and $g$ are defined to be inverse if for every pair of positive integers $m, n$, either $f(m)<n$ or $g(n)<m$ but not both. It is shown that two nondecreasing functions $f$ and $g$ are inverse if and only if the sequences $n+f(n)$ and $n+g(n)$ are complementary. Thus it turns out that the sequences $[n+\log n]$ and $\left[n+e^{n}\right]$ are complementary. Also, for irrational $\theta$, the sequences $[n(1+\theta)]$ and $[n(1+1 / \theta)]$ are complementary. This is a result of S. Beatty. Methods for finding the $n$th term of the complement of a given sequence are developed. Thus the $n$th positive integer which is not a $k$ th power is $n+\left[\left(n+\left[n^{1 / k}\right]\right)^{1 / k}\right]$. The $n$th nonprime is the limit of the sequence: $n, n+\pi(n), n+\pi(n+\pi(n)) \cdots$. A method due to V. Brün [Norsk. Mat. Tidsskr. vol. 13 (1931) pp. 73-79] for computing the $n$th prime is generalized. (Received July 16, 1953.)

579t. J. Lambek and Leo Moser: On the distribution of pythagorean triangles.

Estimates are obtained for the number of relatively-prime lattice points in certain 
regions which may extend to infinity. These are used to extend some results of D. N. Lehmer [Amer. J. Math. vol. 22 (1900) pp. 293-335] and D. H. Lehmer [Bull. Amer. Math. Soc. vol. 54 (1948) pp. 1185-1190] on the number of primitive pythagorean triangles satisfying various conditions. Thus, for example, it is shown that the number of primitive pythagorean triangles with area not exceeding $n$ is $c n^{1 / 2}+O\left(n^{1 / 3}\right)$ where $c=\Gamma(1 / 4)^{2} 2^{-1 / 2} \pi^{-5 / 2}=.531340 \cdots$. This agrees well with recently completed tables of F. L. Miksa. These tables list all primitive pythagorean triangles with area less than $10^{\circ}$. (Received July 16, 1953.)

\section{W. J. LeVeque: The distribution of values of multiplicative functions.}

Let $r_{2}(m)$ be the number of representations of $m$ as a sum of two squares, and let $\tau(m)$ be the number of divisors of $m$. Let $R_{k}(x)$ and $T_{k}(x)$ be the number of $m \leqq x$ such that $r_{2}(m)=k$, and $\tau(m)=k$, respectively. Complicated asymptotic estimates are obtained for $R_{k}(x)$ and $T_{k}(x)$; that for $R_{k}(x)$ disproves a conjecture of P. Levy. (Received July $15,1953$.

\section{D. J. Lewis: Polynomial functions over the residue rings $\mathfrak{O} / \mathfrak{p}^{m}$.}

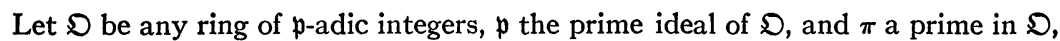
hence $\mathfrak{p}=\pi \mathfrak{D}$. Suppose $\mathfrak{D} / \mathfrak{p}^{m} \cong G F(q)$. Let $\mathbb{B}_{m}=\left\{f\right.$ in $\mathfrak{D}[x]$ such that $\left.f: \mathfrak{D} \rightarrow \mathfrak{p}^{m}\right\}$. It is well known that $\mathcal{B}_{1}=\left(x^{q}-x, \pi\right)$ and this has been most useful in considering functions over finite fields. It is shown that there exist polynomials $\lambda_{m}$ in $\mathfrak{D}[x]$ such that $\mathcal{B}_{m}=\left(\lambda_{m}, \pi \mathcal{B}_{m-1}\right)$. A similar result is obtained for the case of several variables. These facts are used in considering functions over the residue ring $\mathfrak{O} / \mathfrak{p}^{m}$ and some results analogous to those for finite fields are obtained. (Received July 9, 1953.)

\section{2t. L. J. Mordell: On intervals containing an affinely equivalent} set of $n$ integers $\bmod k$.

L. Redei, improving an old result of Thue, has recently proved that if $k$ is a prime, there exist integers $a, b$ with $(a, k)=1$ such that a set of integers $(y)$ defined by $y_{r} \equiv a x_{r}+b(\bmod k)(r=1,2, \cdots, n)$ lies in an interval of width $L$ where $n L^{n-1}$ $\leqq 2^{n-1} k^{n-2}$. The paper contains a simpler proof and also other estimates for $L$ found by using Dirichlet's pigeonhole result. (Received July 9, 1953.)

583t. D. C. Murdoch: On the automorphism group and endomorphism ring of an Abelian group.

The quasi-regular elements of any ring $R$ form a group $\mathfrak{Q}^{*}$ under the operation $x^{*} y=x+y+x y$. Every ideal $B_{1}$ of $R$ intersects $\mathfrak{Q}^{*}$ in a normal subgroup $\mathfrak{S}\left(B_{1}\right)$ of $\mathfrak{Q}^{*}$, while every subgroup $\mathfrak{S}_{1}$ of $\mathfrak{Q}^{*}$ generates an ideal $B\left(\mathfrak{S}_{1}\right)$ in $R$. The normal subgroup $\mathfrak{S}\left[B\left(\mathfrak{S}_{1}\right)\right]$ is called the closure of $\mathfrak{S}_{1}$, and the ideal $B\left[\mathfrak{S}\left(B_{1}\right)\right]$ the closure of $B_{1}$. A subgroup (ideal) which is equal to its closure is closed. The closed subgroups of $\mathfrak{Q}^{*}$ and the closed ideals of $R$ form isomorphic lattices under $\mathfrak{S} \leftrightarrow B(\mathfrak{Q})$. If $R$ is the endomorphism ring of an Abelian group $\mathfrak{B}, \mathfrak{Q}^{*}$ is isomorphic to $\mathfrak{A}$, the automorphism group of $\mathbb{B}$, and the concept of closed subgroup is extended to $\mathfrak{A}$ via this isomorphism. If $\mathfrak{A}_{1}$ is a subgroup of $\mathfrak{A}_{1} \mathfrak{S}_{0}\left(\mathfrak{A}_{1}\right)=\mathbb{S}$, and $\mathfrak{H}_{i}\left(\mathfrak{U}_{1}\right)$ is the group generated by all elements $g^{-1} g^{s}, g \in \mathbb{S}_{i-1}\left(\mathfrak{A}_{1}\right)$ and $s \in \mathfrak{A}_{1}(i=1,2,3, \cdots)$, then $\mathbb{B}$ is $\mathfrak{A}_{1}$-nilpotent if $\mathfrak{S}_{r}\left(\mathfrak{A}_{1}\right)=1$ for some integer $r$. If the minimum condition for left ideals holds in $R$, then $\mathscr{A}$ contains a unique maximal closed subgroup $\mathfrak{A}_{N}$ such that $\mathbb{S}$ is $\mathfrak{A}_{N}$-nilpotent. $\mathfrak{A}_{N} \cong \mathfrak{G}(N)$ where 
$N$ is the radical of $R$ and $\mathfrak{A} / \mathfrak{A}_{N}$ is isomorphic to the direct product of the quasi-regular groups of the simple ideals of $R / N$. (Received July 20,1953.)

584. Bernard Noonan: The construction of faithful representations of Lie algebras of characteristic zero.

This paper gives an explicit procedure for the construction of a faithful representation $S$ of a Lie algebra $L$, over an algebraically closed field of characteristic zero, from a given faithful representation $Q$ of an ideal $T$ of $L$, where $T$ is in the radical $R$ of $L$ and where $Q$ induces a nil representation on $T_{1}$, the intersection of the ideal $L o L$ ( $o$ meaning Lie multiplication) and the radical $R$, and where, furthermore, $Q$ is invariant under $V(L=V+R$ by the theorem of Levi). $Q$ is defined to be invariant under $V$ if, for any $t$ in $T$, and $v$ in $V$, there exists a matrix $C(v)$ such that $C(v) o Q(t)=Q(v o t)$. The representation $S$ so constructed possesses great generality in that one arbitrary eigenvalue can be assigned to the representation of each basis element of the radical $R$ not in $T$. The theorem of Ado is proved as a corollary, using the Birkhoff representation of a nilpotent Lie algebra. (Received July $17,1953$. )

\section{5t. Everett Pitcher: $A$ class of group extensions.}

A system $(H, \phi, \iota)$ is called an $\alpha$-extension of $\alpha: N \rightarrow E$ if $N, E, H$ are topological abelian groups, $\alpha$ is a homomorphism, and (i) $\phi: H \rightarrow E$ is an open homomorphism onto, (ii) $\iota: N \rightarrow H$ is an isomorphism (into), (iii) $\phi \iota=\alpha$, (iv) $\phi^{-1} \alpha N=\iota N$. This reduces to the usual definition of abelian extension when $\alpha$ is trivial. (With an additional hypothesis, non-abelian extensions could be considered.) The existence of $\alpha$-extensions may be regarded as the question of existence of extensions of $E$ by $\alpha^{-10}$ which, through $N$, are partially prescribed. It may be regarded as a problem in inverting the order of homomorphism onto and isomorphism into, with $\alpha: N \rightarrow \alpha N, \alpha N \subset E$ replaced by $\iota: N \rightarrow H, \phi: H \rightarrow E$. Two $\alpha$-extensions $\left(H_{s}, \phi_{s}, \iota_{s}\right), s=1,2$, are $\alpha$-equivalent if there is an isomorphism $\omega: H_{1} \rightarrow H_{2}$ which is onto and for which $\phi_{2} \omega=\phi_{1}$ and $\omega \iota_{1}=\iota_{2}$. Theorem: (a) If $E$ is discrete or if $N$ is compact and $E$ is locally compact, there exist $\alpha$-extensions. (b) The set of $\alpha$-extensions can be put into one-to-one correspondence with the set of extensions of $E / \alpha N$ by $\alpha^{-1}$. (Received July 20,1953.)

\section{6t. Irving Reiner: Symplectic modular complements.}

An integral $(j+k) \times 2 n$ array $(j \leqq n, k \leqq n)$ with first $j$ rows $(A B)$ and next $k$ rows $(C D)$ is called a normal $(j, k)$ array if $A B^{\prime}$ and $C D^{\prime}$ are symmetric, and $A D^{\prime}-B C^{\prime}$ $=\left(\begin{array}{ll}I & 0\end{array}\right)$ or $\left(\begin{array}{ll}I & 0\end{array}\right)^{\prime}$ (depending on whether $j \leqq k$ or $\left.j \geqq k\right)$. The symplectic modular group $\Gamma_{2 n}$ is known (see C. L. Siegel, Math. Ann. vol. 116 (1939) pp. 617-657) to consist of all normal $(n, n)$ arrays. In the present paper it is shown that a given normal $(j, k)$ array can always be completed to an element of $\Gamma_{2 n}$ by placing $(n-j)$ rows after the first $j$ rows, and $(n-k)$ rows after the last $k$ rows. Further, a parmetrization of the general completion of a given normal array is found. This generalizes Siegel's result (loc. cit.) for the special case $j=n, k=0$. (Received July 13, 1953.)

\section{M. F. Ruchte: On measure density: Addition of sets.}

The definitions and notations used are those given by R. C. Buck [Amer. J. Math. vol. 68 (1946) pp. 560-580]. In this paper the sum of two sets of positive integers is studied with respect to measure density. Principal results are: (1) $\underline{\mu}(A)+\bar{\mu}(B)>1$ implies that $A+B \doteq I$ and this result is, in a sense, best possible. (2) $\underline{\mu}(A+B) \geqq \bar{\mu}(A)$ $+\underline{\mu}(B) / 2$ or $A+B=I$ if $1 \in B$. (3) $\mu(A \mp B) \geqq \underline{\mu}(A)+\underline{\mu}(B)$ or $A \mp B \doteq I$, where $A \mp B$ $=A \cup B \cup(A+B)$, providing $\underline{\mu}(A \cap B \cap H)=\overline{0}$ for all $H=[a n]$. The method of the 
proofs is a reduction to the case of finite groups. Definitions of measure density are given for abelian groups, with translates of subgroups of finite index replacing progressions. Using these definitions, the above results are proved for the special case of the Gaussian integers. (Received July 13, 1953.)

588. W. R. Scott: The number of subgroups of given index in nondenumerable Abelian groups.

Let $G$ be an Abelian group of order $A>\aleph_{0}$, and let $\aleph_{0} \leqq B \leqq A$. Then $G$ has $2^{A}$ subgroups of index $B$ and order $A$, and the intersection of all such subgroups is the 0 element. Furthermore, there exist $2^{A}$ subgroups $H_{\alpha}$ of index $B$ and order $A$ such that all the factor groups $G / H_{\alpha}$ are isomorphic to the same direct sum $\sum C_{\beta}$ where either (i) All $C_{\beta}$ are cyclic of prime order, or (ii) all $C_{\beta}$ are $p^{\infty}$ groups where $p$ need not be fixed. The first theorem is an improvement of a result, due to the author (Amer. J. Math. vol. 74 (1952) p. 195), that a nondenumerable Abelian group of order $A$ has $2^{A}$ subgroups of order $A$, and that the intersection of these subgroups is 0 . (Received June 22, 1953.)

\section{Irving Sussman (p) and A. L. Foster: Theory of associate rings. Preliminary report.}

The lattice theory of Boolean rings is a special case of a more general lattice theory holding in a wider classification called associate rings, and which contain as subclasses regular rings without nilpotents, $p$-rings, and algebraic rings in which $a^{n(a)}=a$. Associate rings are subdirect sums of domains of integrity (not necessarily commutative), with identity, with the sole governing restriction that with each element the projected (associated) idempotent is also in the ring. An abstract characterization, independent of the subdirect structure, is given for the commutative case. Via a relation called compatibility ( $a$ is compatible with $b$ if $a^{2} b=a b^{2}$ ) and a generalized partial ordering ( $a$ is enclosed in $b$ if $a b=a^{2}$ ), the ring breaks up into maximal distributive lattices with identities. Under appropriate operative definitions these sets become Boolean rings, all of which are then shown to be isomorphic. A decomposition of the ring into disjoint multiplicative-domains, each determined by an idempotent element, is related to the maximal lattices, and those local multiplicative-domains which are also additively closed and form principal ideals of the ring are identified and related to the component domains of the initial subdirect sum. (Received July 17, 1953.)

\section{G. L. Thompson: Projective quotients in modular lattices.}

In this paper a study is made of quotients of arbitrary (finite) dimension in a modular lattice $L$ in which bounded chains are finite. Given two quotients which are projective in $L$, a generalization of the first normal form for a projectivity, established by R. M. Thrall [Proc. Amer. Math. Soc. vol. 2 (1951) pp. 146-152, \$3], is proved. In this theorem a seven element sub-lattice $\{r ; x, t, u ; w, v, x\}$, such that $\{r ; s, t, u ; w\}$ is a projective root and $x / v$ and $w / t$ are transposes, called a lower root, and its dual called an upper root, play the role of the prime projective root in Thrall's theorem. A root is the lattice generated by a lower or an upper root and a maximal chain between $s$ and $x$, and is characterized as a finite lattice by means of structure numbers. A more complete normal form for a projectivity is given in terms of roots and their structure numbers. [The work on this paper was supported by ONR Contract N8-ONR 71400 at the University of Michigan.] (Received July 9, 1953.) 
591. H. S. Thurston: On matric solutions of a cyclic equation. Preliminary report.

If $f(x)=0$ is a cyclic equation over the rational field, then its companion matrix $A_{1}$ together with $A_{2}=\theta\left(A_{1}\right), A_{3}=\theta\left(A_{2}\right), \cdots, A_{n}=\theta\left(A_{n-1}\right)$ form a conjugate set of rational matric solutions of $f(x)=0$. There exists a rational matrix $T$ which transforms the set $A_{i}$ into a set $B_{i}\left(B_{i}=T^{-1} A_{i} T\right)$ such that $B_{i+1}=U^{-1} B_{i} U$, where $U$ is the matrix obtained by cyclicly permuting the columns of the identity matrix. Thus every cyclic equation over the rational field possesses a conjugate set of rational matrices $B_{i}$ where $B_{i+1}$ is obtained from $B_{i}$ by cyclicly permuting rows and columns. (Received July $17,1953$.

\section{2t. A. D. Wallace: Cohomology, codimension, and mobs.}

To obtain more precise results, not involving a favored coefficient group, it is necessary to use codimension (Haskell Cohen, Tulane Dissertation, 1952). For reasons of brevity we state our results in terms of the cruder covering dimension and employ integral cohomology groups. Let $M$ be a compact connected mob with $\operatorname{dim} M \leqq n$. Let $A$ be a closed subset of $M$ such that $\{x \mid x A \cap A \neq \square\}$ is a proper subset of $M$. If $M$ has a left unit, then $H^{n}(A)=0$. This result is based on some ideas of Deane Montgomery. It follows that, if $M$ has a unit and if $B$ is a closed subgroup of $M$ with $H^{n}(B) \neq 0$, then $B$ coincides with the minimal closed ideal $K$ of $M$. The proof employs Cohen's extension of the Hurewicz theorem to the effect that if $X$ is a compact Hausdorff space of dimension $n$, then $\operatorname{dim}(X \times I)=n+1, I$ being the closed unit interval. It is a corollary that if $e$ is an idempotent of $M$, if $H(e)$ is the maximal subgroup of $M$ including $e$, and if $H^{n}(H(e)) \neq 0$, then $e \in K$. In the corollary we need not assume that $M$ has a unit. If $M$ does not have a unit, then $H^{n}(M) \neq 0$ implies $H^{n}(H(e)) \neq 0$ for each idempotent $e \in K$. (Received January 26,1953.)

\section{3t. F. B. Wright: Ideals in rings of operators.}

Let $A$ be an $A W^{*}$ algebra, and let $L$ be the lattice of projections in $A$. Define a $p$-ideal $P$ of $L$ to be a lattice ideal of $A$ with the property that if $e \in P, f \in L$, and $e \sim f$, then $f \in P$. Then if $I$ is a closed two-sided ideal of $A, I \cap L$ is a $p$-ideal of $L$. If $P$ is a $p$-ideal of $L$, then the right ideal of $A$ generated by $P$ is a two-sided ideal, and the set of projections in the closure of this ideal is precisely $P$. Hence the closed twosided ideals of $A$ are in one-one correspondence with the $p$-ideals of $L$. This is a noncommutative version of Stone's characterization of the closed ideals in $C(X)$. Many results, both known and new, are consequences. Examples: (1) a finite $A W^{*}$ algebra is strongly semi-simple, in the sense of Segal; (2) there is a one-one correspondence between the maximal two-sided ideals of a finite $A W^{*}$ algebra and the maximal ideals of its center; (3) the closed two-sided ideals of an $A W^{*}$ factor form a chain. In particular, in the algebra of all bounded operators on a Hilbert space, the closed two-sided ideals may be identified explicitly. (Received July 17, 1953.)

\section{ANALYsis}

\section{4t. H. A. Antosiewicz and Philip Davis: Some implications of Liapunov's conditions for stability.}

The two fundamental criteria of Liapunov concerning the stability of solutions of systems of differential equations $d x / d t=p(x, t)$, which are based upon the existence of a Liapunov function, are examined. Together with results of I. G. Malkin, K. 
Persidskii, and others they are shown to imply exponential dampedness of all solutions of linear as well as certain nonlinear systems. In particular, it is shown that a necessary and sufficient condition for all solutions of $d x / d t=A(t) x+b(t)$ to be bounded for every bounded vector $b(t)$ is that all solutions of $d y / d t=A(t) y$ are $O\left(e^{-k t}\right)$ for some $k>0$ as $t \rightarrow \infty$. (Received July 20,1953 .)

\section{Richard Arens: Some results of analytic functions generalized.}

Let $Y$ be a compact Hausdorff space and let $A$ be a separating subalgebra with unit of $C(Y$, complex values) with the usual norm. According to Silov, there is a unique minimal closed subset $X$ of $Y$ such that every $f$ in $A$ attains its maximum modulus on $X$. The first proposition regarding $X$ is this: for each point $y$ of $Y$ there is at least one Baire measure $m_{y}$ defined on $X$ such that $f(y)=\int f(x) m_{y}(d x)$ (integration over $X$ ) for every $f$ in $A$. The measure $m_{y}$ is said to represent $y$. When the measure representing $m_{y}$ is unique, the one-parameter family of measures is a generalization of Poisson's kernel. The second proposition is this: with respect to an arbitrary $f_{0}$ in $A$, the family of measures $m_{y}$ may be so selected that $\log \left|f_{0}(y)\right| \leqq \int \log \left|f_{0}(x)\right| m_{y}(d x)$ for that $f_{0}$. Finally, if for any point $y$ and every representing measure $m_{y}$, $\int \log |f(x)| m_{y}(d x)$ diverges to $-\infty$, then $f$ is a topological zero-divisor. (Received July 7, 1953.)

596. Nachman Aronszajn: Boundary values of harmonic functions with finite Dirichlet integral.

A complete characterization of these boundary values for an $n$-dimensional domain $D$ with smooth boundary $B$ (of class $C^{1}$ ) is given. The characterization is obtained by properties of developments in specific orthonormal systems in local coordinates on $B$. In terms of chosen local coordinates on $B$, a quadratic norm is defined for functions on $B$, stronger than the $\dot{L}^{2}$ norm and essentially equivalent to the Dirichlet norm of the corresponding harmonic function. For hypersurfaces $S_{n}$ in $D$ converging to $B$ in a "regular" manner (which implies in particular that the same systems of local coordinates can be used for the $S_{n}$ and for $B$ ) it is proved: if $h$ is a harmonic function in $D$ with a finite Dirichlet integral, the values of $h$ on $S_{n}$ converge strongly in the new norm to the boundary values of $h$. (Received July 17, 1953.)

\section{M. G. Arsove: A generalization of the Dirichlet integral.}

In Euclidean $n$-space let $\Omega$ be a bounded Dirichlet region, $S$ the set of all subharmonic functions bounded on $\Omega$ and taking on continuous values on the boundary $\partial \Omega$, and $A$ the set of all differences of functions in $S$. Under pointwise multiplication the elements $w$ of $A$ form an algebra. If $\Omega^{*}$ is any region with closure in $\Omega$, then $M_{\Omega^{*}}\left(w^{2}\right)$, the total mass of $w^{2}$ on $\Omega^{*}$, and $\int_{\Omega^{*}} w d m$, where $m$ is the mass distribufor $w$, both exist finitely. For $\left\{\Omega_{k}\right\}$ a monotone exhaustion of $\Omega$ let $D(w)$ $=\lim _{k \rightarrow \infty}\left[2 \int_{\Omega_{k}} w d m-M_{\Omega_{k}}\left(w^{2}\right)\right]$. This exists $\geqq 0$ (in the extended sense) independently of the exhaustion and is proportional to the classical Dirichlet integral for functions in $C^{\prime \prime}(\Omega)$. Let $A_{\phi}$ denote the class of all functions in $A$ coinciding on $\partial \Omega$ with a prescribed continuous function $\phi$. If $D(w)$ is finite for some $w \in A_{\phi}$, then $D$ attains a minimum over $A_{\phi}$, the minimizing function being the solution of the Dirichlet problem on $\Omega$ for the boundary function $\phi$. (Received July 20,1953.)

598t. Frederick Bagemihl and Wladimir Seidel: $A$ general principle involving Baire category, with applications to function theory and other fields. 
Let $S$ be a nonempty complete metric space, $P_{n}(s)$ an at most enumerable class of properties meaningful for every $s \in S$, and $Q(s)=\left(P_{1} \bigvee P_{2} \vee \cdots\right)^{\prime}$. Suppose that, for every $P_{n}$, if $X$ is a dense subset of a nonempty open set $G \subseteq S$, and if every $x \in X$ has property $P_{n}$, then every $g \in G$ has property $P_{n}$, and every nonempty open subset of $S$ has an element with property $P_{n}^{\prime}$. Then there exists a residual set $R \subseteq S$, every element of which has property $Q$. This principle leads to a topological analogue of Kronecker's approximation theorem, and results on Hausdorff spaces and functions $f(z)$ meromorphic in $|z|<1$, including the following, some of which contain answers to questions raised by P. Erdös: If the cluster set of $f(z)$ at every point of $|z|=1$ is the whole plane, then that of $f(z)$ on each of a residual set of radii is the whole plane. The conclusion remains valid if, e.g., (1) the set of Fatou points of $f(z)$ on every subarc of $|z|=1$ is of measure less than the length of that subarc; or, on any radius terminating in a point of a set on $|z|=1$, of positive measure on every subarc, (2) $f(z)$ has no limit, or (3) the cluster set of (a nonconstant) $f(z)$ contains a fixed constant. If $E$ is a subset of $|z|=1$, meas $E>0$, and for every $e^{i \theta} \in E$, the cluster set of $f(z)$ on every one of a residual set of segments terminating in $e^{i \theta}$ contains a fixed constant $c$ but not the whole plane, then $f(z) \equiv c$. (Received July 20,1953.)

599. Evelyn Boyd: Regularity preserving factor sequences for Laguerre series.

With the aid of an expression derived by Szegö describing the asymptotic behavior of Laguerre polynomials, it can be shown that the series $\sum_{n=0}^{\infty} f_{n} L_{n}^{(\alpha)}(z)$, where $\alpha>-1$, the $f_{n}$ are arbitrary constants, and the $L_{n}^{(\alpha)}(z)$ are Laguerre polynomials of the complex variable $z$, converges in the interior of a parabola $\operatorname{Re}\left[(-z)^{1 / 2}\right]<\tau$ and possibly on the boundary of this domain. The series defines a function which is analytic at least in the domain of convergence. A sequence $\left\{a_{n}\right\}$ is said to be a regularity preserving factor sequence (r.p.f.s.) if the series $\sum_{n=0}^{\infty} a_{n} f_{n} L_{n}^{(\alpha)}(z)$ converges at least at every point in the domain of convergence of the series $\sum_{n=0}^{n} f_{n} L_{n}^{(\alpha)}(z)$ and if it is possible to continue $F(z)$ analytically along any finite path along which $f(z)$ can be continued analytically. If $g(\omega)$ is defined for $\omega=n(n=0,1,2, \cdots)$ and is analytic in some neighborhood of the point at infinity, then $\{g(n)\}$ is a r.p.f.s. for the series $\sum_{n=0}^{\infty} f_{n} L_{n}^{(\alpha)}(z)$. Likewise, if $G(\omega)$ is an entire function of order $<1 / 2$ and of minimal type if the order equals $1 / 2,\{G(n)\}$ is a r.p.f.s. for $\sum_{n=0}^{\infty} f_{n} L_{n}^{(\alpha)}(z)$. The proofs make use of properties of the differential operator $\delta_{z}=-z D^{2}+(z-\alpha-1) D$. (Received July 10, 1953.)

600. G. U. Brauer: Some Abelian semi-groups of linear transformations of Hausdorff type.

Various semi-groups of Toeplitz matrices of the form $A \mu A^{-1}$, where $A$ is a normal matrix and $\mu$ is a diagonal matrix, are studied with regard to regularity conditions and convergence fields. It is shown that if $m$ is any integer greater than 1 and $C_{m}$ denotes the Cesàro matrix of order $m$, then no regular matrix $C_{m} \mu C_{m}^{-1}$ evaluates divergent sequences. (Received July 22, 1953.)

601. F. E. Browder: Differentiability theorems of the Lichtenstein type for the general linear elliptic differential equation.

Let $K$ be a linear elliptic differential operator of order $2 m$ with suitably differentiable coefficients on a domain $D$ of Euclidean $n$-space. Let $D_{1}$ be a bounded subdomain of $D$ whose closure is contained in $D$. If $e(x, z)$ is a fundamental solution 
for $K$ on $D, \eta \in L^{2}(D)$, then $\phi(z)=\int e(x, z) \eta(x) d x$ is uniquely determined a.e. in $D$ as a limit in square-mean. Generalizing results of Lichtenstein and Friedrichs for the Laplace equation, it is shown that $\phi$ is $2 m$-times differentiable in a certain almosteverywhere sense and that the derivatives obtained satisfy the equation $K \phi=\eta$ a.e. in $D$. (Received July $20,1953$. )

602t. F. E. Browder: The asymptotic distribution of the eigenvalues and eigenfunctions of the general self-adjoint elliptic differential operator. II.

Let $K$ be a self-adjoint linear elliptic differential operator of order $2 m$ with variable coefficients on the bounded domain $D$ of Euclidean $n$-space. The theorems on the asymptotic distribution of the eigenvalues and eigenfunctions of $K$ on $D$ for $2 m>n$, presented by the author in a previous abstract, are established for general $m$ and $n$. For $2 m \leqq n$, mild smoothness conditions on the boundary of $D$ are required in obtaining the eigenvalue distribution but not for the eigenfunctions. Analogous results are obtained for the general self-adjoint strongly elliptic systems of differential equations. (Received July 20, 1953.)

\section{3t. A. P. Calderón and Antoni Zygmund: On a problem of Mihlin.}

Let $x=\left(\xi_{1}, \cdots, \xi_{n}\right), y=\left(\eta_{1}, \cdots, \eta_{n}\right), \cdots$ be points of the $n$-dimensional vector space $E^{n}$ with metric $|x|=\left(\xi_{1}^{2}+\cdots+\xi_{n}^{2}\right)^{1 / 2}$. Let $\Omega(x, \theta)$ be a measurable function of the point $x$ and direction $\theta$ and let $K(x, y)=\Omega(x, \theta)|x-y|^{-n}$, where $\theta$ now denotes the direction from $x$ to $y$, and let $\tilde{f}(x)=\int_{E^{n}} f(y) K(x, y) d y$, with $d y=d \eta_{1}, \cdots, d \eta_{n}$. The following theorem was proved by Mihlin for $n=2$ and stated as conjecture for $n>2$. Consider $\theta$ as a point of the unit sphere $\Sigma$ with center at the origin and suppose that, for all $x, 1^{\circ} \int_{\Sigma} \Omega(x, \theta) d \theta=0,2^{\circ} \int_{\Sigma}|\Omega(x, \theta)|^{2} d \theta<C, C$ a constant independent of $x$ and $d \theta$ the $(n-1)$ st dimensional measure on $\Sigma$. Then the singular integral defining $\tilde{f}(x)$ and taken in the principal value sense converges to a limit in the metric $L^{2}$. Moreover $\|\tilde{f}\|_{2} \leqq A\left\|_{f}\right\|_{2}$. It can be shown that not only is the conjecture correct but that the conclusion holds if the exponent 2 in $2^{\circ}$ is replaced by any $p>2(k-1) / k$, and the latter condition is best possible. (Received September 2, 1953.)

\section{Lamberto Cesari: Frechet surfaces of finite Lebesgue area in $E_{n}$.}

Let $S \subset E_{n}$ be any continuous surface $(n \geqq 3), \quad T: x=T(w), w \in Q, x$ $=\left(x_{1}, x_{2}, \cdots, x_{n}\right)$, any representation of $S(Q$ simple Jordan region of the $w$-plane $\left.E_{2}\right), L(S)$ the Lebesgue area of $S, T_{i j}$ the plane mapping which is the projection of $S$ on the plane $E_{i j}=\left[x_{h}=0, h \neq i, j, h=1,2, \cdots, n\right], E_{i j} \subset E_{n}, W\left(T_{i j}\right)$ the total variation of the plane mapping $T_{i j}, N\left(p ; T_{i j}, Q\right), p \in E_{i j}$, the characteristic function of $T_{i j}$ $(i \neq j, i, j=1,2, \cdots, n), F_{i j}$ the set of all points $p \in E_{i j}$ such that the components of $T_{i j}^{-1}(p)$ are continua of constancy for $T$ on $Q, C$ the boundary curve of $S, r_{i j}$ the $(n-2)$-dim. subspace of $E_{n}$ defined by $\left[x_{i}=0, x_{j}=0\right], R$ the set $R=\sum r_{i j} \subset E_{n}$ where $\sum$ ranges over all $i \neq j, i, j=1,2, \cdots, n$. Let $G_{i j}$ be the set of all points $p \in F_{i j}$ where $N\left(p ; T_{i j}, Q\right)=0$. The following lemma holds (generalization of the analogous lemma for $n=3$; Bull. Amer. Math. Soc. vol. 58 (1952) p. 178): If $C R=0$, if $(0,0)$ $\in G_{i j}$ and is a point of density for $G_{i j}$ in $E_{i j}, i \neq j, i, j=1,2, \cdots, n$, then $C$ is nullhomotop in $E_{n}-R$. The proof is the same as for $n=3$. From here it follows that for every surface $S$, we have $L(S) \leqq \sum W\left(T_{i j}\right)$. This inequality holds also if $Q$ is any finitely connected closed Jordan region, or any open set in $E_{2}$. (Received July 20, 1953.) 


\section{5t. R. B. Davis: $A$ boundary value problem for certain third-order linear composite partial differential equations.}

Using the methods of the author's previous papers, a boundary value problem existence theorem is proved for a certain class of third-order partial differential equations. Uniqueness is not proved, but rather one obtains the Fredholm alternatives. The boundary value problem consists in prescribing $u$ around the boundary of the region, and $\Delta u$ along a suitable curve. The hypothesis is that, besides having a special form, the differential equation has coefficients belonging to $C^{1}$, and the region is simply-connected, with boundary having continuous curvature. The special form is the $(\Delta u)_{x}+a u_{x}+b u_{y}+c u=d$ form described in Proc. Amer. Math. Soc. vol. 3 (1952) pp. 751-756, but with the omission of the terms in $u_{x x}$ and $u_{x y}$, so as to facilitate greatly the estimation of the kernel in the Fredholm integral equation obtained by the method of Levi. (Received July 14, 1953.)

606t. M. R. Demers: On the isoperimetric inequality. Preliminary report.

The isoperimetric inequality is shown to be valid for any subset $A$ of the Euclidean plane having finite Lebesgue (outer) measure if the perimeter of $A$ is taken to be the Favard 1-dimensional measure of the boundary of $A$. Moreover, if equality holds for $A$ and if $A$ has positive Lebesgue (outer) measure, then the interior of the closure of $A$ is a circular disc. It follows immediately from the definitions and a theorem of Federer (Trans. Amer. Math. Soc. vol. 62 (1947) pp. 122 and 182) that the Favard measure may be replaced by Carathéodory linear measure or Gillespie linear measure. (Received July 16, 1953.)

\section{W. F. Donoghue: Invariant subspaces of unitary transforma-} tions.

It is known from the Hellinger-Hahn multiplicity theory that the lattice of closed reducing subspaces of a unitary transformation in a separable Hilbert space is isomorphic to the lattice of sets measurable with respect to a certain Borel measure on the reals modulo the sets of measure zero. Using results due to Wiener, Kolmogoroff, Beurling and Karhunen, the lattice of closed invariant subspaces which are not reducing subspaces (one-sided invariant subspaces) is investigated. (1) The lattice of one-sided invariant subspaces is isomorphic to the lattice of such subspaces for the transformation $V=$ multiplication by $e^{i \theta}$ on the direct sum of $L^{2}(0 \leqq \theta<2 \pi$, Lebesgue measure) with itself $N$ times for some finite or infinite $N$. (2) If $N=1$, the lattice is isomorphic to the multiplicative group $G$ of measurable functions on $0 \leqq \theta<2 \pi$ of absolute value 1 almost everywhere modulo the constants, identifying functions which coincide almost everywhere. If $\phi$ and $\psi$ in $G$ correspond to the subspaces $\mathfrak{M}$ and $\mathfrak{R}$ then $\mathfrak{M} \subseteq \mathfrak{N}$ if and only if $\phi=f \psi$ where $f$ is the boundary value function of a function bounded and analytic in $|z|<1$. (3) Analogous results are obtained for continuous unitary representations of the reals. (Received July 17, 1953.)

608. W. F. Eberlein: Generalized harmonic analysis. I. Preliminary report.

A uniform spectral synthesis for weakly almost periodic functions is obtained under restrictions on the mean rate of growth at infinity. (Received July 20,1953.) 
609. H. W. Ellis (p) and Israel Halperin: Function spaces determined by a levelling length function.

Generalizing the Lebesgue spaces $L^{p}(B)$, the function spaces $L^{\lambda}(B)$ with elements which are functions $f(P)$ valued in a Banach space $B$ and defined on a measure space $S$ with $\|f\|=\lambda(|f(P)|)$ are introduced. Here $\lambda$ is a length function, i.e. for arbitrary measurable, non-negative $u(P): 0 \leqq \lambda(u) \leqq \infty, \lambda(u)=0$ if $u(P)=0$ almost everywhere, $\lambda(u) \leqq \lambda\left(u_{1}\right)$ if $u(P) \leqq u_{1}(P)$ for all $P, \lambda(u+v) \leqq \lambda(u)+\lambda(v), \lambda(k u)=k \lambda(u)$ for $k>0$, and $\lambda\left(\sup u_{n}\right)=\sup \lambda\left(u_{n}\right)$ if the $u_{n}$ are nondecreasing in $n$. The conjugate length function is defined by $\mu(u)=\lambda^{*}(u)=\sup \int u v$ for all $v$ with $\lambda(v) \leqq 1$. A special class called levelling length functions (which include the $L^{p}$ ) are defined by the conditions: $\sup \lambda\left(u_{e}\right)=\lambda(u)$ when $e$ varies over all sets of finite measure and $\lambda\left(u^{\prime}\right) \leqq \lambda(u)$ if $u^{\prime}$ coincides with $u$ except on a set of finite measure and on that set $u^{\prime}$ is constant, equal to the average of $u$ on that set. For levelling length functions: (i) $L^{\lambda}(B)^{*}=L^{\mu}\left(B^{*}\right)$ under conditions less restrictive than Dieudonnés for $L^{p}$ (Canadian Journal of Mathematics vol. 3 (1951) pp. 129-140) and (ii) $L^{\lambda}(V)$ is reflexive under conditions which generalize the corresponding theorem of Phillips for $L^{p}$ (Amer. J. Math. vol. 65 (1943) pp. 108136). (Received July 13, 1953.)

610. Paul Erdös, Fritz Herzog, and George Piranian (p): On Taylor series of functions regular in Gaier discs.

Gaier [Math. Zeit. vol. 56 (1952) pp. 326-334, see p. 327] has shown that if $f(z)$ $=\sum a_{n} z^{n}$ is regular and bounded in a circular disc $|z+a|<1+a(a>0)$, then $a_{n}=O\left(n^{-1 / 2}\right)$. The authors generalize this theorem by showing that if $k$ is a real constant and $(1-z)^{k} f(z)$ is regular and bounded in such a disc, then the Taylor coefficients of $f(z)$ are $O(\phi(n, k))$, where $\phi(n, k)=n^{k-1}, \log n$, or $n^{(k-1) / 2}$, according as $k>1$, $k=1$, or $k<1$. The authors construct functions which show that for each $k$ this bound is the best possible in the sense that the $O$ cannot be replaced by $o$. If $k<0$ then $\sum a_{n} z^{n}$ converges uniformly on the unit circle; and if $k<-1 / 2$ then $\sum\left|a_{n}\right|<\infty$. Fejer's work on the Taylor coefficients of the function $(1-z)^{-k} \exp [1 /(z-1)]$ (see Perron, Arch. d. Math. u. Phys. (3) vol. 22 (1914) pp. 329-340) shows that the latter conclusion does not hold when $k \geqq-1 / 2$ (Received July 17, 1953.)

\section{1t. Robert Finn: On a theorem of S. Bernstein.}

Elliptic partial differential equations which arise from variational problems $\delta \iint F\left(p^{2}+q^{2}\right) d x d y=0, p=\phi_{x}, q=\phi_{y}$, are considered. Conditions on $F\left(\omega^{2}\right)$ are stated which insure that every solution $\phi(x, y)$ regular throughout the $(x, y)$ plane is a linear function $\phi=a x+b y+c$. This theorem was first stated as a property of minimal surfaces by S. Bernstein (Math. Zeit. vol. 26 (1927) pp. 551-558). The precise conditions obtained by the author are rather complicated, but it is sufficient that $F\left(\omega^{2}\right)$ $=\left(1+\omega^{2}\right)^{1 / 2}+\lambda_{\epsilon}\left(\omega^{2}\right)$ where $\epsilon=O\left(\omega^{-1}\right), \epsilon^{\prime}=O\left(\omega^{-3}\right), \epsilon^{\prime \prime}=O\left(\omega^{-5}\right)$ as $\omega \rightarrow \infty$ and $\lambda$ is a real number sufficiently small that $F_{p p} F_{q q}-F_{p q}^{2}>0$. The proof follows in outline that given by T. Rado for minimal surfaces (Math. Zeit. vol. 26 (1927) pp. 559-565). It involves in addition an investigation of certain growth properties of quasi-conformal mappings and the use of the hodograph metric of Chaplygin. The author has knowledge of a similar theorem, proved by other methods, due to L. Bers (Proc. of Arden House Conference, 1952). The author's claim to priority is therefore limited to the methods employed. (Received July 6, 1953.)

612t. Charles Fox: The inversion of convolution transforms by differential operators. 
This paper studies the type of kernel $K(x, v)$ for which the integral equation $f(x)=\int_{0}^{\infty} K(x, v) \phi(v) d v$ is inverted by means of differential operators. Let $E(u)=\prod_{n=1}^{\infty}\left(1+u^{2} a_{n}^{-2}\right)$, where $\sum_{n=1}^{\infty} a_{n}^{-2}$ is convergent and let $K(x, v)$ $=\int_{0}^{\infty}(h(x u) h(v u) / E(u)) d u$, where $h(x)$ is a generalized Fourier kernel (E. C. Titchmarsh, The theory of the Fourier integral, chap. 8). If $y=h(u x)$ satisfies a differential equation of the type $L(x, D) y=-u^{2} y$, where $D$ denotes the differential operator $d / d x$ and $L(x, D)$ is independent of $u$, then $\prod_{n=1}^{\infty}\left\{1-L(x, D) / a_{n}^{2}\right\} f(x)=\phi(x)$. Suitable conditions of convergence are required. An example is $h(x)=x^{1 / 2} J_{\nu}(x)$, where $J_{\nu}$ denotes the Bessel function of order $\nu$. This result generalizes discoveries by Post, Hirschman Jr., and Widder in the theory of inversion of convolution transforms by means of differential operators. (Received June 29, 1953.)

613t. Evelyn Frank and Oskar Perron: Remark on a certain class of continued fractions.

Continued fractions of the form (A) $k_{0} \gamma_{0}+k_{0}\left(1-\gamma_{0} \bar{\gamma}_{0}\right) z / \bar{\gamma}_{0} z-1 / k_{1} \gamma_{1}+k_{1}(1$ $\left.-\gamma_{1} \bar{\gamma}_{1}\right) z / \bar{\gamma}_{1} z-1 / k_{2} \gamma_{2}+\cdots$, where the $k_{v}$ and $\gamma_{v}$ are arbitrary constants different from zero, $\left|\gamma_{v}\right| \neq 1$, were considered by Frank [Proc. Amer. Math. Soc. vol. 3 (1952) pp. 921-937]. To every such continued fraction there corresponds a power series $c_{0}+c_{1} z+c_{2} z^{2}+\cdots$ such that the Taylor's series for the approximants $A_{2 v}(z) / B_{2 v}(z)$, $A_{2 v+1}(z) / B_{2 v+1}(z)$, agree with the power series up to and including the terms involving $z^{v}$ and $z^{v-1}$, respectively. In this paper the function to which (A) converges is considered. It is found that in some cases (A) converges in a larger region than the series, so that (A) represents the analytic continuation. For example, in the case of the geometric series $1+z+z^{2}+\cdots$, depending on the values of the $k_{v}$ and $\gamma_{v}$, (i) (A) may converge over the entire $z$-plane and may equal $1 /(1-z)$, or may equal an entirely different function of $z$; (ii) (A) may converge in various regions to functions which are different. This is the case when $k_{v}=2, \gamma_{v}=(-1)^{v} /(v+2)$; for $|z|<1 / 2$, (A) is equal to $1 /(1-z)$; for $|z|>1 / 2,(\mathrm{~A})$ is equal to $4-1 / z$. (Received July 1,1953 .)

\section{Bernard Friedman and Luna Mishoe (p): A Sturm-Liouville expansion depending on a boundary condition.}

In the classical Sturm-Liouville theory the convergence of the expansion of $f(x)$ in terms of the eigenfunctions of a second-order differential equation does not depend on whether $f(x)$ satisfies the boundary conditions imposed on the differential equation, but depends solely on the local properties of $f(x)$. A case in which the convergence of the expansion to $f(x)$ depends on whether $f(x)$ satisfies a boundary condition is presented. Consider the eigenfunctions of the equation $\left({ }^{*}\right) u^{\prime \prime}+q u+\lambda\left(u^{\prime}+p u\right)$ $=0$, where $u(0)=u(1)=0$. If $f(x)$ is of bounded variation and satisfies the boundary condition $(\dagger) f(0+)+f(1-)$ exp $\left\{\int_{0}^{1} p(t) d t\right\}=0$, then the eigenfunction expansion converges to $\{f(x+0)+f(x-0)\} / 2$. If $f(x)$ is of bounded variation but does not satisfy the boundary condition $(\dagger)$, then the eigenfunction expansion converges to $\{f(x+0)+f(x-0)\} / 2-\left[f(0+)+f(1-) \exp \left\{\int_{0}^{1} p(t) d t\right\}\right] \exp \left\{\int_{0}^{x} p(t) d t\right\}$. This work was performed at Washington Square College of Arts and Science, New York University, and was supported in part by the Office of Scientific Research, H.Q., Air Research and Development Command, U.S.A.F., Baltimore, Md., under Contract No. AF-18(600)-367. (Received July 8, 1953.)

\section{5t. R. E. Fullerton: Properties of $L$ cones in a linear space.}

A cone $C$ in a topological linear space $X$ which is the closure of the convex set determined by its extreme rays is an $L$ cone if for every extreme ray $r$ of $C$ there 
exist $y \in X, y \in C, z \in X$ such that $C \cap(y+C)=z+C$ and $r$ is the ray determined by $z$ and the vertex of $C$. It is shown that a necessary and sufficient condition that $C$ be an $L$ cone is that for any $y \in X$ the set $C \cap(y+C)$ is either a cone or a conoid with a onedimensional finite interval as a vertex. A necessary and sufficient condition that a Banach space $X$ whose unit sphere is determined by its set of extreme points be a space of continuous functions over a compact Hausdorff space is that the cone determined by the origin and a maximal convex subset of the surface of the unit sphere be an $L$ cone which is not a $C$ cone. (Received July 20,1953.)

616t. P. R. Garabedian and M. M. Schiffer: On estimation of electrostatic capacity.

Let $D$ be a domain in space containing the origin, let $S_{r}$ be the sphere of radius $r$ about the origin, let $V_{r}$ be the volume of $D+S_{r}$, and let $\gamma_{r}$ be the capacity constant, with respect to the origin, associated with $D+S_{r}$. By means of Hadamard's variational formula, it is shown that the dimensionless quantity $\gamma_{r}^{3} V_{r}$ is a nonincreasing function of $r$, whence it follows that the quantity has its least value for a sphere about the origin. Let $\Gamma$ be the capacity, with respect to the point at infinity, of a lamina $E$ spanned through a curve $C$. Suppose that the portion of $E$ which was in the region $z>z_{0}$ has been replaced by corresponding sections of the plane $z=z_{0}$, while the rest of $E$, including the boundary curve $C$, remains in the region $z<z_{0}$. Then Hadamard's formula shows that $\Gamma$ is a nondecreasing function of $z_{0}$. The analogous method in two dimensions yields the known theorem that the curve of minimum logarithmic capacity joining two given points is a line segment. Proofs depend upon estimating the the natural charge distribution, which appears in Hadamard's formula, by suitable application of the maximum principle and of comparison domains. (Received June 24, 1953.)

617. Seymour Ginsburg: Order types of simply ordered sets of solutions to problem $\mathrm{P}$.

Let $L$ denote the order type of the real numbers. Theorem: Let $c$ be any order type, of power $2 \boldsymbol{N}_{0}$, which is $<L$. Let $Y$ be any set of power $2 \boldsymbol{N}_{0}$, and $H$ a simply ordered family of subsets of $Y$. Then problem $\mathrm{P}$ (see Trans. Amer. Math. Soc. vol. 74 (1953) p. 514), as applied to $a=c$ and $b=L$, and problem $\mathrm{P}$, as applied to $a=0$ and $b=c$, each admits of a set, which is similar to $H$, of solutions. A consequence of the preceding is the following result: Let $c$ be any order type, of power $2 \mathbf{N}_{0}$, which is $<L$. Then problem $\mathrm{P}$, as applied to $a=0$ and $b=c$, and problem $\mathrm{P}$, as applied to $a=c$ and $b=L$, each admits of a set, of order type $L$, of solutions. Finally, the following generalization of a result due to Sierpinski (Types d'ordre des ensembles linéaires, Fund. Math. vol. 37 (1950) pp. 253-264, Theorem 9) is shown: Let $\left\{a_{m} / m \in M\right\}$, where the power of $M$ is $2 \mathbf{N}_{0}$, be a set of order types, each $a_{m}<L$. Then there exists an order type $c$ such that $a_{m}<c<L$ for each $m$ in $M$. (Received July 13,1953.)

\section{Casper Goffman: On a theorem of Henry Blumberg.}

H. Blumberg proved (Trans. Amer. Math. Soc. vol. 24 (1922) pp. 113-128) the following remarkable, although apparently isolated, fact: Every real function $f(x)$ on $(0,1)$ has an associated dense set $D$ such that $f(x)$ is continuous on $D$ relative to $D$. The purpose here is to show that the analogue for one-one transformations is not valid. (Received July 3, 1953.)

619t. J. W. Green: Further remarks on approximately convex functions. 
Approximately convex functions have been studied by Hyers and Ulam (Proc. Amer. Math. Soc. vol. 3 (1952) pp. 821-828) and the author (Duke Math. J. vol. 19 (1952) pp. 499-504), and it was shown that such a function $f$ can be approximated by a convex function to within $k_{n} \in$ when $k_{n}$ depends on the dimension $n$. The value of $k_{n}$ found is the best possible for $n=1,2,3$. In the present work, more detailed investigation is made into the cases $n=2,3$ of those functions for which the approximation is poorest possible. Typical result: If $F$ is the lower convex envelope of $f$ and $f\left(x_{0}\right)-F\left(x_{0}\right)=k_{n} \epsilon$, then $f-F<k_{n} \in$ near $x_{0}$, and $F$ is linear in precisely a triangle containing $x_{0}$. (Received July 16, 1953.)

620. J. W. Green: Mean values of harmonic functions on homothetic curves.

Let $C$ be a smooth closed curve containing the origin and $C_{\lambda}$ the homothetic transforms of $C$ with respect to the origin and with scale factor $\lambda$. Suppose that, for each harmonic function $U$, the perimeter average of $U$ over all $C_{\lambda}$ is constant, or the area average of $U$ over the interiors of the $C_{\lambda}$ is constant. Then $C$ is a circle. (Received August 21, 1953.)

621. J. G. Herriot: The solution of Cauchy's problem for a third order linear hyperbolic differential equation by means of Riesz integrals.

Cauchy's problem for the wave equation has been solved by means of a generalization of the Riemann-Liouville integral by M. Riesz (Acta Math. vol. 81 (1948) pp. 1-223). In the present paper the explicit solution of Cauchy's problem for the third order partial differential equation $\partial^{3} u /\left(\partial x_{1} \partial x_{2} \partial x_{3}\right)=h\left(x_{1}, x_{2}, x_{3}\right)$ is given by means of a similar generalization of the Riemann-Liouville integral. The discussion is restricted to the case in which $u$ and its first and second derivatives are given on the plane $S$ whose equation is $x_{1}+x_{2}+x_{3}=0$. It is verified in detail that the solution given actually satisfies the differential equation and that it and its derivatives assume the proper values on $S$. The main tool used is the analytic continuation of the integrals which arise. (Received July 3, 1953.)

\section{M. J. Herzberger: Approximate methods in mathematics.}

The calculation with polynomials in which members of higher order are neglected is shown to be equivalent to the calculation with a special system of triangular matrices. It is shown how to apply this fact to obtain an approximate solution of nonlinear partial differential equations and to solve implicit equations. (Received June 10, 1953.)

\section{Edwin Hewitt: A maximum problem in harmonic analyses.}

Let $G$ be a locally compact Abelian group, $\widehat{G}$ its character group, and $p$ a number such that $1<p<2$. Let $f \in \Re_{p}(G)$ and let $\hat{f}$ be the Fourier transform of $f ; \hat{f}$ is a function in $\mathfrak{R}_{p^{\prime}}(\widehat{G})\left(p^{\prime}=p /(p-1)\right)$ such that $\|\hat{f}\|_{p^{\prime}} \leqq\|f\|_{p}$ (Hausdorff's inequality). A subcharacter of $G$ is any function on $G$ which is the product of a complex number, a (continuous) character, and the characteristic function of a compact open subgroup. It is proved that if $G$ is compact or discrete, then equality occurs in Hausdorff's inequality if and only if $f$ is a subcharacter or a translate of a subcharacter. If $G$ is an arbitrary locally compact Abelian group, and if $1 \leqq p \leqq 4 / 3$, then equality occurs in Hausdorff's inequality if and only if $f$ is a subcharacter or a translate of a subcharacter. If $G$ is an arbitrary locally compact Abelian group, if $f$ produces equality in Hausdorff's inequality, and if $f$ is in $\Omega_{p}(g)$ and $\Omega_{p_{1}}(G)$, where $1<p \neq p_{1}<2$, then $f$ is a subcharacter or a translate of a subcharacter. These theorems generalize a 
classical result of Hardy and Littlewood for Fourier series [Math. Ann. vol. 97 (1926) pp. 158-209]. (Received July 9, 1953.)

624. Jack Indritz: Convergence of the Rayleigh-Ritz method to solutions of variational problems. Preliminary report.

Let $R$ be a plane bounded region whose boundary $B$ is a finite number of regular arcs of class $c^{k}, k \geqq 3$, no two intersecting. Let $a, b, c, f$ satisfy $a>0, b>0, c \geqq 0 ; a, b, c$ bounded and integrable, $f$ integrable on $R+B$. Assume that there exists a function $u_{2}^{\prime}$ minimizing the functional $I(u)=\iint_{R}\left(a u_{x}^{2}+b u_{y}^{2}+c u^{2}+2 f u\right) d x d y$ in the admissible set of functions $u$ of class $c^{k}$ on $R+B$ whose values on $B$ are those of a given function $g(x, y)$ of class $c^{k}$ on $R+B$. Here a function is of class $c^{k}$ on $R+B$ if its partial derivatives of order $\leqq k$ exist in $R$ with limits on $B$. Let $G(x, y)$ be of class $c^{k}$ on $R+B$, vanish on $B$, positive in $R$, with positive normal derivative on $B$. The author obtains expansion theorems together with rates of convergence for admissible functions, and finds properties of the expansion analogous to those of Fourier series. An explicit solution is given for $u^{\prime}$ in terms of $g, a, b, c, f$, and functions $f_{i}(x, y)$ obtained by orthonormalizing the set $G x_{2}^{i} y^{j}$ where the square of the norm is $D(u)=\iint_{R}\left(a u_{x}^{2}+b u_{y}^{2}+c u^{2}\right) d x d y$. (Received July 20, 1953.)

\section{Meyer Jerison: The set of all generalized limits of bounded se-} quences.

A construction of Kakutani (Ann. of Math. vol. 42 (1941) p. 1019) is reformulated and extended so as to yield all generalized limits of bounded sequences. Let $M$ denote the Banach space of bounded sequences, $M^{*}$ its conjugate space, $F$ the subset of $M^{*}$ consisting of nontrivial multiplicative functionals, and $T$ the transformation of $M$ into itself which sends $x=\left(\xi_{1}, \xi_{2}, \cdots\right)$ into $T x=\left(\xi_{2}, \xi_{3}, \cdots\right)$. Then every weak*limit point of $\left\{n^{-1} \sum_{i=0}^{n-1} T^{* i f}\right\}_{n=1}^{\infty}$, for each $f \in F$, is a generalized limit, and every generalized limit is in the closed convex hull of those limit points. (Kakutani's construction amounts to considering the functional $f(x)=\xi_{1}$.) This provides an alternative approach to some of the results of G. G. Lorentz (Acta Math. vol. 80 (1948) pp. 167190). (Received July 13, 1953.)

626t. R. V. Kadison: On the orthogonalization of operator representations.

The problem of when a group representation by operators on a Hilbert space is similar to a unitary representation along with the problem of when a representation of a $C^{*}$-algebra is similar to a ${ }^{*}$ representation is investigated. A concept of bounded local semi-simplicity of a group representation is introduced and it is shown that a group representation by operators on a Hilbert space is boundedly locally semisimple if and only if it is similar to a unitary representation. A necessary and sufficient condition is given for a representation of a $C^{*}$-algebra to be similar to a * representation. (Received July 15, 1953.)

627t. Jacob Korevaar: A numerical Tauberian theorem for Lambert series.

The author has recently shown (Best $L_{1}$ approximation and the remainder in Littlewood's theorem, Nederl. Akad. Wetensch. Proc. Ser. A vol. 56=Indagationes Math. vol. 15 (1953)) that the hypotheses $\left|n a_{n}\right|<K_{1}(n=1,2, \cdots)$ and $\left|\sum a_{n} x^{n}-s\right|$ $<K_{2}(1-x)^{\epsilon}(\epsilon>0,0<x<1)$, imply $\left|s_{n}-s\right|<C / \log (n+2), n=0,1, \cdots$. This estimate is best possible. In the present paper an analogous result is proved for Lambert 
series. Let $\mu(k)$ denote the Moebius function and set $\sum_{k \leqq n} \mu(k) / k=g(n)$. Vinogradov has shown that there is a constant $\alpha(1 / 2<\alpha<1)$ for which one can find $A>0, \beta>0$ such that $|g(n)|<A \exp \left\{-\beta(\log n)^{\alpha}\right\}$. From this result and the Tauberian theorem for power series mentioned above the following Tauberian theorem is derived. The hypotheses $\left|n a_{n}\right|<K_{1}$ and $\left|(1-x) \sum a_{n} n x^{n} /\left(1-x^{n}\right)-s\right|<K_{2}(1-x)^{\epsilon}(\epsilon>0,0<x<1)$ imply $\left|s_{n}-s\right|<C /\{\log (n+2)\}^{\alpha}$. If there exists a $\gamma>0$ such that the Riemann zetafunction $\zeta(s)$ has no zeros in the half-plane $\operatorname{Re} s>1-\gamma$, then one may take $\alpha=1$. It is shown by an example that the estimate certainly can not be improved beyond $\left|s_{n}-s\right|<C / \log (n+2)$. (Received July 17,1953 .)

628. Saul Kravetz: On the geometry of Teichmueller manifolds and the structure of the mapping-class group. I. Preliminary report.

Let $\bar{i}(g, h)$ be the Teichmueller manifold of conformal-equivalence classes of topologically-fixed compact Riemann surfaces of genus $g$ with $h$ distinguished points, with the metric induced by extremal quasi-conformal mappings, introduced by $O$. Teichmueller ([1] Abh. der Preuss. Akad. der Wiss., Math. Naturw. Klasse (1939) No. 22; [2] ibid. (1943) No. 4). Let $t \overline{\mathfrak{F}}(g, h)$ be the mapping-class group associated with $t \bar{\Re}(g, h)$. The author solves problems indicated by Teichmueller, proving the following results. $t \bar{\Re}(g, h)$ is an open straight line space [1, $\$ 113-120]$. A necessary and sufficient condition that a certain mapping be conformal is obtained and is applied to proving that the complex lines of $t \bar{\Re}(g, h)$ are hyperbolic planes, by the methods of the metric foundations of geometry $[1, \$ 121]$. The fixed-point set in $t \bar{\Re}(g, h)$ of a finite subgroup of $t \overline{\mathfrak{F}}(g, h)$ is void or a linear subspace of $t \bar{\Re}(g, h)$ homeomorphic to a complex Euclidean space (as indicated by W. Fenchel (Mat. Tidsskr. B. (1950) pp. 90-95), the topological part of this result and Smith's Fixed-Point Theorem solves the Hurwitz-Nielsen realization problem for solvable groups, after noting that it is equivalent to Teichmueller's fixed-point problem, but Fenchel does not mention Teichmueller's contribution $[1, \$ 149])$. (Received May 11, 1953.)

629t. Saul Kravetz: On the geometry of Teichmueller manifolds and the structure of the mapping-class group. II. Preliminary report.

Given any topologically-fixed hyperelliptic surface of genus $g$, its sheet-transformation mapping induces an element of $t \overline{\mathscr{F}}(g, 0)$, which is called a hyperelliptic mapping-class, and the latter leaves fixed a $(2 g-1)$-dimensional linear subspace of $t \bar{\Re}(g, 0)$, which is called a hyperelliptic subspace of $t \bar{\Re}(g, 0)$. The author proves that there are an infinite number of disjoint hyperelliptic subspaces in $t \bar{\Re}(g, 0), g>2[1, \S \S 150-152]$. If $\widetilde{\Re}(g, h)$ is the set of conformal-equivalence-classes of compact Riemann surfaces of genus $g$ with $h$ distinguished points, then $\bar{\Re}(g, h)$ is just $t \bar{\Re}(g, h)$ modulo $t \overline{\mathfrak{F}}(g, h)$. Let $\bar{t} \widetilde{S}(g, h)$ be the set of all points of $t \bar{R}(g, h)$ which are left fixed by elements of $t \overline{\mathscr{F}}(g, h)$ which do not leave all the elements of $t \bar{\Re}(g, h)$ fixed, and let $\bar{S}(g, h)$ be the set of corresponding points on $\bar{R}(g, h)$. It is shown that the mapping-class group of a compact topological surface of genus $g$ with $h$ distinguished points is a discrete topological group. Then $t \overline{\mathfrak{F}}(g, h)$ is shown to be a properly discontinuous group on $t \bar{\Re}(g, h)$. It is then shown that, in the induced topology, $\bar{\Re}(g, h)$ is a Hausdorff space while $\bar{\Re}(g, h)$ $-\widetilde{\Phi}(g, h)$ is a connected topological manifold. $\bar{\Re}(g, h)$ is called the singular moduli manifold. (Received May 11, 1953.)

630. C. E. Langenhop: On a generalization of an inequality and some applications to the boundedness of solutions of differential equations. Preliminary report. 
If $w(t) \geqq 0$ and if for $t \geqq 0$ the inequality $u(t) \lessgtr v(t)+\int_{0}^{t} w(\tau) u(\tau) d \tau$ is true, then $u(t) \lessgtr v(0) \exp \int_{0}^{t} w(\tau) d \tau+\int_{0}^{t} v^{\prime}(\tau)\left(\exp \int_{\tau}^{t} w(s) d s\right) d \tau$. This is a generalization of a well known result when $v(t)$ is a constant. The above inequality can easily be applied to prove such results as the following: let the constant matrix $A$ be such that $\left\|e^{A t}\right\|$ $\leqq K e^{-\sigma_{0} t}, \sigma_{0}>0$, and let the vector function $f(x, t)$ be such that $\|f(x, t)\| \leqq \phi(t)\|x\|$ where $K \phi(t) \leqq \sigma_{0}-\eta, \eta>0$. Then if $p(t)$ is a bounded vector for $t \geqq 0$, all solutions of the equation $d x / d t=A x+f(x, t)+p(t)$ are bounded as $t \rightarrow \infty$ and ultimately satisfy the condition $\|x\| \leqq 2 K \max \|p\| / \eta$. (Received July 20,1953.)

631t. R. B. Leipnik: Von Neumann inverses and negligible sets for semi-gauged operators.

The generalization of the Hahn-Banach Theorem to semi-gauges is used to obtain a simplified proof of the existence of von Neumann inverses for semi-gauged operators. ( $B$ is a von Neumann inverse of $A$ in case $A B A=A$ and $B A B=B$.) The same method is applied to characterize negligible sets for inverses of centered semi-gauged operators. (Received July 16, 1953.)

\section{Mark Lotkin: The propagation of error for methods of integra-} tion.

From both the theoretical as well as practical point of view it is of importance to be aware of the stability properties of numerical methods of integration, and the growth of error in the large. Proceeding along lines similar to those employed by $\mathrm{H}$. Rademacher, H. Rutishauser, L. H. Thomas, and others there have been developed criteria for stability of a number of well known methods of integration of ordinary differential equations. The knowledge of the proper values of the pertinent characteristic equations permits the derivation of expressions for the propagation of error due to truncation and rounding. It is thus possible to obtain satisfactory estimates of the total error accumulated during an integration of a prescribed system of differential equations by means of a particular numerical method. (Received May 12, 1953.)

\section{Lawrence Markus: Asymptotically autonomous differential} systems.

A real, ordinary, first order differential system $S: d x_{i} / d t=f_{i}(x, t), i=1, \cdots, n$, in class $C^{(1)}$ in an open set of Euclidean $n$-space for all $t>t_{0}$, is asymptotic to the autonomous system $\mathcal{S}_{\infty}: d x_{i} / d t=f_{i}(x)$ in case $\lim _{t-\infty} f_{i}(x, t)=f_{i}(x)$, where the convergence is uniform on compact sets. By using the intermediate autonomous systems $S_{t^{*}}: d x_{i} / d t$ $=f_{i}\left(x, t^{*}\right)$, for each fixed $t^{*}$, and the limit system $S_{\infty}$, one investigates the boundedness, periodicity, and other asymptotic properties of the solutions of $S$. In particular one generalizes the plane Poincaré-Bendixson theory. (Received July 15, 1953.)

\section{4t. F. I. Mautner: Ergodic double cosets.}

Let $G$ be a locally compact topological group and $H$ a closed subgroup such that the coset space $G / H$ has an invariant measure whose total measure is finite. Assume also that $G$ has no nonconstant finite-dimensional continuous unitary representations. Then the double cosets of $H$ in $G$ are ergodic in the sense that any measurable function $f(g)$ on $G$ which satisfies (almost everywhere) $f\left(h g h^{\prime}\right)=f(g)$ for $h, h^{\prime} \in H, g \in G$ is a constant. Examples: Let $G$ be the group of $2 \times 2$ real matrices of determinant one and $H$ the modular group, or for example $H$ can be the fundamental group of any 
(compact) Riemann surface (of genus $>1$ ). More generally, let $G$ be any of the noncompact classical (simple Lie) groups and $H$ one of the discontinuous subgroups studied by C. L. Siegel (Symplectic geometry, Amer. J. Math. vol. 65 (1943) pp. 1-86). The above should be contrasted with a result of C. L. Siegel (Discontinuous groups, Ann. of Math. vol. 44 (1943) pp. 674-689) according to which under other conditions the double sets behave discontinuously, i.e. in the opposite manner from the above. (Received May 11, 1953.)

\section{5t. F. I. Mautner: Note on the Fourier inversion formula on} groups.

Properties of the generalized Peter-Weyl-Fourier transform on locally compact groups are studied in more detail. Among other things it is shown that this generalized Fourier transform maps the space $L_{2}(G)$ of square integrable functions on $G$ onto a certain space of operator valued functions. This latter space can be described independently as a certain space of measurable operator valued functions. From this one obtains easily various formulations of the generalized Fourier-inversion formula. These results are needed to study the Fourier transform of more general objects than functions such as distributions on groups. This will be carried out in detail in another publication. (Received May 4, 1953.)

\section{6t. Zeev Nehari: On the zeros of solutions of second-order linear dif- ferential equations.}

This paper is concerned with the relations between the function $p(z)$ and the location of the zeros of the solutions of the differential equation $w^{\prime \prime}(z)+p(z) w(z)=0$, both in the case in which $p(z)$ is an analytic function of the complex variable $z$ in the half-strip $\operatorname{Re}\{z\} \geqq 0,-1<\operatorname{Im}\{z\}<1$, and in that in which $p(z)$ is a continuous function of $z$ for $z \geqq 0$. Example of the results obtained: If $\int_{0}^{\infty} x|p[x \pm i(1-\delta) y]| d x<\infty$ $(z=x+i y)$ for sufficiently small $\delta$ and $|p(x+i y)|=o(x)$ for large $x$ and $-1<y<1$, then no solution of the equation can have an infinite number of zeros in the halfstrip $x \geqq 0,-1+\epsilon<y<1-\epsilon(0<\epsilon<1)$. (Received September 2, 1953.)

\section{Emanuel Parzen: Some conditions for uniform convergence of integrals.}

Let $m$ be a measure on a space $R$, and for each $t \in T$ and $n=1,2, \cdots$, let $f_{n}(x, t)$, $f(x, t)$ be functions measurable in $x \in R$. It is of some interest to determine conditions for statements of the form (1) $\int_{R}\left|f_{n}(x, t)-f(x, t)\right| d m \rightarrow 0$ uniformly in $t$ as $n \rightarrow \infty$. In this paper, we define modes of uniform convergence of a family of sequences of measurable functions which permit us to obtain necessary and sufficient conditions for (1) to hold. The main notions we define are (I) $f_{n}(x, t) \rightarrow f(x, t)$ almost everywhere $m$-uniformly in $t$ if, for every $\epsilon>0$ and every measurable set $A$ of finite measure, as $N \rightarrow \infty, m\left\{x \in A:\left|f_{n}(x, t)-f(x, t)\right|>\epsilon\right.$ for some $\left.n>N\right\} \rightarrow 0$ uniformly in $t$, and (II) $f_{n}(x, t) \rightarrow f(x, t)$ in measure $m$-uniformly in $t$ if, for every $\epsilon>0$, as $n \rightarrow \infty, m\left\{x: \mid f_{n}(x, t)\right.$ $\left.-f(x, t) \mid>_{\epsilon}\right\} \rightarrow 0$ uniformly in $t$. Most of the relations that hold in measure theory between convergence almost everywhere, convergence in measure, and convergence in mean continue to hold between (I), (II), and (1). (Received July 17, 1953.)

\section{Pasquale Porcelli: Concerning R. E. Lane's integral.}

If $g$ is a bounded and nondecreasing function on the set of all numbers, then the outer $g$-length $l_{g} S$ of the number set $S$ is the largest number $k$ such that $k$ 
$\leqq \sum_{(G)}[g(q-)-g(p+)]$ for every countable collection $G$ of segments $(p, q)$ covering $S$. Theorem. If $\epsilon>0, \delta>0$ and $l_{g} S>\delta$, then there exists a nondecreasing simple stepfunction $h$, such that $|h(x)-g(x)|<\epsilon$ for every number $x$ and $l_{h} S>\delta$. By means of this theorem it is shown that if $f$ is $g$-summable on the interval $[a, b]$, i.e., if $f$ is the limit on $[a, b]-T, l_{g} T=0$, of a uniformly bounded sequence $\left\{h_{n}\right\}_{n=1}^{\infty}$ of simple stepfunctions and $h_{n}(x+) \rightarrow f(x+)$ if $g(x+)>g(x)$ and $h_{n}(x-) \rightarrow f(x-)$ if $g(x)>g(x-)$, then the sequence $\left\{\int_{a}^{b} h_{n} d g\right\}_{n=1}^{\infty}$ of Lane integrals converges to a limit $\int_{a}^{b} f d g$ depending only on $f$. The class of $g$-summable functions contains Lane's $g$-integrable functions, and Riemann integrable and Lebesgue summable functions $(g(x) \equiv x$ on $[a, b])$. Integration by parts is retained in case $h_{n}(x) \rightarrow f(x)$ everywhere in $[a, b]$. (Received May 27, 1953.)

\section{9t. M. H. Protter: A singular initial value problem for a class of} hyperbolic equations.

The initial value problem for equations of the form $K(z)\left(u_{x x}+u_{y y}\right)-u_{z z}+a(z) u_{z}=0$ is treated. The function $K(z)$ is a positive nondecreasing, continuously differentiable function, and the case $K(0)=0$ is allowed; $a(z)$ is a positive smooth function which is $O\left(1 / z^{\alpha}\right), z \rightarrow 0, \alpha \leqq 1$. Under these conditions the Cauchy problem is solved with initial data on the parabolic plane, $z=0$. The method used is a generalization of the method used by M. H. Martin for the wave equation. For $K(z) \equiv 1, a(z)=k / z$, this yields the solution of the Cauchy problem for the Euler-Darboux equation, while the case $a(z) \equiv 0, K(0)=0, K^{\prime}(0) \geqq 0$ extends the results obtained by Bers for the equation $K(y) u_{x x}-u_{y y}=0$. (Received March 30, 1953.)

\section{L. T. Ratner: On approximately convex sets.}

D. H. Hyers and S. M. Ulam [Proc. Amer. Math. Soc. vol. 3 (1952) pp. 821-828] and J. W. Green [Duke Math. J. vol. 19 (1952) pp. 499-504] have considered the concept of approximately convex function. (A real-valued function $f(x)$ defined on a convex subset $S$ of $E_{n}$, the $n$-dimensional Euclidean space, is said to be $\epsilon$-convex provided that $f(h x+(1-h) y) \leqq h f(x)+(1-h) f(y)+\epsilon$ for all $x$ and $y$ in $S$ and for every $h, 0 \leqq h \leqq 1$.) Let $M$ be a closed and bounded subset of $E_{n} ; f(x)=d(x, M)$ is a convex function on the whole space if and only if $M$ is a convex set, as is well known. In the present paper the concept of approximately convex sets is linked to that of approximately convex functions; $M$ is said to be $\epsilon$-convex provided that $f(x)$ is $\epsilon$-convex. Then $d(M, H M) \leqq k \epsilon$ (where $H M$ is the convex hull of $M$, the distance is the Hausdorff distance between $M$ and $H M$, and $k$ depends upon the dimension of $E_{n}$ ). Conversely, if $d(M, H M) \leqq \epsilon$ then $f(x)$ is $\epsilon$-convex. Examples are given showing the necessity of the constant $k$. Approximately convex sets which also possess other properties of interest are characterized. (Received July 16, 1953.)

\section{1t. R. M. Redheffer: A theorem of Carlson.}

Let $F(z)=\Pi\left(1-z^{2} / \lambda_{n}^{2}\right), \lambda_{n}>0, \Lambda(u)=$ number $\lambda$ 's $\leqq u$. With $p(v, x)=\Lambda(v x) / v x$ $-\Lambda(x / v) /(x / v)$, condition A is $\lim _{\delta \rightarrow 0} \lim _{x \rightarrow \infty} \int_{\delta}^{1-\delta} p(v, x) d v /\left(1-v^{2}\right)=0$. Previously it was shown that A implies $\log ^{+}|F(x)|=o(x)$ if $\Lambda(u) / u<M$. Now it is shown that $\log ^{+}|F(x)|=o(x)$ implies A if $\lambda_{n+1}-\lambda_{n}>c>0$. The first result generalizes, and the second completes, a theorem of Carlson that $\log ^{+}|F(x)|=o(x)$ if $\lambda_{n+1}-\lambda_{n}>c$ and $\lim \Lambda(u) / u$ exists. (Received February 12, 1953.)

642t. R. M. Redheffer: Entire functions with uniformly distributed zeros. 
Let $F(x)=\Pi\left(1-x^{2} / \lambda_{n}^{2}\right)$ where $\lambda_{1} \leqq \lambda_{2} \leqq \lambda_{3} \cdots$ and let $\Lambda(u)$ be the number of $\lambda$ 's $\leqq u$. Suppose $|\Lambda(u)-D u| \leqq H$ for some constants $D>0, H>1 / 2$. Paley and Wiener showed $|F(x)|<C|x|^{4 H-1}$ for some constant $C$. The author later showed $|F(x)|$ $<C|x|^{4 H-2}$, and gave a counterexample for each $x$. If $F(x)$ is fixed, it is now proved that $F(x)=o\left(|x|^{4 H-2}\right)$. Moreover, let $C(x) \rightarrow 0$. Then there is an $F(x)$ such that $F(x)>C(x) x^{4 H-2}$ for an infinite sequence $x=x_{i} \rightarrow \infty$. The proof makes essential use of the fact that $\Lambda(u)$ is an integer. Let $|\Lambda(u)-D u| \leqq H(u)$ where $H^{\prime \prime}(u) \leqq 0, \lim H(u)$ $=\infty, \lim H^{\prime}(u)=0$. Then $\log |F(x)| \leqq G(x)$ where $G(x) \sim 4 H(x) \log [x / H(x)]$. Moreover, there is a fixed function $F(x)$ and a sequence $x=x_{i} \rightarrow \infty$ such that log $|F(x)|$ $>G_{1}(x)$ where $G_{1}(x) \sim 2 H(x) \log [x / H(x)]$. Finally, there is a function $H(u)$ of the above type for which the first result is best possible, and also a function for which the second is best possible. (Received February 19, 1953.)

\section{R. M. Redheffer: Sets of exponentials with finite deficiency.}

According to Paley and Wiener, the set $\left\{e^{ \pm i \lambda_{n x}}\right\}$ has deficiency $D$ if it becomes complete on a given interval when $D$ but not when fewer functions $\left\{e^{i \alpha x}\right\}$ are adjoined to the set. If $D=0$ the set is exact. If $\lambda_{n}$ is periodic, in the sense that $\lambda_{n+p}$ $=\lambda_{n}+A$ for some fixed $A$ and $p$, and if $\lim n / \lambda_{n}<\infty$, then $\left\{e^{i \lambda_{n} x}\right\}$ is exact on a finite interval. This results from the following generalization of a theorem of Paley and Wiener: Let $\sum 1 /\left|\lambda_{n}\right|^{2}<\infty$, let $F(z)=\prod\left(1-z^{2} / \lambda_{n}^{2}\right)$, and let $\left\{e^{ \pm i \lambda_{n} x}\right\}$ have finite (positive or negative) deficiency. Then the deficiency is $D$ if and only if $\int_{1}^{\infty} x^{2 D-2}|F(x)|^{2}$ $<\infty, \int_{1}^{\infty} x^{2 D}|F(x)|^{2} d x=\infty$. There exists a set $\lambda_{n}$ and a set $\lambda_{n}{ }^{\prime}$ such that $\left|\lambda_{n}-n\right|<2$, $\left|\lambda_{n}{ }^{\prime}-n\right|<2, \lambda_{n}<\lambda_{n}{ }^{\prime},\left\{e^{ \pm i \lambda_{n x}}\right\}$ is complete, and $\left\{e^{ \pm i \lambda_{n}^{\prime} x}\right\}$ is not complete. One can even have $\lambda_{n}<\lambda_{n-1}^{\prime}$. One can construct sets exact on intervals $D$ and $D^{\prime}$ such that the combined set has arbitrarily large deficiency on $D+D^{\prime}$. (Received February 12, 1953.)

644. M. S. Robertson: Schlicht solutions of the Sturm-Liouville equation.

Let $z^{2} q(z)=\sum q_{n} z^{n}$ be nonconstant and regular in $|z|<1, q_{n}$ real, $q_{0} \leqq 1 / 4$. Let $\Re z^{2} q(z)$ assume its maximum on $|z|=r$, for every $r<1$ at $z=r$. A unique universal constant $A=A(q)$ associated with $q(z)$ is determined so that the unique solution $W_{A}(z)=\sum \beta_{n} z^{n+\beta}, \beta_{0}=1$, of $W^{\prime \prime}+\left(A\left(q(z)-q_{0} z^{-2}\right)+q_{0} z^{-2}\right) W=0$, corresponding to the larger root $\beta$ of the indicial equation, is such that, when raised to the power $1 / \beta$, it is schlicht and star-like in $|z|<1$, the radius of univalency being one when $0<A<\infty$. For constants $C>A$ the corresponding solutions are not schlicht in $|z|<1$. Let $z^{2} p(z)$ be regular in $|z|<1, \gamma$ real, $|\gamma| \leqq \pi / 2$, for which, in $|z|<1, \Re\left(e^{i \gamma} z^{2} p(z)\right)$ $\leqq \cos \gamma\left(A\left(|z|{ }^{2} q(|z|)-q_{0}\right)+q_{0}\right), A=A(q)$. Let $W(z)=\sum a_{n} z^{n+\alpha}, a_{0}=1$, be the unique solution of $W^{\prime \prime}+p(z) W=0$, corresponding to the root $\alpha$ of the indicial equation with the larger real part. Then $W(z)$, raised to the power $1 / \alpha$, is schlicht and spirallike in $|z|<1$. The constant $A(q)$, constructively determined, is the largest possible one for a given $q(z)$. (Received July 10, 1953.)

\section{5t. Walter Rudin: A theorem on maximum modulus algebras.}

Let $K$ be the closure of a plane domain $D$ whose boundary $C$ consists of $n$ simple closed nonintersecting curves. Let $A$ be an algebra of complex-valued functions $f$, continuous on $K$, such that $|f(z)|$ attains its maximum on $C$. Theorem I: If $A$ contains all functions analytic and single-valued on $K$, then every member of $A$ is analytic in $D$. This extends a result previously obtained for simply connected domains (Bull. Amer. Math. Soc. vol. 58 (1952) p. 646). The proof depends on the repre- 
sentation of linear functionals as measures, and on Theorem II: Suppose $C$ consists of $n$ analytic curves. If $\mu$ is a complex measure on $C$ such that $\int_{C} g(t) d \mu(t)=0$ whenever $g$ is analytic and single-valued on $K$, then $d \mu(t)=f(t) d t$, where $f \in L_{1}$ on $C$; the function $F(z)=(1 / 2 \pi i) \int_{C} d \mu(t) /(t-z)$, which is analytic in $D$, has nontangential boundary values $f$ almost everywhere on $C$. Theorem II is proved by induction on $n$. (Received May 7, 1953.)

\section{Charles Saltzer: Discrete potential theory in two dimensions. Preliminary report.}

The results below are based upon the finite difference analogues of Green's first and second identities given by Courant, Friedrichs, and Lewy (Math. Ann. (1928)), and the fundamental solution of the five point Laplace difference equation given by McCrea and Whipple (Proc. Roy. Soc. Edinburgh (1939)), and Stöhr (Math. Nach. (1950)). In this paper a theory of discrete harmonic functions, i.e. functions satisfying the Laplace difference equation, is developed within the framework of the difference equation which parallels classical potential theory. The analogues of Green's third identity, double and simple layer potentials as well as an analogue of the discontinuity of a double layer potential at the boundary are given. The last result is used to reduce the boundary value problems of the first and second kind to finite difference analogues of the integral equations of potential theory. The associated NeumannPoincaré series are shown to converge for all regions, finite, infinite, simply or multiply connected provided the boundaries are finite. Regularity at infinity is defined and multiple-valued solutions are given. Generalizations and applications are indicated and existence and uniqueness theorems are given. (Received September 3,1953.)

647. Francis Scheid: Difference methods and the initial value problem for a parabolic equation of arbitrary even order.

In this paper it is proved that the differential equation $u_{t}=\sum_{0}^{2 p} a_{n}(x, t) u^{(2 p-n)}$ $+d(x, t)$ has a solution for $t \geqq 0,-\infty<x<\infty$, satisfying $u(x, 0)=f(x)$, provided $f(x)$, $d(x, t)$, and all $a_{n}(x, t)$ are of class $C^{4 p}$ (uniformly continuous and bounded together with their first $4 p$ derivatives with respect to $x$ ) and their first time derivatives are $C^{0}$, and where $a_{0}=(-1)^{p} A_{0}$ with $A_{0} \geqq \delta>0$. The solution is of class $C^{2 p}$. A uniqueness theorem is also obtained. Fritz John has treated in a fundamental paper (Comm. on Pure and Appl. Math., May, 1952) the case $p=1$. He obtained the solution as the limit of a convergent difference process. His methods prove to be adequate for the general case and are closely followed. Using the result here obtained a reduction of hypothesis will be affected in a second paper. (Received May 14, 1953.)

648t. J. T. Schwartz: Analytic perturbations of operators in Banach spaces.

Theorem: Let $A(z)$ be an analytic function of the complex variable $z$ defined for $z$ in a domain $D$ and having values in the algebra $B(X)$ of bounded operators on a Banach space $X$. Suppose that $0 \in D$, and that $A(0)$ has an eigenvalue $\lambda$ of multiplicity $m$ such that some circle $C_{\delta}=\{\mu|| \lambda-\mu \mid<\delta\}$ contains no points of $\sigma(A(0))$ other than $\lambda$. Then there exists an $\epsilon>0$ such that for $|z|<\epsilon, \sigma(A(z)) \cap C_{\delta}$ consists of a finite number of points $\lambda_{1}(z) \cdots \lambda_{k}(z), k \leqq m$. Each $\lambda_{i}(z)$ can be expressed as a fractional power series in $z$, and each corresponding projection $E_{i}(z)$ as a fractional Laurent series in $z$ with a singularity of finite order at $z=0$. Corollary: If $A(z)$ is normal for all real $z \in D$, then $\lambda_{i}(z)$ and $E_{i}(z)$ depend analytically on $z$. The proof will appear in a forthcoming book on spectral theory. (Received May 15, 1953.) 


\section{9t. V. L. Shapiro: Uniqueness of double trigonometric integrals.}

Let $c(u, v)$ in $L^{2}$ on any bounded domain be $o\left(\left(u^{2}+v^{2}\right)^{-\epsilon}\right), \epsilon>0$. Suppose the double trigonometric integral $\int_{E_{2}} e^{i(x u+y v)} c(u, v) d u d v$ is $(C, 1)$ circularly summable to $f(x, y)$. If $f$ satisfies a Lipschitz condition of order $\alpha$ on every bounded domain ( $\alpha$ depending on the domain), it is then shown in this paper that $\left(4 \pi^{2}\right)^{-1} \int_{E_{2}} e^{-i(x u+y v)} f(x, y) d x d y$ is $(C, 1)$ circularly summable to $c(u, v)$ almost everywhere. Using the fact that the integral is equisummable with a trigonometric series, the proof is brought about by showing that the generalized Laplacian of the Riemann function associated with the integral is equal to $f$. (Received June 22, 1953.)

\section{A. L. Shields: Hausdorff dimension of a vector sum. Preliminary} report.

Let $A$ and $B$ be two sets of real numbers. Then their vector sum, $A+B$, is the set of all numbers $a+b, a$ in $A, b$ in $B$. Let $\mathrm{Hd} A$ denote the Hausdorff dimension of $A$. Salem conjectured (1) $\mathrm{Hd}(A+B) \leqq \mathrm{Hd} A+\mathrm{Hd} B$. This is unproven. In an attempt to prove it we are led to the following. Let $m$ be a non-negative, totally finite measure on the Borel sets on the line. Let $a(m)=\sup \alpha$ such that $\iint\left(1 /|x-y|^{\alpha}\right) d m(x) d m(y)$ $<\infty$. Then the following two statements would imply Salem's conjecture: (2) $a(m * n) \leqq a(m)+a(n)$, where $m * n$ denotes convolution; (3) given a measure 1 on $A+B$ (i.e. $A+B$ complement is a null-set for 1 ), then there exist measures $m$ and $n$ on $A$ and $B$ respectively, such that $(m * n)(E) \leqq 1(E)$ for every Borel set $E$. (2) is proven for the special case when $m$ and $n$ are the same. (Received July 20,1953.)

651t. Maurice Sion: On the existence of functions having given partial derivatives on a curve.

Let $m$ and $n$ be positive integers. Let $A \subset E_{n}$ be a homeomorphic image of the unit interval. If functions on $A$, satisfying certain conditions depending on $m$ and suggested by Taylor's formula, are given, then a sufficient condition on $A$ is found for the existence of a function $f$ of class $C^{(m)}$ on $E_{n}$ whose partial derivatives through order $m$ agree with the given functions on $A$. The condition depends on $m$ in such a way that as $m$ increases more curves satisfy it. For any $m$ there are curves that satisfy this condition and yet possess infinite Carathéodory $(m-1)$-measure. If $m=1$, the condition reduces to having the ratio of the length of arc to chord between any two points uniformly bounded. These results are applied to certain curves and functions originally defined by $\mathrm{H}$. Whitney to show that if $m$ and $n$ are integers, $n \geqq 2$, then there exists a function $f$ of class $C^{(n+m-1)}$ on $E_{n}$ and a homeomorphic image $P$ of the unit interval, $P \subset E_{n}$, such that: all the partials of $f$ of order greater than $m$ vanish everywhere on $P$; all the partials of $f$ vanish at one point of $P$; and yet $f$ is not constant on $P$. (Received July 13, 1953.)

\section{K. T. Smith: Positive functions in spaces with reproducing kernel.}

The natural order determined by the positive functions in a functional class with reproducing kernel is discussed. The results obtained relate positivity of the kernel to properties of the functional class. Applications to Green's functions of second order elliptic partial differential equations are given. (Received July 17, 1953.)

653. H. H. Snyder: Monogenic functions and residue theory in a harmonic algebra. 
The product $(1, i) \times(1, j)$ of the ordinary complex algebra with itself is a linear associative commutative algebra over the real field $(1, i, j, k)$. Multiplication is defined by $i^{2}=j^{2}=-1=-k^{2}, i j=k, i k=-j, j k=-i$. Let $w=x+i a y+j b z+k u$ (where $a^{2}+b^{2}=1$ ) denote the variable whose range is the algebra. Developing ideas of P. W. Ketchum (Trans. Amer. Math. Soc. vol. 30), monogenic functions $f(w)=U_{1}$ $+i U_{2}+j U_{3}+k U_{4}$, where the $U_{k}$ are real-valued functions of the real variables $x, y, z, u$, are defined. A set of linear first order partial differential equations in the $U_{k}$ which characterize monogenicity of $f(w)$ are obtained. Theorem: Let $u$ be fixed but arbitrary, and let $R$ be a region of three-space in which $f(w)$ is monogenic. Then $\nabla^{2} U_{k}=0$ throughout $R$ (where $\left.\nabla^{2}()=()_{x x}+()_{y y}+()_{z z}\right)$. Elementary (monogenic) functions of $w$ are defined. Two analogues of the Euler polar representation are obtained. Singularities of functions (which have them) are found to consist of two lines through the origin. Line and surface integration are defined and generalizations of the Cauchy integral formulas obtained. The residue of a function at a singular line is defined, and applications are made to the evaluation of real line integrals in three dimensions. Geometrical properties are found which relate this class of harmonic functions to the real and imaginary parts of an ordinary analytic function $f(x+i y)$. (Received July $17,1953$. )

\section{D. L. Thomsen, Jr.: On an integral containing one small and} two large parameters.

The integral $J=\int_{0}^{a} \exp [-h \phi(t)+k \psi(t)] d t(a>0)$ is considered as $h, k \rightarrow+\infty$ and $a \rightarrow 0^{+}$. The functions $\phi(t)$ and $\psi(t)$ satisfy suitable continuity conditions, and in addition it is assumed $\phi(0)=\phi^{\prime}(0)=\psi(0)=0, \phi^{\prime \prime}(0)>0$, and $k=o(h)$. Then considering the various rates with which $a \rightarrow 0^{+}$, with which $h, k \rightarrow+\infty$, and the sign of $\psi^{\prime}(0)$, the integral $J$ is shown to be asymptotic to suitable forms of the error function. (Received July 17, 1953.)

\section{H. L. Turrittin: Convergent solutions for linear differential equations with analytic coefficients.}

The author has shown in the most general case that the divergent asymptotic series solutions representing a fundamental set of solutions corresponding to a singular point of a system of linear homogeneous differential equations with analytic coefficients can be summed and the divergent series solutions replaced by convergent generalized factorial series, thus completing the work of W. J. Trjitzinsky, Trans. Amer. Math. Soc. vol. 37 (1935) pp. 80-119. First the linear matrix differential equation is reduced to a suitable canonical form, an extension of that given by M. Hukuhara, J. Fac. Sci. Hokkaido Imp. Univ. (I) vol. 5 (1937) pp. 123-166. The analysis then proceeds in steps paralleling those given by Trjitzinsky, the computations always being carried out in matrix notation to minimize the algebraic complications; and convergence of the factorial series is finally proved by estimating the rate of growth of solutions of a related system of integral equations. For example Trjitzinsky's problem, p. 118, is easily handled by a change of independent variable $x=z^{2}$, whereupon it is found that his equation has a convergent generalized factorial series solution of the form $1+\sum_{s=0}^{\infty} a_{s} /\left[x^{1 / 2}\left(x^{1 / 2}-\gamma\right)\left(x^{1 / 2}-2 \gamma\right) \cdots\left(x^{1 / 2}-s \gamma\right)\right]$ where the $a$ 's and $\gamma$ are suitably chosen constants and $x$ is limited to the proper curvilinear sector extending to infinity in the complex $x$-plane. (Received July 14, 1953.)

656. J. G. Wendel: On uniqueness of Fourier-Stieltjes transforms. $G=\{g\}$ is a locally compact abelian group, $G^{*}=\left\{g^{*}\right\}$ its character group, $L=\{x\}$ 
its Banach algebra of complex-valued integrable functions, $M$ its algebra of measures $m$ of bounded variation $\|m\|$; as usual, $L$ is identified with the subset of absolutely continuous $m \in M$. The well known fact that $m$ is uniquely determined by its Fourier-Stieltjes transform $m\left(g^{*}\right)=\int g^{*}(g) m(d g)$ is here expressed algebraically as follows. Let $M$ be endowed with any topology in which it becomes a topological algebra containing $L$ as a dense subset, and in which the functions $m\left(g^{*}\right)$ are continuous. Then the closed maximal ideals of $M$ are precisely the sets $M\left(g^{*}\right) \equiv\left\{m \mid m\left(g^{*}\right)=0\right\}$. One such topology is: $m_{\alpha} \rightarrow m$ means $\left\|x m_{\alpha}-x m\right\| \rightarrow 0, x \in L$, and $\sup _{\alpha}\left\|m_{\alpha}\right\|<\infty$, where for $x \in L, m \in M$ we define $x m \in L$ by $(x m)(g)=\int x(g-h) m(d h)$. (Šreider [Mat. Sbornik vol. 27 (69) (1950) pp. 297-318)] viewed $M$ as a Banach algebra and showed that it has a profusion of additional maximal ideals (all necessarily closed in the norm topology), so many more that the $M\left(g^{*}\right)$ 's are neither dense in, nor the boundary of, the space of all maximal ideals. ) (Received July 24, 1953.)

\section{F. B. Wright: $A$ class of factors of type $\mathrm{II}_{1}$.}

Let $A$ be an $A W^{*}$ algebra (Kaplansky, Ann. of Math. vol. 53 (1951) pp. 235-249) of finite class, and suppose that $A$ has a trace defined in it. Let $M$ be any maximal two-sided ideal of $A$. If $A$ is of type $\mathrm{II}_{1}$, then $A / M$ is an $A W^{*}$ factor of type $\mathrm{II}_{1}$ for every $M$. In case $A$ is of type I (in which case $A$ necessarily has a trace), then $A / M$ is an $A W^{*}$ factor of type $\mathrm{I}(n)$ for all $M$ except possibly for a closed nowhere-dense set in the space of maximal two-sided ideals of $A$. This set is empty if and only if $A$ is the direct sum of a finite number of homogeneous algebras of type I. In case this set is not empty, then $A / M$ is an $A W^{*}$ factor of type $\mathrm{II}_{1}$ for every $M$ in the set. This shows the existence of a class of $A W^{*}$ factors which are (1) not a priori weakly closed, and (2) not a priori related to the few previous known examples of such factors. (Received July $17,1953$. )

\section{A. D. Ziebur: On a double eigenvalue problem.}

In the study of the rigidity of surfaces of revolution one encounters the following "double" eigenvalue problem [E. Rembs, Math. Zeit. vol. 35 (1932) pp. 529-535]: Find those numbers $a>0$ for which there exists a not identically vanishing function $y$ and an integer $m^{2}-1>0$ such that $y$ is a solution of the system $\left(^{*}\right)\left(p y^{\prime}\right)^{\prime}$ $=\lambda p^{2} q(a+r)^{-1} y=0, y(0)=y(b)=0$, with $\lambda=m^{2}-1$. Here $p, q$, and $r$ are analytic, nonnegative functions on $(0, b)$ and $p$ has a pole of the first order at the end points. Rembs demonstrated the existence of a countably infinite set of such "exceptional" values $a$, and Minagawa and Radó [to be published in Math. Zeit.] showed that these values are at most countable in number. For a fixed $a,\left(^{*}\right)$ is a regular singular Sturm-Liouville eigenvalue problem and the application of known theorems about the eigenvalues $\lambda_{n}(a)$ leads to the conclusion that the $a$ 's satisfying the original problem form a dense set on the positive real axis. (Received June 11, 1953.)

\section{Applied Mathematics}

659. R. C. F. Bartels ( $\mathrm{p}$ ) and A. C. Downing, Jr.: On surface waves generated by travelling disturbances with circular symmetry.

Kelvin's ship wave problem for the surface waves produced by a distribution of pressure moving with constant velocity on the surface of a body of water with infinite depth is formulated in terms of a singular integrodifferential equation. It is shown that the surface elevation of the wave, $\eta(x, y)$, satisfies the equation $\eta(x, y)$ $+p(x, y)+(1 / 2 \pi) \iint_{-\infty}^{+\infty}\left(1 /\left(\left(x-x^{\prime}\right)^{2}+\left(y-y^{\prime}\right)^{2}\right)^{1 / 2}\right) \partial^{2} / \partial x^{\prime 2} \eta\left(x^{\prime}, y^{\prime}\right) d x^{\prime} d y^{\prime}=0$ where $p(x, y)$ 
is proportional to the surface pressure. To this is added the condition: $\eta(x, y) \rightarrow 0$ as $x \rightarrow+\infty$ and $y \rightarrow \pm \infty$. The solution of the equation for $\eta$, taken by itself, is not unique; the solutions of the homogeneous equation correspond to the "free" surface waves. However, for the pressure distributions with circular symmetry that are treated, the boundary condition is sufficient to specify the solution completely. The expression for the predominant part of the surface wave produced behind the moving source of disturbance is obtained with the aid of the Abelian theorems for the complex inversion integral in the theory of Laplace transforms. (Received July 13, 1953.)

660t. Nathaniel Coburn: The intrinsic form of the characteristic relations in the steady supersonic motion of a fuid.

The purpose of this paper is to write the characteristic relations for the steady supersonic motion of a fluid in terms of curvatures associated with the characteristic manifolds, and the rates of change of the magnitude of the velocity and that of the sound speed with respect to displacements along the normal to the characteristic surfaces and along two independent directions in these manifolds. This is done for steady three-dimensional non-isentropic rotational flows. Canonical forms of these relations are obtained and application is made to plane rotational flows. Here it is shown that simple waves (straight line bicharacteristics) exist for the limiting case when the Mach number of the flow is one. Then, the stream lines are orthogonal to the straight line bicharacteristics, and the magnitude of the velocity and the sound speed are constant along a stream line. For general plane rotational flows, necessary and sufficient conditions for the existence of simple waves are obtained. (Received July 16, 1953.)

661. C. L. Dolph, J. E. McLaughlin, and Immanuel Marx (p): Symmetric linear transformations and complex quadratio form.

MacFarlane (Cambridge Philos. Soc. vol. 43 (1947)) devised a nonlinear variational approach to the complex eigenvalue problem occurring in the theory of a anomalous propagation. This paper treats the finite-dimensional aspects of the corresponding linear Rayleigh-Ritz procedure. In particular, a spectral theorem is derived for a class of symmetric linear transformations on an $n$-dimensional vector space over an algebraically closed field of characteristic $\neq 2$. If the vector space is nonsingular with respect to a symmetric bilinear form, a necessary and sufficient condition for such a theorem is that the transformations have minimum functions with simple roots. In physical applications the complex number field is used and the eigenvalues have the additional property that imaginary parts are restricted to one half-plane. Under these circumstances the imaginary parts are characterized as saddle points in suitably chosen subspaces in a manner similar to the minimum characterization of the eigenvalues in the case of real fields. (Received July 16, 1953.)

662. R. L. Evans: Errors in the asymptotic solution of linear ordinary differential equations.

A new method of estimating the error in an asymptotic approximation was suggested by a reading of T. Thorkelsson's Divergent power series (Reykjavik, 1934). Consider (1) $L(y)=\sum_{\nu=0}^{n} p_{\nu}(x) y^{(n-\nu)}=0$, where the $p_{\nu}$ 's are polynomials such that for one solution $y(x) \sim \sum_{\alpha=0}^{\infty} b(\alpha) x^{\rho-\alpha}$ in a sector $S$. Then the recurrence relation for the $b$ 's can be written as (2) $\sum_{\nu=0}^{m} q_{\nu}(\alpha, \rho) b(\alpha+m-\nu)=0$. Let $u(x)=\sum_{\alpha_{m-0}}^{N-1} b(\alpha) x^{\rho-\alpha}$ 
and (3) error $v(x)=y(x)-u(x)$. Then if $\rho=\sigma+N$ and $b(\alpha+N)=B(\alpha)$, (2) becomes $\sum_{\nu_{m=0}}^{m} q_{\nu}(\alpha, \sigma+N) B(\alpha+m-\nu)$ which corresponds to a differential equation (4) $M(v)$ $=0$. From (1), (3), and (4) one obtains (5) $M(v)-L(v)=L(u)$ which is a differential equation of order $(n-1)$ having $v(x)$ as a particular solution $\ni \lim _{x \rightarrow \infty}$ in $s\left|x^{N-1} v(x)\right|$ $=0$. When $n=2$, (5) is readily solved but when $n>2$ it is usually better to use the author's other error estimate (Proc. Amer. Math. Soc. vol.4 (1953)). The error depends on the last $m$ terms of $u(x)$ and is not necessarily least when $b(N-1) x^{\rho+1-N}$ is the smallest term. For (6) $L(y)=x^{3} y^{\prime \prime}+\left(A_{1} x+A_{2}\right) x^{2} y^{\prime}+\left(A_{3} x^{2}+A_{4} x+A_{6}\right) y=0\left(A_{1} \neq 0\right)$ a suitable contour integration of (5) gives $|v(x)|<\mid(N-\rho-1) \cdot\left((N-\rho)-A_{2}(N-1)\right) / N\left(A_{2}\right.$ $-2 \rho-N+1)|\cdot| T_{N-1}|+| A_{5} / N x\left(A_{2}+2 \rho-N-1\right)|\cdot| T_{N-1}|+| A_{6} / N x\left(A_{2}+2 \rho-N\right.$ $+1)|\cdot| T_{N-2} \mid$ where the $T^{\prime}$ 's are the last terms in $u(x)$. Equation (6) has the confluent hypergeometric equation and a modified Bessel equation as special cases involving $A_{5}=0$. This work was supported by the Office of Ordnance Research. (Received July 13, 1953.)

663. G. E. Forsythe: Arbitrarily close lower bounds for the fundamental frequency of a convex membrane.

Let $R$ be a convex plane region bounded by a piecewise sufficiently smooth curve $C$. Let $\lambda$ be the least number such that $\Delta u \equiv u_{x x}+u_{y y}=-\lambda u$ in $R$, with $u=u(x, y)=0$ on $C$. (Subscripts $x, y$ denote differentiation.) Let a square net $N_{h}$ of mesh-width $h$ be formed by parallels to the axes. Let $R_{h}$ denote the nodes of $N_{h}$ inside $R$; let $C_{h}$ be the intersection of $N_{h}$ with $C$. A self-adjoint finite-difference operator $\Delta_{h}$ approximating $\Delta$ is introduced; it is the symmetric part of the operator (65.1) in W. E. Milne, Numerical solution of differential equations, Wiley, 1953. Let $\lambda_{h}$ be the least number such that $\Delta_{h} v+\lambda_{h} v=0$ in $R_{h}$, where $v=v(x, y)$ is defined for $(x, y) \in R_{h} \cup C_{h}$ and vanishes on $C_{h}$. Theorem: As $h \rightarrow 0, \lambda_{h} / \lambda \leqq 1-h^{2}(A+B) / D+o\left(h^{2}\right)$. Here $A$ $=\iint_{R}\left(u_{x x}^{2}+u_{y y}^{2}\right) d x d y ; B=\int_{C} u_{n}^{2} \sin ^{2} 2 \tau d \tau ; D=12 \iint_{R}\left(u_{x}^{2}+u_{y}^{2}\right) d x d y ; u_{n}$ is the normal derivative of $u ; \tau$ is the angle between the tangent to $u$ and the $x$ axis; $u$ is the fundamental solution of $\left({ }^{*}\right)$. Thus ultimately $\lambda_{h} \uparrow \lambda$. This extends the result for certain polygonal regions $R$ announced and discussed in Bull. Amer. Math. Soc. Abstract 59-4-509. (Received July 13, 1953.)

664t. Isidor Heller: On the problem of shortest path between points. I.

The $n$ ! closed paths connecting $n$ given points are represented by the set $P_{n}$ of $n$ by $n$ permutation matrices. These, interpreted as points in $n^{2}$-space, are the extreme points of the convex set of doubly stochastic matrices (Birkhoff, von Neumann). In problem above only the subset $C_{n}$ consisting of the $(n-1)$ ! cycles of order $n$ is admitted. $C_{n}$ has dimension $(n-1)^{2}-n$ so that the 6 points of $C_{4}$ form a 5-dimensional simplex (H. Kuhn in a letter to author). A first objective was to determine the extreme hyperplanes of the convex of $C_{5}$, which is an 11-dimensional polytope in 25 -space with 24 vertices, the main difficulty opposing straightforward computation being the nearly 2.5 million possibilities of choosing 11 points out of 24 . Results: The convex of $C_{5}$ is characterized by a nonredundant system of 224 hyperplanes of the following 6 types. (1) $X_{i j} \geqq 0(i \neq j)$; (2) $X_{i i}=0$; (3) sum of any row=sum of any column $=1$ (one of the $2 n$ equations to be omitted in order to avoid redundancy); (4) $X_{i j}+X_{j i} \leqq 1$; (5) $X_{i j}+X_{j i}+X_{r s}-X_{s t}-X_{t r} \leqq 1$ for distinct $(i, j, r, s, t)$; (6) $2 X_{i j}$ $+2 X_{j i}-X_{i r}+X_{j r}-X_{s i}+X_{s j}-X_{r s} \leqq 2$ for distinct $(i, j, r, s)$. (Received June 24, 1953.)

665t. Isidor Heller: On the problem of shortest path between points. II. 
For general $n \geqq 5$ the conditions characterizing doubly stochastic matrices with zeros in the main diagonal and the condition that the elements of any principal minor of order $k \leqq n / 2$ should sum to at most $k-1$ represent a relatively small number of the extreme hyperplanes of the convex of $C_{n}$. More numerous classes are obtained through a relation transforming certain types of hyperplanes of the cases $m<n$ into such of the case $m=n$; so that for instance the 120 hyperplanes of type (6) of the convex of $C_{5}$ (see paper above) determine 1800 extreme hyperplanes of the convex of $C_{6}$. The problem of shortest closed path connecting $n$ points and the problem of shortest nonclosed path (with distinct beginning and end point) connecting $n-1$ points are equivalent in the sense that there exists a nonsingular linear transformation mapping the convex of all possible paths of the first kind onto the convex of all possible paths of the second kind. (Received June 22, 1953.)

666t. M. Z. Krzywoblocki: Generalization of Coburn's "independent scalars" in homogeneous turbulence to compressible media.

Coburn furnished a method for the construction of independent scalars in various types of homogeneous turbulence in incompressible fluids. By definition, these scalars must satisfy, in addition to other conditions, continuity relations identically, that is, these tensors must have zero divergence. Briefly, they are solenoidal tensors. But, as was proved by the author in many of his papers, the tensors in the theory of turbulence in compressible media are not any longer solenoidal, and their divergence must be equal "identically" to some given function. The present note discusses the changes which must be introduced into Coburn's paper in order to obtain a generalization of his method of the construction of independent scalars for homogeneous turbulence in compressible media. (Received July 6, 1953.)

\section{F. M. Reza: Some new applications of the theory of a polynomial in a complex variable to electrical network theory.}

The notions of derivative of a polynomial and location of its critical points have seldom been used in electrical engineering problems. The object of this paper is to provide a new connection between the mathematics of polynomials and the electrical network theory. The following two key theorems serve this purpose by connecting the class of positive real functions (Brune functions) used in electrical network theory, to the theory of the location of critical points of polynomials in a complex variable. Theorem 1: If $P(s) / Q(s)$ represents the driving-point impedance of a one terminalpair lossless network (or a two-element type dissipative network), then $\left(d^{n} P(s) / d s^{n}\right) /\left(d^{n} Q(s) / d s^{n}\right)$ will also represent a one terminal-pair lossless network (or a two-element type dissipative network). Theorem 2: The logarithmic derivative of any Hurwitz polynomial is a positive real function. Network configurations corresponding to the above theorems are given. To demonstrate some introductory uses for the above theorems, a lemma of Laguerre is first proved by electrical analogy; then some formulas are discussed for generating $R C$ polynomials from a given $R C$ polynomial. (Received June 29, 1953.)

\section{8t. H. E. Salzer: New formulas for facilitating osculatory inter- polation.}

Hermite's osculatory interpolation formula employing the function $f(x)$ and its derivative $f^{\prime}(x)$ at $n$ equally spaced arguments $x_{i} \equiv x_{0}+i h, i$ ranging from $-[(n-1) / 2]$ to $[n / 2]$, is expressible in the form (1) $f\left(x_{0}+p h\right)=\sum_{i}\left(\left\{L_{i}^{(n)}(p)\right\}^{2}\left\{1-2 L_{i}^{(n) \prime}(i)(p-i)\right\} f_{i}\right.$ 
$\left.+\left\{L_{i}^{(n)}(p)\right\}^{2}(p-i) h f_{i}^{\prime}\right)+R_{2 n}(p), \quad$ where $\quad x=x_{0}+p h, \quad f_{i} \equiv f\left(x_{i}\right), \quad f_{i}^{\prime} \equiv f^{\prime}\left(x_{i}\right), \quad L_{i}^{(n)}(p)$ $=\prod_{j=-[(n-1) / 2]}^{\prime(n / 2]}(p-j) / \prod_{j=-[(n-1) / 2]}^{\prime(n / 2]}(i-i) \quad\left(j=i\right.$ absent from $\left.\Pi^{\prime}\right)$ and $R_{2 n}(p)$ $=f^{(2 n)}(\xi) h^{2 n}\left\{\prod_{j=-[(n-1) / 2]}^{[n / 2]}(p-j)\right\}^{2} /(2 n)$ ! for $x_{-[(n-1) / 2]} \leqq \xi \leqq x_{[n / 2]}$. Formula (1) is very much more accurate than the corresponding $n$-point Lagrangian formula and considerably more accurate than even the $2 n$-point Lagrangian formula for $x_{i}$ at intervals of $h$. Also (1) is specially suited for interpolation in many functions (e.g., Bessel, probability) that are tabulated with their derivative. To avoid the tremendous amount of labor in calculating the coefficients of $f_{i}$ and $f_{i}^{\prime}$ in the form given in (1), it is shown that we may express (1) as (2) $f\left(x_{0}+p h\right) \sim \sum_{i}\left(\alpha_{i} f_{i}+\beta_{i} h f_{i}^{\prime}\right) / \sum \alpha_{i}+R_{2 n}(p)$, where $\alpha_{i} \equiv a_{i} /(p-i)^{2}+b_{i} /(p-i), \beta_{i} \equiv a_{i} /(p-i)$, and where $a_{i}=k(n) /\left\{\prod_{j=-[(n-1) / 2]}^{\prime(n / 2\}}(i-j)\right\}^{2}$, $b_{i}=-2 L_{i}^{(n) \prime}(i) a_{i}$, the $k(n)$ being any suitably chosen constant of proportionality depending only upon $n$ (here chosen to make $a_{i}$ and $b_{i}$ integers). The exact values of $a_{i}$ and $b_{i}$ are given for $n=2(1) 11, i=-[(n-1) / 2]$ to $[n / 2]$, so that Hermite's formula can be applied exactly for any polynomial up to the 21 st degree. A schedule gives approximate upper bounds for the coefficients of $f^{(2 n)}(\xi) h^{2 n} \sim \Delta^{2 n} f(x)$ in $R_{2 n}(p)$. (Received May 15, 1953.)

669. H. E. Salzer: Orthogonal polynomials arising in the numerical evaluation of inverse Laplace transforms.

In finding $f(t)$, the inverse Laplace transform of $F(p)$, where (1) $f(t)$ $=(1 / 2 \pi j) \int_{c-i \infty \infty}^{c+j \infty} e^{p t} F(p) d p$, the function $F(p)$ may be either known only numerically or too complicated for evaluating $f(t)$ by Cauchy's theorem. When $F(p)$ behaves like a polynomial without a constant term, in the variable $1 / p$, along $(c-j \infty, c+j \infty)$, one may find $f(t)$ numerically using new quadrature formulas (analogous to those employing the zeros of the Laguerre polynomials in the direct Laplace transform). Suitable choice of $p_{i}$ yields an $n$-point quadrature formula that is exact when $\rho_{2 n}$ is any arbitrary polynomial of the $(2 n)$ th degree in $x \equiv 1 / p$ without a constant term, namely: (2) $(1 / 2 \pi j) \int_{c-j \infty \infty}^{c+j \infty} e^{p} \rho_{2 n}(1 / p) d p=\sum_{i=1}^{n} A_{i}^{(n)} \rho_{2 n}\left(1 / p_{i}\right)$. In (2), $x_{i} \equiv 1 / p_{i}$ are the zeros of the orthogonal polynomials $p_{n}(x) \equiv \prod_{i=1}^{n}\left(x-x_{i}\right)$ where $(3) \quad(1 / 2 \pi j)$ $\cdot \int_{c-j \infty}^{c+j \infty} e^{p}(1 / p) p_{n}(1 / p)(1 / p)^{i} d p=0, i=0,1, \cdots, n-1$, and $A_{i}^{(n)}$ correspond to the Christoffel numbers. The normalization $P_{n}(1 / p) \equiv(4 n-2)(4 n-6) \cdots 6 p_{n}(1 / p), n \geqq 2$, produces all integral coefficients. $P_{n}(1 / p)$ is proved to be $(-1)^{n} e^{-p} p^{n} d^{n}\left(e^{p} / p^{n}\right) / d p^{n}$. The normalization factor is proved, in three different ways, to be given by (4) $(1 / 2 \pi j) \int_{c-j \infty}^{c+j \infty} e^{p}(1 / p)\left[P_{n}(1 / p)\right]^{2} d p=(-1)^{n} / 2$. Proofs are given for the recurrence formula (5) $(2 n-3) P_{n}(x)=[(4 n-2)(2 n-3) x+2] P_{n-1}(x)+(2 n-1) P_{n-2}(x)$, for $n \geqq 3$, and the differential equation $(6) x^{2} P_{n}^{\prime \prime}(x)+(x-1) P_{n}^{\prime}(x)-n^{2} P_{n}(x)=0$. The quantities $p_{i}^{(n)}, 1 / p_{i}^{(n)}$, and $A_{i}^{(n)}$ were computed, mostly to $6 S-8 S$, for $i=1(1) n, n=1(1) 8$. (Received June 4, 1953.)

\section{0t. H. H. Snyder: A class of Galerkin vectors. Preliminary report.}

Let $\bar{\rho}=i \xi+j \eta+k \zeta$ be the displacement vector of a point in the interior of a perfectly elastic body, and let $\bar{K}$ be the vector body force. The equation to determine $\bar{\rho}$ is $\left(\nabla^{2}+a \nabla \mathrm{div}\right) \bar{\rho}+b \bar{K}=0$ (cf. Westergaard, Theory of elasticity and plasticity), where $a$ and $b$ are constants of the material. Galerkin and Papcovitch have shown that $\bar{\rho}=b\left[(1-\mu) \nabla^{2}-(1 / 2) \nabla \operatorname{div}\right] \bar{F}$ provided that $\nabla^{4} \bar{F}+\bar{K} /(1-\mu)=0$. If $K=0$, then $\vec{F}=i f_{1}+j f_{2}+k f_{3}$ is a biharmonic vector function, and hence the $f_{k}(x, y, z)$ are biharmonic scalar functions. An algebra $A$ is defined such that the components of monogenic functions over $A$ are scalar biharmonic functions. Let $V$ be the set of all vectors in three dimensions. Functions $\bar{G}: A \rightarrow V$ are defined in such a way that the $\bar{G}$ are all biharmonic vector functions. A theory of functions for this class of Galerkin 
vectors is developed, and the three-dimensional states of stress defined by them are analyzed. (Received July 17, 1953.)

671. W. S. Snyder: A method of obtaining bounds for the solution of certain functional equations.

If a Volterra integral equation has a non-negative kernel and if iteration decreases a function $g$ at each point, then $g$ and its iterate are upper bounds for the solution of the integral equation. An estimate for the difference of $g$ from the solution is obtained. Various methods of obtaining such upper bounds are considered. Similar results for lower bounds are immediate and the method is extended to certain types of differential-difference equations. (Received July 20, 1953.)

\section{Domina E. Spencer: Field theory and the new electrodynamics.}

A single Galilean-relativistic equation for the force between moving charges has been derived. This equation provides the basis of a new electrodynamics which treats adequately the principal results of Maxwell's theory while obviating the necessity of introducing a theory of special relativity. The implications of the equation for field theory and the introduction of the scalar and vector potential are discussed in this paper. (Received July 17, 1953.)

673t. G. L. Spencer, II : Existence of Taylor-Maccoll flow and related inequalities.

The Taylor-Maccoll equations for the flow about a right circular cone immersed without yaw in a uniform flow such that an attached shock wave is formed are considered. From the local existence of solutions of the equations which meet the conditions on the cone the existence in the large of solutions which also meet the shock conditions is obtained provided that the angle $\theta$, the normalized velocity $u$ on the body, and the gas constant $\gamma$ satisfy the inequalities $\left[\gamma-1+2 \sin ^{2} \theta\right] \geqq \gamma-1$ and $(\gamma-1) u+(\gamma+1)(1-u)^{1 / 2}$ arc $\cos \theta \geqq(\gamma+1) \theta\left(1-u^{2}\right)^{1 / 2}$. This work was sponsored by Army Office of Ordnance Research Contract No. DA-36-034-ORD-966. (Received July 10, 1953.)

674. G. L. Spencer, II : The compressible flow about a pointed body of revolution of curved profile.

The equations and boundary conditions for the rotational flow about a pointed body of revolution of curved profile which is immersed without yaw in a uniform flow such that an attached shock wave is formed are considered. If the body profile given in polar coordinates by $\theta=B(r)$ is cone-like in the sense that $B^{(2 n-1)}(0)=0, n$ $=1,2, \cdots$, and certain inequalities are satisfied, then series for the velocity components, the entropy functions, and the shock profile are obtained. These series constitute a formal solution since it can be shown that if they converge they represent a solution. This work was sponsored by Army Office of Ordnance Research Contract No. DA-36-034-ORD-966. (Received July 10, 1953.)

\section{GEOMETRY}

\section{5t. Louis Auslander: Curvature in Finsler geometry.}

If a manifold $M$ has a Finsler metric $F$, then the sphere bundle (space of line elements) $S$ over $M$ has a natural induced Riemannian metric $R$. The metric $R$ has the 
following properties: (a) the distance between any two points in $S$ is greater then or equal to the distance between the projections of these points in $M$; (b) by associating with a geodesic its tangent directions, one gets a curve in $S$ which is a geodesic in $S$ with respect to $R$ and which has the same length in $S$ as in $M$. By the use of a satisfactory definition of sectional and mean curvature one can prove the following: (1) A generalization of Myers' theorem holds, i.e., a complete $n$-dimensional Finsler manifold whose mean curvature is $\geqq e^{2}>0$ is compact and has diameter $\leqq \pi / e$. From this it follows that the fundamental group of $M$ is finite. (2) A generalization of Synge's Theorem holds, i.e., an even-dimensional Finsler metric of positive sectional curvature is simply connected. (3) Let $M$ be a complete simply connected manifold with sectional curvature $\leqq 0$; then $M$ is homeomorphic to Euclidean space. This definition of negative curvature agrees with the one given by Busemann in Acta Math. vol. 80, pp. 259-310. (Received April 23, 1953.)

676t. L. M. Blumenthal: On an extension of a theorem of Jordan and von Neumann.

The theorem referred to proves that euclidean spaces of finite or infinite dimensions are characterized among all complete normed linear spaces $L$ by the validity of the norm relation $\|f+g\|^{2}+\|f-g\|^{2}=2\left(\|f\|^{2}+\|g\|^{2}\right), f, g \in L$. The relation is metrically equivalent in $L$ to the following (feeble euclidean) four-point property: if $p, q, r \in L$ with $q$ a middle-element of $p, r$, then for each $s \in L, p, q, r, s$ are congruently contained in the euclidean plane $E_{2}$. It is shown in this paper that in complete, convex, externally convex, metric spaces $\{M\}$ this property is equivalent to the weak euclidean four-point property introduced by the writer in 1934, and hence it characterizes finite or infinite-dimensional euclidean spaces among the much larger class of spaces $\{M\}$ (see L. M. Blumenthal, Theory and applications of distance geometry, Oxford, Clarendon, 1953, pp. 123-129). (Received May 1, 1953.)

677. W. M. Boothby: Some fundamental formulas for Hermitian manifolds.

The equations of structure of a complex-analytic manifold $M$ with Hermitian metric are: $d \omega^{i}=\omega^{k} \omega_{k}^{i}+\Omega^{i} ; d \omega_{j}^{i}=\omega_{k}^{i} \omega_{j}^{k}+\Omega_{j}^{i}$ where the forms $\omega^{i}, \omega_{j}^{i}$ are uniquely defined in the bundle $B$ of orthonormal frames over $M$ (S. S. Chern, Ann. of Math. vol. 47 (1946) pp. 85-121). Every tensor or tensorial form on $M$ has well defined components relative to each frame, components which become functions on $B$ if we allow the frame to vary over all orthonormal ones, giving components on $B$ of each tensor of $M$. Using these components and the equations of structure, the tensor calculus of $M$ may be transferred to $B$, a general technique of Chern which in this instance is developed in detail for the case of Hermitian manifolds. Formulas, including expressions for $d \phi$ and $\delta \phi$ which in the Kaehler case are similar to those given by $\mathrm{H}$. Guggenheimer (Comment. Math. Helv. vol. 25 (1951) pp. 257-297), are derived and applications are given. (Received July 13, 1953.)

\section{8t. John DeCicco: Dynamical systems of $\infty^{3}$ conic sections.}

If a positional field of force possesses at least $3 \infty^{2}$ proper conics as dynamical trajectories, then all are conics. If the lines of force are all straight, and if the field possesses at least $2 \infty^{2}$ proper conics as trajectories, then all are conics. In the projective plane, a dynamical system of $\infty^{3}$ conics consists of either: (I) all the conics for which a fixed point and line are pole and polar, or: (II) all the conics tangent to two distinct lines, real or imaginary. In the Euclidean plane, a dynamical family of $\infty^{3}$ 
ellipses consists of four types. The period is obtained for every elliptic trajectory in each of the four associated parallel or central fields of force. In each, there are $\infty^{2}$ elliptic trajectories for which the square of the period is directly proportional to the cube of the mean distance. The Newtonian field of force is characterized by the following weakened form of Kepler's laws on planetary motion: (1) the field of force contains $\infty^{3}$ ellipses as trajectories, and (2) for each of the $\infty^{3}$ ellipses, the square of the period varies directly as the cube of the mean distance. (Received April 27, 1953.)

679t. David Gale: Separation theorems for families of convex sets.

The classical theorem on the separation of disjoint convex sets by a hyperplane may be rephrased as follows: If $C_{1}$ and $C_{2}$ are disjoint open (compact) convex sets in $n$-space, then there exist disjoint open (closed) halfspaces $H_{1}$ and $H_{2}$ such that $C_{1} \subset H_{1}$ and $C_{2} \subset H_{2}$. In this form the result can be generalized as follows. Theorem: Let $\left\{C_{\alpha}\right\}$ be an indexed family of open (compact) convex sets in $n$-space, $\alpha \in A$, such that $\bigcap_{\alpha \in A} C_{\alpha}=\varnothing$. Then there exists a family $\left\{H_{\alpha}\right\}$ of open (closed) halfspaces such that $C_{\alpha} \subset H_{\alpha}$ and $\bigcap_{\alpha \in A} H_{\alpha}=\varnothing$. The proof when the $C_{\alpha}$ are compact follows easily from the classical theorem. When the $C_{\alpha}$ are open and the family $\left\{C_{\alpha}\right\}$ has the finite intersection property, the proof is considerably more difficult. (Received July $8,1953$.

680. W. R. Hutcherson: Concerning some cyclic involutions with periods $p \leqq 157$.

Lucien Godeaux [Sur quelques surfaces algêbriques représentant des involutions cycliques, Bulletin de l'Academie royale de Belgique (1951) pp. 1106-1118] and other authors have studied cyclic involutions with certain prime periods $p \leqq 73$. The writer [W. R. Hutcherson, Voisage du cinquiême ordere d'une involution de période 13, Bulletin Royale des Sciences de Liège, No. 11 (1952) pp. 483-487] has studied a fourth degree surface where the involutions contained thereon were of period $p \leqq 13$. However, this present paper exhibits a fifth degree surface invariant under a cyclic homography $x_{1}^{\prime}: x_{2}^{\prime}: x_{3}^{\prime}: x_{4}^{\prime}=x_{1}: \epsilon x_{2}: \epsilon^{10} x_{3}: \epsilon^{8} x_{4}$, where $\epsilon^{13}=1$. When $p=157$, a surface of order fourteen was found. It was invariant under the homography $x_{1}^{\prime}: x_{2}^{\prime}: x_{3}^{\prime}: x_{4}^{\prime}$ $=x_{1}: \epsilon x_{2}: \epsilon^{145} x_{3}: \epsilon^{101} x_{4}$ where $\epsilon$ is the one hundred and fifty-seventh primitive root of unity. Only three points of coincidence appeared on this surface. Certain invariant space curves of order (157)(14) on this surface were found to pass through these three invariant points as twelve tuple points. (Received July 13, 1953.)

681. N. D. Lane (p) and Peter Scherk: Characteristic and order of differentiable points in the conformal plane.

In an earlier paper, the authors defined differentiable points on an $\operatorname{arc} A$ in the conformal plane. They described the various types of interior differentiable points $s$ by means of a one-row matrix $\left(a_{0}, a_{1}, a_{2}\right)$, the characteristic of $s$. In the present paper they prove: (1) The cyclic order of $s$, i.e., the maximum number of points which a small neighborhood of $s$ can have in common with any circle, is not less than $a_{0}+a_{1}+a_{2}$. (2) If $s$ decomposes $A$ into two arcs of cyclic order three, then the order of $s$ is equal to $a_{0}+a_{1}+a_{2}$. In order to prove (2) they establish a strong kind of differentiability at an end point of an arc of cyclic order three. (Received July 13,1953.)

\section{LOGIC AND FoundaTIONS}

682. P. C. Gilmore: On axioms related to the principle of the excluded third. 
It has been shown by P. C. Gilmore (Neder. Akad. Wetensch. vol. 56, No. 2, Ser. A) that the effect of Griss' criticism of the intuitionistic logic can be expressed by adding $(E x) p(\underline{x}) \bigvee(A \underline{x})\rceil p(\underline{x})$, which we denote by $A$, as an axiom of the logic for every atomic proposition or predicate $p$. It is worthwhile discussing the relations between $A$ and other expressions related to $B$, the principle of the excluded third, and the principle of the excluded third itself $((A \underline{x}) \cdot P(\underline{x}) \vee \neg P(\underline{x})$ for any wff $P)$. Consider also the expressions $C, D$ and $E$ which are defined to be respectively, $(A x)$ - $p(\underline{x}) \bigvee\rceil p(\underline{x})$ and $(A \underline{x}) p(\underline{x}) \bigvee(A \underline{x})\rceil p(\underline{x})$, for any atomic predicate or proposition $p$, and $(A x) \cdot Q(x) \bigvee \backslash Q(x)$, for any wff $Q$ constructed from the atomic predicates and propositions without the aid of quantifiers. It is not difficult to prove that $D$ implies $B$, that $C$ implies $E$, and that $B$ implies $A, C$, or $E$. One can also prove that $A$ and $C$ are independent. (Received July 10, 1953.)

683t. Theodore Hailperin: Quantification theory for empty individual domains.

A set of axioms for quantification formulas valid in all individual domains, including the empty domain, is presented. The system resulting is different from, and more "natural" than, that of Mostowski (Journal of Symbolic Logic vol. 16 (1951) pp. 107-111) in that far fewer of the usual quantification formulas become invalid. The difference between this system and Mostowski's arises from unlike treatments of vacuous quantification (quantifaction over formulas not containing the variable which appears in the quantifier). The axioms are so phrased that by the addition of one further axiom the usual quantification theory for formulas valid in all nonempty domains is obtained. A simple proof of completeness is given. (Received April 20, 1953.)

\section{4t. S. C. Kleene and E. L. Post: The upper semi-lattice of degrees of recursive unsolvability.}

The degrees of recursive unsolvability (Post, Bull. Amer. Math. Soc. Abstract 54-7-269) form an upper semi-lattice under a l.u.b. operation $a \bigcup_{b}$, but not a lattice. To any degree $\boldsymbol{a}$ (including the degree $\boldsymbol{O}$ of a recursive set), let $\boldsymbol{a}^{\prime}$ be the degree of the complete $A$-canonical set (Post, loc. cit.) or equivalently of the predicate $(E x) T_{1}^{A}(a, a, x)$ (Kleene, Introduction to metamathematics, Chapter $\mathrm{XI}$ ) where $A$ is of degree $\boldsymbol{a}$; and let $\boldsymbol{a}^{(\omega)}$ be the degree e.g. of the predicate $M^{A}(a, k)$ (Kleene, loc. cit.). There exist between $\boldsymbol{a}$ and $\boldsymbol{a}^{\prime}$ (between all $\boldsymbol{a}, \boldsymbol{a}^{\prime}, \boldsymbol{a}^{\prime \prime}, \cdots$ and $\boldsymbol{a}^{(\omega)}$ ) infinitely many degrees pairwise incomparable, and also e.g. infinitely many degrees ordered among themselves as the rational numbers. (Received July 17, 1953.)

\section{H. G. Rice: Recursive real numbers.}

The four definitions of Specker (J. Symbolic Logic vol. 14 (1949) pp. 145-158) are stated, using general recursive functions, and the fact of their equivalence mentioned (Robinson, J. Symbolic Logic vol. 16 (1951) p. 282). The following theorems are stated and proofs outlined. 1. There is no effective general method for deciding whether or not two recursive real numbers are equal. However, two unequal numbers can be effectively compared for size. 2 . The recursive real numbers form a field. 3 . The field of recursive real numbers is closed under sequences whose convergence is given by a recursive function. 4 . The field of recursive complex numbers is algebraically closed. The last proof is adapted from Rosenbloom (Amer. Math. Monthly vol. 52 (1945) pp. 562-570). (Received July 15, 1953.)

686t. Hao Wang: Constructive foundations of mathematics. I. 
A theory $\boldsymbol{\Sigma}$ is presented such that for every ordinal number $\alpha$ of Cantor's second number class, there are sets of level $\alpha$. On the 0th level are the finite sets built up from the empty set. On the $(\beta+1)$ th level are all the sets of level $\beta$ plus all sets of them which can be defined by properties referring at most only to the totality of all sets of level $\beta$. If $\alpha$ is a limiting number, then sets of level $\alpha$ include all and only the sets of levels lower than $\alpha$. The theory $\Sigma$ is determined by a schema of axiomatization such that for each given $\alpha$, a formal system $\Sigma_{\alpha}$ dealing with sets of level $\alpha$ is given. To put it in another way, $\boldsymbol{\Sigma}$ is the union of all such systems. A modelling proof of consistency and a proof-theoretic one are given; the latter is apparently "finitist." The theory includes strong axioms of limitation. (Received July 16, 1953.)

\section{7t. Hao Wang: Constructive foundations of mathematics. II.}

All sets in $\Sigma$ are enumerable in $\Sigma$ so that Skolem's paradox does not occur. Consistency of each system $\boldsymbol{\Sigma}_{\alpha}$ is provable in $\boldsymbol{\Sigma}_{\alpha+2}$. Therefore, Gödel's theorems do not apply to the whole theory $\boldsymbol{\Sigma}$. Axioms of choice and forms of the continuum hypothesis are provable in $\boldsymbol{\Sigma}$. Ordinary theories of natural and real numbers can be obtained in $\Sigma$, including such standard theorems as the least upper bound, the limit point, the finite covering. It is contended that, although this theory $\boldsymbol{\Sigma}$ would not satisfy Brouwer who seems to permit nothing but the effectively decidable, it should satisfy Hilbert who only asks to be finitist in the metamathematics which justifies the system actually used by a proof of consistency. Moreover, the theory $\Sigma$ is natural in that it includes all the standard principles such as the tertium non datur and the axiom of extensionality. This is taken as a decisive advantage over most of the alternative reconstructions of mathematics. (Received July 16, 1953.)

\section{Statistics ANd Probability}

688t. K. L. Chung: Contributions to the theory of Markov chains. II.

Let $\left\{X_{n}\right\}, n \geqq 0$, be a Markov chain with stationary transition probabilities and with the non-negative integers as states forming one recurrent class. Let $f$ be a realvalued function on the non-negative integers. The main purpose of this paper is to study the sequence $\left\{f\left(X_{n}\right)\right\}, n \geqq 0$. We obtain, in turn, an ergodic theorem of the Hopf type, weak and strong laws of large numbers, the central limit theorem (due to Doblin), the law of the iterated logarithms, and the limit theorems for the maxima of $\sum f\left(X_{n}\right)$. These results are proved very simply by exploiting an idea of Doblin's and. they are applicable to a finite (recurrent) chain without any condition whatever. The conditions of validity in the denumerable case deserve attention. Among other things it is shown by an example that a certain set of conditions, attributed to Kolmogorov, is in reality not sufficient for the validity of the central limit theorem. Furthermore, conditions of validity for the strong limit theorems and the limit theorems for the maxima are obtained by a natural strengthening of the corresponding conditions for the weak limit theorems. (Received June 24, 1953.)

\section{9t. T. E. Harris: On chains of infinite order.}

Let $z_{n}, n=0, \pm 1, \cdots$, be a stationary stochastic process with $D$ possible values $0,1, \cdots, D-1$. Completing work of Doeblin and Fortet, Onicescu and Mihoc, and others on "chaînes à liaisons complètes" it is shown that such a process exists satisfying $P\left(z_{n+1}=i \mid z_{n}=u_{0}, z_{n-1}=u_{1}, \cdots\right)=Q_{i}(u)$ with probability 1 , where the $Q_{i}$ are given functions of $u=\left(u_{0}, u_{1}, \cdots\right)$ satisfying certain conditions. The quantity $x_{n}=\sum_{k=0}^{\infty} z_{n-k} / D^{k+1}$ uniquely determines the past of $z_{n}$ with probability 1 . The dis- 
tribution $G(x)$ of the past must be one of the three types (1) a step function with one step; (2) $G(x)=x, 0 \leqq x \leqq 1$, this being the case if and only if the $z_{n}$ are independent for different values of $n$, each being equidistributed; (3) purely singular. Special results on the prediction problem are obtained for the following type of process: let $y_{n}$ be a stationary Markov chain with a finite number of states divided into $D$ groups of states $G_{i}$; let $z_{n}=i$ if $y_{n} \in G_{i}$. The methods depend upon the fact that the past, $x_{n}$, is a special type of Markov process recently used as a model for learning processes by a number of authors. (Received July 16, 1953.)

690t. M. S. Klamkin: On the uniqueness of the distribution function for the Buffon needle problem.

In the well known Buffon needle problem the probability that a needle of length $L$, when dropped on a board ruled with equidistant parallel lines of spacing $D(D \geqq L)$, will intersect one of the lines is given by $P=2 L / \pi D$. This result has been derived by Barbier in a very elegant method not depending upon a distribution function. It is then shown that the distribution function in $x$ and $\phi$ (where $x$ is the distance from the needle's midpoint to the nearest line and $\phi$ is the acute angle between this distance and one of the ruled lines) is not unique. However, if $x$ and $\phi$ are assumed to be independent variables, then it is shown that the distribution in $x$ is uniform and thus unique, and that the distribution in $\phi$ is not unique. If the problem is made slightly more involved by taking $D \leqq L$, then it can be shown that there is only one distribution function which leads to the probability one would obtain by assuming a uniform distribution in $x$ and $\phi$. (Received July 17, 1953.)

\section{1t. Gerhard Tintner: Stochastic linear programming.}

Linear programming deals with the maximization of a linear form under linear inequalities. These inequalities can be transformed into equalities by the use of slack variables. We maximize $\phi=a^{\prime} x$ ( $a$ and $x$ column vectors of $n$ components) under the conditions: $B x=c$ ( $B$ a matrix, $c$ a column vector of $m$ components) and $x \geqq 0$. Select $m$ values of $a, x, c$ and $m$ columns of $B$. Denote the selection $B y^{(k)}$. Then $\phi^{(k)}=a^{(k)} x^{(k)}$ requiring $x^{(k)}=B^{(k)-1} c^{(k)} \geqq 0$. The $x$ is chosen for which $\phi^{*}=\max \phi^{(k)}$. Let the probability distribution of the elements of $a, B, c$ be $P(a, B, c ; w)$ where $w$ is a set of controlled parameters. From $P$ we derive the probability distribution of the maximand: $Q\left(\phi^{*} ; w\right)$. We maximize the functional $g[Q(\phi ; w)]$ with respect to $w$. A special case is $g=E \phi^{*}$, the mathematical expectation of the maximand. (Received June 18, 1953.)

\section{TOPOLOGY}

692. R. D. Anderson: Some upper semi-continuous collections of continuous curves filling up $R^{3}$.

The author establishes that for any positive integer $p$, there exists an upper semicontinuous collection $G$ of mutually exclusive locally connected continua filling up $R^{3}$ (the 3-cell) such that $G$ with respect to its elements as points is homeomorphic to $R^{p}$. This result is a strengthening of (and is to be published with) the author's previously announced result (Bull. Amer. Math. Soc. Abstract 59-3-319) that for any positive integer $p$, there exists a monotone mapping of $R^{3}$ onto $R^{p}$. (Received July 17 , 1953.)

\section{R. G. Bartle: Weak compactness in function spaces.}

A directed system $\left(f_{a}\right)$ of functions converges quasi-uniformly on a set $X$ to $f_{0}$ iff 
it converges on $X$ and, given $\epsilon>0, a_{0}$ there exist $a_{1}, \cdots, a_{n} \geqq a_{0}$ such that $X$ $=\bigcup_{i=1}^{n}\left\{x ;\left|f_{a_{i}}(x)-f_{0}(x)\right|<\epsilon\right\}$. If $X$ is compact Hausdorff and $f_{a} \in C(X)$, then $f_{0} \in C(X)$ iff the convergence is quasi-uniform on $X$. A set $\mathfrak{F} \subset C(X)$ is quasi-equicontinuous on $X$ iff every directed system $\left(x_{b}\right)$ in $X$ converging to $x_{0}$ converges quasi-uniformly on $\mathfrak{F}$. Theorem: A set $\mathfrak{F}$ is conditionally compact in the pointwise topology of $C(X)$ iff $\mathfrak{F}$ is pointwise bounded and quasi-equicontinuous on $X$. A subset of the Banach space $C(X)$ is conditionally compact in the weak topology iff it is bounded and quasi-equicontinuous. Similar conditions apply to the Banach space of bounded functions on an abstract set. Applications are made to weakly compact operators. These notions and results stem from work of Arzelà, Tychonoff, Sirvent, and Šmulian. (Received May 28, 1953.)

\section{W. R. Baum: On the two-dimensional universal minimal cycles.}

Let $M^{n}$ be an $n$-dimensional closed oriented manifold. A 2-dimensional cycle $Z^{2}$ in $M^{n}$ is said to be minimal if the intersection cycle $U^{n-1} \cdot Z^{2}$ is null-homologous in $M^{n}$ for all the $(n-1)$-dimensional cycles $U^{n-1}$ in $M^{n}$. (All cycles taken with respect to the integers as coefficients.) H. Hopf has shown (Comment. Math. Helv. vol. 14 (1941) pp. 257-309) that the group $\mathscr{X}_{2}$ of the homology classes of the minimal 2-cycles modulo the subgroup $\mathcal{L}^{2}$ of the homology classes of the cycles which are 2 -sphere images is determined by the fundamental group $G$ of $M^{n}$. If $U^{n-1} \cdot Z^{2} \sim 0$ holds not only for all the integral cycles $U^{n-1}$ but also for every $U^{n-1}$ which is a cycle $\bmod m(m=2,3,4, \cdots)$, then the (integral) cycle $Z^{2}$ is called a 2-dimensional universal minimal cycle in $M^{n}$. Let $G \cong \mathcal{F} / R$ be a representation of the fundamental group $G$ by a free group $\mathcal{F}$, and $\mathcal{C}^{2}$ the second commutator-subgroup of $\mathcal{F}$. Then one can prove the theorem: $Z^{2}$ is then and only then universal minimal if $Z^{2}$ has a homotopy boundary which represents an element of the group $C^{2}$. From this theorem follows: The homology group $\tilde{\mathscr{X}}^{2}$ of the universal minimal cycles $Z^{2}$ modulo the group $\mathcal{L}^{2}$ depends only on the fundamental group $G$. The dependence can be described explicitly by an isomorphism between the group $\tilde{\mathscr{T}}^{2} / \mathcal{L}^{2}$ and a certain group which depends only on $G$. (Received July 13, 1953.)

695. T. A. Botts, E. E. Floyd (p), and E. J. McShane: Lattices with pathological order topologies.

If $P$ is a partially ordered set, then, using the order topology, $P$ can also be considered as a topological space. It is easy to show that $P$ is then a $T_{1}$-space. Various examples are exhibited in which further separation properties do not hold. One example is of a complete, infinitely distributive lattice in which the open sets have the finite intersection property. Hence there exists a net in $P$ which converges, in the order topology, to every point of $P$. It is also shown that there exists a lattice group which is not Hausdorff in its order topology. It then follows that the group operations are not continuous. (Received July 20,1953.)

\section{6t. E. R. Fadell: Fixed point free mappings. Preliminary report.}

Let $\phi$ denote the fixed point property and $\bar{\phi}$ its negation. A topological space $X$ is said to be strongly $\bar{\phi}$ if there exists a fixed point free mapping from $X$ onto itself. Clearly strongly $\bar{\phi}$ implies $\bar{\phi}$. Examples show that strongly $\bar{\phi}$ and $\bar{\phi}$ are not equivalent on continua or on connected, locally connected spaces. It is well known that every multicoherent Peano continuum is $\bar{\phi}$. By a slight variation in the Kuratowski proof it is shown that such a space is strongly $\bar{\phi}$. Hence strongly $\bar{\phi}$ and $\bar{\phi}$ are equivalent on 
this class of spaces. The question as to the equivalence of strongly $\bar{\phi}$ and $\bar{\phi}$ for the general Peano continuum remains open. (Received July 16, 1953.)

\section{7t. Jean B. Feidner: Topological properties of a linear neighbor- hood space.}

In this investigation of a linear neighborhood space, any neighborhood $U_{x}$ of $x$ is defined thus: $U_{x}$ contains $x$ and at least three, but not all, elements of the space; if $x \neq y$, there exists one and only one $U_{x y} ; U_{x y}$ is simply ordered, consisting of $x, y$, and all other elements of the space between $x$ and $y ; U_{x y}$ satisfies the Dedekind cut postulate and Cantor's linearity postulate; given $p$ non-element of $U_{x y}, U_{x p} \cdot U_{x y}=x$ or $U_{x y}$. Define: $x$ is a limit point of set $M$ if $U_{x} \cdot(M-x) \neq 0$ implies $U_{x} \cdot(M-x)$ infinite. Other topological properties are defined as usual. The only connected sets are certain subsets of neighborhoods. Given set $M, K_{M}$ consists of all points $k$ of $S-M$ such that every $U_{k} \cdot M \neq 0$. This $K$-set facilitates the study of openness and closedness and methods of closing non-closed sets. The following subsets of $M$ are studied in detail: $L_{M}$ containing all points $m$ such that every $U_{m} \cdot M$ is infinite; $P_{M}$ containing remaining limit points of $M ; W_{M}$ containing all points $m$ such that every $U_{m} \cdot M$ is finite; $N_{M}$ containing remaining non-limit points of $M$. Further results are obtained by a similar classification of the elements of $S-M$ with reference to $M$. (Received July $13,1953$. )

\section{8t. I. S. Gál: On a computable looping invariant of knots.}

Let $C$ be an arbitrary knot-cycle consisting of elements $a^{\alpha}, a^{-\alpha}, b^{\beta}, b^{-\beta}, \cdots$. Let $A\left(a^{\alpha}\right)$ denote the linearily ordered sequence of elements obtained from $C$ by writing down its elements starting with $a^{\alpha}$ and proceeding with respect to a fixed orientation of $C$. Assume that the linear ordering of the elements $x^{\xi}, x^{-\xi}, y^{\eta}, y^{-\eta}$ in $A\left(a^{\alpha}\right)$ is $x^{\xi} y^{-\eta} x^{-\xi} y^{\eta}$ with $R \xi=R \eta$. Then the signature $\lambda_{x y}\left(a^{\alpha}\right)$ is defined to be $R \xi I \xi R \eta I \eta$. In all other cases the signature is defined to be 0 . Let $\Lambda\left(C, a^{\alpha}\right)=\sum \lambda_{x y}\left(a^{\alpha}\right)$ where the summation is extended over all couples $x, y$ of $C$. One can prove that $\Lambda(C)$ is independent of the special choice of $a^{\alpha}$ and is an invariant of the knot. This invariant is sufficient to distinguish all parallel knots and double knots (save for reflection) belonging to a fixed carrier knot. For example if $C_{M}$ and $C_{W}$ denote the alternating torus knot with $2 n+1$ double points and Whitehead's double knot with the circle as the carrier knot, then $\Lambda\left( \pm C_{M}\right)=n(n+1)$ and $\Lambda\left(C_{W}\right)=2 n . \Lambda=0$ does not characterize the circle, e.g. $\Lambda\left(3_{1}+4_{1}\right)=\Lambda\left(8_{21}\right)=0$. (Received July 17, 1953.)

\section{I. S. Gál: The complete characterization of knots by abstract} cycles.

It is known that a knot is completely determined by any of its regular normed projections. One can associate with any regular and normed projection an abstract cycle in the following way: Let $a, b, c, \cdots$ denote the double points of the projection and let an orientation be given. By the homeomorphism of the projection onto the unit circle there are two distinct points of the circle for every double point $a, b, c, \cdots$. The point of the circle corresponding to $a$ on the upper (lower) line segment of the projection will be denoted by $a^{1+i}$ or $a^{1-i}\left(a^{-1-i}\right.$ or $\left.a^{-1+i}\right)$ according as the crossing takes place from right to left or from left to right. For instance in the case of two trefoil knots the corresponding cycles are (for projections with three double points) $\left(a^{1+i} b^{-1-i} c^{1+i} a^{-1-i} b^{1+i} c^{-1-i}\right)$ and $\left(a^{1-i} b^{-1+i} c^{1-i} a^{-1+i} b^{1-i} c^{-1+i}\right)$. One can prove the following results: 1 . A knot is completely determined by any of its knot-cycles. 2. The (finite) 
set $\{C\}$ of all knot-cycles belonging to a fixed knot and having minimal number of elements is an invariant of the knot. (Received June 15, 1953.)

700. Leonard Gillman (p) and Melvin Henriksen: Linearly ordered paracompact spaces.

A linearly ordered space is a linearly ordered set $X$ in its interval topology. A Dedekind cut $u=(A \mid B)$, in which $A$ has no last element and $B$ no first, is called an interior gap, and is regarded as a "virtual" element satisfying $a<u<b$ for all $a \in A$ and $b \in B$. If $X$ itself has no first (last) element, a left (right) end-gapl $l(r)$ is introduced, as a virtual element satisfying $l<x(r>x)$ for all $x \in X$. Let $u$ be any gap of $X$ other than $l(r)$, and let $S=\left\{x_{\xi}\right\}_{\xi<\omega_{\alpha}}$ be an increasing (decreasing) sequence of points of $X$, in which $\omega_{\alpha}$ is a regular initial ordinal, and such that $\lim _{\xi<\omega_{\alpha}} x_{\xi}=u ; S$ is called a $Q$-sequence at $u$ if, for every transfinite limit ordinal $\lambda<\omega_{\alpha}, \lim _{\xi<\lambda} x_{\xi}$ is a gap. A gap $u$ is a $Q$-gap if there exist both an increasing $Q$-sequence at $u$ and a decreasing $Q$-sequence at $u$ (or only the appropriate one, in case $u$ is an end-gap). Theorem 1 . A linearly ordered space $X$ is paracompact if and only if every gap of $X$ is a $Q$-gap. Theorem 2. Every linearly ordered space is countably paracompact. (For definitions, see, e.g., Canadian Journal of Mathematics vol. 3 (1951) pp. 219-224.) (Received July $17,1953$. )

701t. Leonard Gillman and Melvin Henriksen: Linearly ordered Q-spaces.

Terminology is as in preceding abstract. A cardinal $\boldsymbol{\aleph}_{\alpha}$ is nonmeasurable if no countably additive two-valued measure can be defined on the family of all subsets of a set of power $\boldsymbol{N} \alpha_{\alpha}$, in such a way that every subset consisting of just one element has measure zero, and the entire set has measure one. Every $\aleph_{\alpha}$ smaller than the first strongly inaccessible cardinal $>_{\text {No }_{0}}$ is nonmeasurable (Ulam, Fund. Math. vol. 16 (1930) p. 146 ff.; Tarski, Fund. Math. vol. 30 (1938) p. 153). A $Q$-gap $u$ of a linearly ordered space is nonmeasurable if the cardinal of each $Q$-sequence at $u$ is nonmeasurable. Theorem. For every linearly ordered space $X$, the following statements are equivalent. (1) $X$ is a $Q$-space (for definition, see Hewitt, Trans. Amer. Math. Soc. vol. 64 (1948) p. 85). (2) $X$ is paracompact, and every closed subspace of $X$ is a $Q$ space. (3) $X$ is paracompact, and every closed discrete subspace of $X$ is a $Q$-space. (4) Every gap of $X$ is a nonmeasurable $Q$-gap. Corollary. Every linearly ordered $Q$-space is paracompact. Remark. (3) implies (1) for arbitrary Hausdorff spaces, not only for those that are linearly ordered (Katětov, Fund. Math. vol. 38 (1951) pp. 73-84; Shirota, Osaka Math. J. vol. 4 (1952) pp. 23-40). (Received July 17, 1953.)

702. Leonard Gillman, Melvin Henriksen (p), and Meyer Jerison: On a theorem of Gelfand and Kolmogoroff.

Let $C$ denote the ring of all continuous real-valued functions on a completely regular space $X$. Theorem 1 . The $m$-closure (Hewitt, Trans. Amer. Math. Soc. vol. 64 (1948)) of any ideal of $C$ is an intersection of maximal ideals. (This was conjectured by Hewitt.) Let $C^{*}$ denote the subring of bounded functions; $C^{*}$ is $m$-closed. Theorem 2. The $m$-closure of every ideal of $C^{*}$ is an intersection of maximal ideals (of $C^{*}$ ) iff $C$ $=C^{*}$. The proofs are based on the theorem of the title (stated without proof in C.R. (Doklady) Acad. Sci. URSS. vol. 22 (1939), but proved herein): $M$ is a maximal ideal of $C$ iff there is a unique $p \in \beta X$ (Stone-Cech compactification) such that $M$ is the set of all $f \in C$ for which $p \in \bar{Z}(f)$ (closure in $\beta X$ of the set of zeros of $f$ ). This theorem is further applied to give alternate constructions of Hewitt's $Q$-space $\nu X$ 
(loc. cit.), as follows. Regard $f \in C$ as a continuous function on $X$ into the one-point compactification of the reals, and denote by $\hat{f}$ its unique extension over $\beta X$. Theorem 3. $\nu X$ is the set of all $p \in \beta X$ such that for every $f \in C, \vec{f}(p) \neq \infty$. Theorem $4 . \nu X$ is the intersection of all the $Q$-spaces $Y$ such that $X \subset Y \subset \beta X$. (Received July 17,1953.)

\section{S. I. Goldberg: Representation theory of Lie algebras. II. Pre- liminary report.}

Let $L$ be a Lie algebra over a field $K$ of characteristic $0, M$ a subalgebra of $L$, and $\{V, P\}$ a representation module of $L$. Consider the groups $C^{q}(L, V)$ and $C^{q}(L, M, V)$ of $q$-dimensional cochains and relative cochains $\bmod M$ and form the quotient $C^{q}(L, V) / C^{q}(L, M, V)=C *(L, M, V)$ of reduced cochains. The author then displays the exact sequence $H^{0}(L, M, V) \rightarrow^{\imath *} \ldots \rightarrow^{\delta *} H^{q}(L, M, V) \rightarrow^{* *} H^{q}(L, \quad V)$ $\rightarrow{ }^{\pi *} H^{q}(L, M, V) \rightarrow \rightarrow^{\delta *} H^{q+1}(L, M, V) \rightarrow{ }^{\imath *} \ldots$. The following result is then obtained: Let $L$ be a semi-simple Lie algebra over $K, M$ a subalgebra of $L$, and $P$ an irreducible and nontrivial representation of $L$, then $H^{q}(L, P)=H^{q}(L, M, P)=\{0\}$ for all dimensions $q$. In the case where $V=K$ the following results are established: Let $\mathrm{g}$ be a compact connected semi-simple Lie group, $\mathfrak{h}$ a closed connected subgroup of $\mathfrak{g}$, and let $L$ and $M$ be the Lie algebras of $\mathfrak{g}$ and $\mathfrak{h}$ respectively. Then by using spectral sequences $H^{1}(M) \cong H^{2}(L, M)$ and $H^{1}(L, M)=\{0\}$. For all $L$ and $M$ over $K(L$ semisimple) and all $P, H_{*}^{1}(L, M, P) \cong H^{2}(L, M, P)$. (Received April 29, 1953.)

\section{4t. Lillian Gough: Logical relations between pairs of properties used to define endpoint.}

The logical relations between pairs of twenty-five properties which might be taken as definitions of endpoint of a connected set, continuum, or continuous curve are discussed in this paper. Properties 3 through 9 are due to H. M. Gehman (Trans. Amer. Math. Soc. vol. 30 (1928) pp. 63-84), property 10, to W. L. Ayres (Math. Zeit. (1931) pp. 161-178), properties 11, 12, 13 to R. H. Bing (Duke Math. J. vol. 18 (1951) pp. 653-663). Properties 3A, 4A, 5A, 6A, 8A, 9A, 10W, 10S, and 12A are obtained by substituting the concept of connected subsets for that of subcontinuum in the statements of the corresponding properties 3-12. Properties 11B, 12B, and 13B are concerned with a neighborhood of the point in question rather than with the entire set as are 11,12 , and 13 . In $8 \mathrm{C}$ and $8 \mathrm{D}$ the concepts of subcontinuum and connected subset replace those of irreducible subcontinuum and irreducibly connected subset of 8 and $8 \mathrm{~A}$ respectively. The properties concerned with subcontinua containing the point in question may be satisfied vacuously by a point of a connected set which is not considered an endpoint. Hence the "A" properties, property $4 \mathrm{~A}$ particularly, seem better definitions of endpoint for a set which is connected but not closed. (Received July 20, 1953.)

705. Mary-Elizabeth Hamstrom: Concerning the imbedding of $u p$ per semicontinuous collections of continua in continuous collections of continua.

In the plane, let $G$ be an upper semicontinuous collection of compact atriodic continuous curves which is an arc with respect to its elements. The collection $G$ is said to be imbedded in a collection $W$ provided it is true that each element of $G$ is contained in some element of $W$ and each element of $W$ contains one and only one element of $G$. If $H$ is a closed subcollection of $\operatorname{arcs}$ of $G, H^{(n)}$ denotes the collection of all elements $g$ of $H$ such that if $\epsilon$ is a positive number, then every segment of $G$ 
containing $g$ contains an element of $H$ which has a subarc the distance between whose endpoints is less than $\epsilon$ but whose diameter is not less than $1 / n$. The notion of a $K$-curve of $G$ is introduced and it is shown that there is a continuous collection $W$ of mutually exclusive simple closed curves such that $G$ is imbedded in $W$ if and only if (1) each element of $G$ is a $K$-curve of $G,(2)$ if $H$ is a closed subcollection of arcs of $G$ then for no natural number $n$ is it true that $H^{(n)}=H$, and (3) of each pair of simple closed curves of $G$ one is interior to the other. Certain related theorems are also proved. (Received July $8,1953$. )

\section{J. G. Hocking: 1-monotone images of 2-manifolds.}

It is the object of this note to establish the following result: Let $M$ be a 2-manifold with boundary $B$ and let $f$ be a compact, 1-monotone mapping of $M$ such that $f \mid B$ is monotone. Then $f(M)$ is homeomorphic to $M$. This is an extension (to the case of a manifold with boundary) of a theorem of Roberts and Steenrod (Theorem 1 of Monotone transformations of 2-dimensional manifolds, Ann. of Math. vol. 39 (1938) pp. 851862 ) which was first generalized by V. Martin to the noncompact case without boundary in his paper Monotone transformations of non-compact two-dimensional manifolds, Duke Math. J. vol. 8 (1941) pp. 136-153 (see Theorem 1). The method of proof is to use a characterization of 2-manifolds due to G. S. Young in order to prove that $f(M)$ is a 2-manifold. Then a simple application of V. Martin's theorem completes the argument. As an application of this result above one proves the following: Let $M$ be a compact 2-manifold with boundary $B$ and let $f$ be a quasi-interior, monotone-1 mapping of $M$ such that $f \mid B$ is monotone. Then $f$ may be approximated arbitrarily closely by light interior mappings. (Received July 17, 1953.)

\section{7t. Meyer Jerison: A property of extreme points of compact convex} sets.

Let $\left\{K_{n}\right\}$ be a uniformly bounded sequence of weak*-closed, convex sets in the conjugate space of a Banach space, and let $A_{n}$ be the set of extreme points of $K_{n}$. Then $K=\bigcap_{n=1}^{\infty} K_{n}$ is contained in the weak*-closed convex hull of the set $A$ $=\lim \sup A_{n}$ (in the topological sense). If, in addition, $\left\{K_{n}\right\}$ is a decreasing sequence, then $A$ contains all of the extreme points of $K$. (Received July 13,1953.)

708. Leo Katz (p) and J. H. Powell: On the number of locally restricted directed graphs.

A directed graph on $n$ nodes $P_{i}, i=1,2, \cdots, n$, is locally restricted in the sense that the numbers $r_{i}$, of joins $P_{i} \rightarrow x$, and $s_{i}$, of joins $y \rightarrow P_{i}$, are specified. The vectors $r$ and $s$ are two $n$-part partitions of the total number of joins. Using a matrix representation of the graph, it is shown that the number of directed graphs so restricted is expressible in terms of certain bipartitional functions tabulated by P. V. Sukhatme (Philos. Trans. Roy. Soc. London Ser. A vol. 237 (1938) pp. 375-409), and earlier by Cayley (Philos. Trans. Roy. Soc. London vol. 147 (1857) pp. 489-499). Sukhatme gives $A g(s \mid r)$, the number of ways to fill the cells of a rectangular lattice so that the column sums form the partition $s$ and the row sums, the partition $r$. The number of locally restricted directed graphs is $\eta(s \mid r)=A g\left\{\prod_{1}^{n}\left(1+\delta_{i}\right)^{-1}(s \mid r)\right\}$, where $A g$ is linear in the partitions, $\delta_{i}$ operates on a pair of partitions to reduce the $i$ th components of each by unity, $\left(1+\delta_{i}\right)^{-1} \equiv 1-\delta+\delta^{2}-\delta^{3}+\cdots$ and $A g\left(S^{(\alpha)} \mid r^{(\alpha)}\right)=0$ if any of the $(2 n)$ arguments is negative. This work was sponsored by the Office of Naval Research. (Received July $16,1953$. ) 
709t. S. D. Liao: On fourth obstruction of sphere bundles. Preliminary report.

As application of certain homotopy properties of cyclic products of spheres (Liao, On topology of cyclic products of spheres, Bull. Amer. Math. Soc. Abstract 59-5-555), some formulas for fourth obstruction of sphere bundles may be deduced, using technique similar to that for the case of secondary obstructions (Bull. Amer. Math. Soc. Abstract 58-4-470). Let $\mathcal{B}=\{B, E, \phi\}$ be a bundle with base space $B$ a connected complex, total space $E$, projection $\phi$, and fiber an $n$-sphere $S_{n}$. Assume that the fundamental group of $B$ acts trivially on the $n$th homotopy groups of the fibers. Write the Gysin sequence of $\mathcal{B}$ as $\cdots \rightarrow H^{s}(B, J) \rightarrow^{\phi *} H^{s}(E, J) \rightarrow^{\beta} H^{s-n}(B, J)$ $\rightarrow H^{s+1}(B, J) \rightarrow \cdots$ where $J$ denotes the group $Z$ of integers or the group of integers $\bmod p$. Suppose that the primary obstruction of $B$ vanishes. Then, $\phi^{*}$ is an intoismorphism. Let $f$ be a cross section of $\mathbb{B}$ over $B_{n}$ ( $=$ the $n$th skeleton of $B$ ) which extends over $B_{n+3}$. Then, for the fourth obstruction $Z^{n+4}(f) \bmod 2$ and $\bmod 3$, we have:

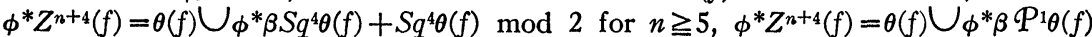
$-\mathcal{P}^{1} \theta(f) \bmod 3$ for $n \geqq 3$ and $n \neq 4$, where $\theta(f)=a-\phi^{*} f^{*} a$ with $a \in H^{n}(E, Z)$ satisfying the condition $\beta(a)=$ the unit of the integral cohomology ring of $B$, and $P^{1}$ is the cyclic reduced power operation mod 3. (Received July 8, 1953.)

\section{E. A. Michael: A selection theorem for continuous functions.}

Let $X$ and $Y$ be metric spaces, $Y$ complete, let $F$ be a function from $X$ to the nonempty subsets of $Y$, and let $n$ and $k$ denote integers $\geqq-1 . F$ is called lower semi-continuous if $\{x \in X \mid F(x) \cap U \neq \varnothing\}$ is open in $X$ for every open $U \subset Y$. A collection $\mathbb{B}$ of subsets of $Y$ is called equi-LCn if it has the following property: If $y_{0} \in B_{0} \in \mathcal{B}$, and if $U$ is a neighborhood of $y_{0}$ in $Y$, then there exists a neighborhood $V$ of $y_{0}$ in $Y$ such that, for every $k \leqq n$ and every $B \in \mathcal{B}$, every continuous mapping of the $k$-sphere into $B \cap V$ is homotopic to a constant mapping in $B \cap U$. The following theorem, which is related to results of Kuratowski [Fund. Math. vol. 24 (1935) p. 269], Montgomery and Zippin [Amer. J. Math. vol. 65 (1943) Theorem 3.1], and the author [Bull. Amer. Math. Soc. Abstract 59-2-262], can now be proved. Let $F$ be lower semicontinuous with $\{F(x)\}_{x \in X}$ equi-LC ${ }^{n}$, and let $A$ be a closed subset of $X$ with Lebesgue $\operatorname{dim}(X-A) \leqq n+1$. Then every continuous $g: A \rightarrow Y$, such that $g(x) \in F(x)$ for every $x \in A$, can be extended to a continuous $f: U \rightarrow Y, U$ a neighborhood of $A$, such that $g(x) \in \overline{F(x)}$ for every $x \in U$. If, moreover, every $F(x)$ has all its homotopy groups of order $\leqq n$ vanishing, then one can take $U=X$. (Received July 17,1953 .)

711. E. E. Moise: Affine structures in 3-manifolds. VII. Disks which are pierced by intervals.

Let $D$ be a disk, and let $e$ be an arc, in a triangulated 3-manifold $K$, such that $D \cap e$ is a point $p$ which is an interior point both of $D$ and of $e$. Suppose that $e$ pierces $D$ at $p$, in the serise that for each sufficiently small open neighborhood $U$ of $p, U-D$ is the sum of two disjoint open sets each of which intersects the component of $U$ ) $e$ that contains $p$. Theorem 1 . If $D$ and $e$ are tamely imbedded, then so also is $D \cup_{e}$. Theorem 2. If $D$ is locally polyhedral except at $p$, and $e$ is a polyhedron, then $D \cup_{e}$ is tamely imbedded. (Received July 17, 1953.)

712t. Mabel D. Montgomery: Axioms of topology.

The subject of this paper is a set of twelve axioms based on limit point as undefined concept. These axioms, referred to as the $L$-axioms, are expressed in terms of the rela- 
tionship $p L A$ where $p$ is a point and $A$ is a subset of a space $S$. The $L$-axioms do not form an independent set. Weaker forms of certain axioms are included in order to determine the properties of the relation $p L A$ which are sufficient to yield most of the fundamental theorems of topology. It is shown that a certain four of the axioms form an independent set equivalent to the complete set. The question of equivalence of the $L$-axioms to some other sets of axioms for topology is considered. It is established that the set of $L$-axioms is equivalent to the first three Kuratowski axioms but is not equivalent to any subset of the Riesz or Hausdorff axioms. A weak upper semicontinuous decomposition of a space $M$ and its associated decomposition space $M^{*}$ are defined, and it is proved that if $M$ satisfies the $L$-axioms, so also does $M^{*}$. (Received July 17, 1953.)

\section{3t. R. S. Pierce: Coverings of. a topological space.}

Let $P$ be a semi-lattice. Definition: a $P$-space is a set $S$ of proper dual ideals of $P$, which is topologized by taking the sets of the form $\{X \in S \mid a \in X\}(a \in P)$ as a neighborhood basis. Definition: a finite nonempty subset of $P$ is called a $P$-covering. A $P$-covering ideal is a nonempty set $\mathfrak{a}$ of $P$-coverings such that (1) if $\alpha, \beta \in \mathfrak{a}$, then $\alpha \wedge \beta=\{a \wedge b \mid a \in \alpha, b \in \beta\} \in \mathfrak{a}$ and (2) if $\alpha \in \mathfrak{a}$ and $\alpha$ refines $\beta$ (that is, for any $a \in \alpha$, there exists $b \in \beta$ with $a \leqq b)$, then $\beta \in \mathfrak{a}$. For any compact $T_{1} P$-space $S$, denote by $\mathfrak{a}(S)$ the set of all $P$-coverings $\alpha$ which satisfy $\alpha \bigcap X \neq \varnothing$ for all $X \in S$. If $\mathfrak{a}$ is a $P$ covering ideal, let $S(\mathfrak{a})$ be the $P$-space of all dual ideals which are minimal in the set of dual ideals $D$ satisfying $D \bigcap \alpha \neq \varnothing$ for all $\alpha \in \mathfrak{a}$. Then $\mathfrak{a} \rightarrow S(\mathfrak{a})$ and $S \rightarrow \mathfrak{a}(S)$ are reciprocal correspondences between the set of all $P$-covering ideals and the set of all compact $T_{1} P$-spaces. (Received July 20,1953.)

\section{Everett Pitcher: Chain groups and critical point inequalities.}

The construction of a lattice of exact sequences from filtered chain groups and a boundary homomorphism is inverted as follows. Suppose $\left(^{*}\right) \cdots \rightarrow A_{r}^{t} \rightarrow B_{r}^{t} \rightarrow C_{r}$ $\rightarrow A_{r-1}^{t} \rightarrow \cdots, r=\cdots,-1,0,1, \cdots$, is an exact sequence of discrete [or compact] abelian groups, $t=1, \cdots, N$, and suppose $A_{0}^{1}=0, A_{r}^{t}=B_{r}^{t-1}$. Examples: $X_{1} \subset \cdots$ $C X_{N}, B_{r}^{t}=H_{r}\left(X_{t}\right)$ [or $\left.\pi_{r}\left(X_{t}\right)\right], C_{r}^{t}=H_{r}\left(X_{t}, X_{t-1}\right)$ [or $\pi_{t}\left(X_{t}, X_{t-1}\right)$ ]; in critical point theory of $f: X \rightarrow R, X_{t}=\{x \mid f x \leqq a(t)\}$ where $a(t)$ is increasing sequence separating critical values of $f$. Theorem: There are filtered chain groups $J_{r}$ obtained from $C_{r}^{1}, \cdots, C_{r}^{N}$ by successive group extensions and homomorphisms $\partial: J_{r} \rightarrow J_{r-1}$ of order 2 such that $\left({ }^{*}\right)$ is the lattice of exact sequences. In particular $(* *) B_{r}^{N}=\partial^{-1} 0 / \partial J_{r+1}$ and $J_{r} / \partial^{-1} 0=\partial J_{r}$. In the critical point application, $(* *)$ is a strengthened group theoretical form of the Morse inequalities. If all critical points are nondegenerate and if $M_{q}, R_{q}, \eta_{q}$ denote the number of critical points of index $q$ and the Betti number and number of torsion coefficients in dimension $q$ (integer coefficients), then $M_{q} \geqq R_{q}+\eta_{q}+\eta_{q-1}$ and $M_{q}-M_{q-1}+\cdots+(-1)^{q} M_{0} \geqq R_{q}-R_{q-1}+\cdots+(-1)^{q} R_{0}+\eta_{q}$. (Received July 20 , 1953.)

715. Louis Robinson: An extension of the Kneser inequality to complexes and a generalization of the concept of the degree of a mapping.

There is the theorem of H. Kneser (Math. Ann. vol. 103 (1930) p. 347) which states that if $S_{p}$ and $S_{q}$ are orientable closed two-dimensional manifolds of genus $p$ and $q$ respectively with $p \geqq q \geqq 1$, then the degree $c$ of a continuous mapping of $S_{p}$ into $S_{q}$ satisfies the inequality $(p-1) \geqq|c|(q-1)$. In this paper there is proved a generalization of the Kneser inequality to certain classes of complexes. Let $K$ and $\bar{K}$ be 
connected complexes whose fundamental groups $G$ and $\bar{G}$ are each given by a finite system of generators and one essential relation. To the groups $G$ and $\bar{G}$, so given, there correspond the groups $G_{1}{ }^{*}$ and $\bar{G}_{1}{ }^{*}$ introduced by $\mathrm{H}$. Hopf (Comment. Math. Helv. vol. 14 (1941) p. 257) each of which is infinite cyclic or else consists of the identity element alone. A simplicial mapping of $K$ into $\bar{K}$ induces a homomorphism of $G_{1}{ }^{*}$ into $\bar{G}_{1}{ }^{*}$ which uniquely determines an integer which is called the pseudo-degree of the mapping. The generating element of $G_{1}{ }^{*}$ determines an integer which is called the pseudo-genus number of $K$. It is shown that between the pseudo-degree $c$ and the pseudo-genus numbers $p$ and $q$ with $p \geqq q \geqq 1$ there exists the inequality $(p-1)$ $\geqq|c|(q-1)$. (Received July 8,1953 .)

716t. Hans Samelson: A connection between Whitehead and Pontryagin products.

Let $X$ be a 1-connected topological space, and let $\Omega$ be the space of loops in $X$, with base point $x_{0}$. Let $[$,$] denote Whitehead product, as usual, and let *$ denote Pontryagin product in $\Omega$ [cf. e.g. R. Bott and H. Samelson, On the Pontryagin product in spaces of paths, to appear in Comment. Math. Helv.]. Let $\tau$ denote the composition of the standard isomorphism (lowering dimension by one) between the homotopy groups of $X$ and of $\Omega$ with the natural map of the homotopy groups of $\Omega$ into the homology groups of $\Omega$. With $\alpha \in \pi_{p+1}(X)$ and $\beta \in \pi_{q+1}(X)$ the following formula is shown to hold: $\tau[\alpha, \beta]= \pm\left(\tau \alpha * \tau \beta-(-1)^{p q} \tau \beta * \tau \alpha\right)$; special cases of this have been known to Hurewicz, Serre, G. W. Whitehead. One of the proofs uses the algebraic structure of the Pontryagin ring associated with a space consisting of two spheres joined at a point, as determined in the reference above. (Received March 31, 1953.)

\section{D. E. Sanderson: Isotopic deformation in 3-manifolds.}

If $M$ and $K$ are triangulated $n$-manifolds with boundary, a piecewise linear homeomorphism (p.l.h.) $h: M \rightarrow K$ maps simplexes of a subdivision of $M$ linearly onto simplexes of a subdivision of $K$. A piecewise linear isotopy (p.l.i.) between two p.l.h.'s $h$ and $h^{\prime}$ is a mapping $G(x, t)$ of $M \times[0,1]$ into $K$, such that $G(x, 0)=h(x), G(x, 1)$ $=h^{\prime}(x)$, and for every $t \in[0,1], G(x, t)$ is a p.l.h. If $M \subset K$ and $h$ is the identity, $G$ is an isotopic deformation of $M$. A shellable partition of $M$ is a collection of closed ordered $n$-cells $\left\{C_{i}\right\}$ with disjoint interiors, where $M=\sum C_{i}$ and $C_{i} \cap \mathrm{Bd}\left(\overline{K-\sum_{j=1}^{i-1} C_{j}}\right)$ $=\mathrm{Bd}\left(C_{i}\right)$ or is an $(n-1)$-cell $(\mathrm{Bd}(S)$ consists of points in $S$ having no neighborhood homeomorphic to $\left.E_{n}\right)$. It is shown that if $M$ is a 3-cell with triangulation $T$, and $M \cap \mathrm{Bd}(K)=\mathrm{Bd}(M)$ or is an $(n-1)$-cell, there is a subdivision of $T$ whose (closed) simplexes form a shellable partition of $M$. There exist p.l.i.'s deforming $M$ onto any simplex of $T$, or $K$ onto $\overline{K-M}$. A 3-manifold $N$ is a 3-sphere if and only if, for some 3cell $C \subset N, \overline{N-C}$ has a finite shellable partition. There are exactly two isotopy classes of homeomorphisms of $E_{3}$ onto itself. Extensive use is made of articles by E. E. Moise (Ann. of Math. vol. 56 (1952) pp. 96-114 and vol. 55 (1952) pp. 172-176, 203-214) and S. S. Cairns (Ann. of Math. vol. 45 (1944) pp. 207-219). (Received July $17,1953)$.

\section{8t. A. D. Wallace: $A$ structure theorem for compact mobs.}

Recall that a mob is an associative map $m: S \times S \rightarrow S$ such that $S$ is Hausdorff. Let $S$ be compact, let $E$ be the set of idempotents of $S$, and let $P$ be the set of all those $x \in S$ such that $x S=S$. Recall that each $e \in E$ is the unit of a unique maximal algebraic subgroup of $S$ with $e$ as unit. It is shown that $P$ is closed, that $P=\bigcup\{H(e) \mid e$ 
$\in E \cap P\}$, and that $E \cap P$ is the set of left units of $S$. Further $x y \in P$ implies $x \in P$ and $y \in P$, and $x a=x b$ implies $a=b$ if $x \in P$ and $a, b \in S$. Finally, if $e_{0} \in E \cap P$, then there is a (topological) isomorphism of $H\left(e_{0}\right) \times(E \cap P)$ onto $P$. This last result is close to an algebraic theorem of $\mathrm{H}$. B. Mann. In proving the above we use some results of R. J. Koch. Let $G$ be a compact Hausdorff group and $X$ a compact Hausdorff space. In $X$ define $x y=y$ for all $x, y$. If $S=G \times X$, then $P(S)=S, E(S)=a \times X$ ( $a$ the neutral element of $G)$ and $H(a, x)=G \times x, x \in X$. This shows that the structural results above are "best possible" when $P(S)=S$, as noted by Mann. (Received December 15, 1952.)

\section{9t. A. D. Wallace: Geometric theorems on clans.}

A clan is a compact connected Hausdorff semigroup with unit. We use the Alexander-Kolmogoroff cohomology groups as developed by Spanier. Let $S$ be a clan. If for some $p>0$ and some coefficient group $G$ we have $H^{p}(S, G) \neq 0$ but $H^{p}(A, G)=0$ for each closed proper subset $A$ of $S$, then $S$ is a (topological) group. In particular a (classical) $n$-manifold is a clan if and only if it is a group. From a celebrated result of E. Cartan we see that an $n$-sphere is a clan only for $n=1,3$. The results stated above are particularly concrete applications of general theorems. Among other consequences of these general theorems is the fact that a compact connected Hausdorff lattice is cohomologically trivial. This generalizes (in part) a result of Aumann and Eckmann. It is also shown that if an $n$-cell is a clan then the set of elements with inverses lie on the bounding $(n-1)$-sphere. (Received December 15, 1952.)

720t. C. T. Yang: On theorems of Borsuk-Ulam, Kakutani-YamabeYujobô, and Dyson.

The results of this paper mainly rely on the concept of the order of a hyperplane which is a direct generalization of the order of a point as given in Alexandroff-Hopf, Topologie. Because of this concept, several theorems on spheres, such as BorsukUlam's theorem (Fund. Math. (1933)), Kakutani-Yamabe-Yujobô's theorem (Osaka Math. J. (1950)), Dyson's theorem (Ann. of Math. (1951)), etc., are generalized. One generalization of Dyson's theorem is as follows: Let $X$ be a compact subset of a euclidean space, symmetric with respect to an $s$-plane $\Theta$. If there is an $n$th $\Theta$-symmetric homology class $(\bmod 2)$ of $X$ with respect to which the order of $\Theta$ is not zero, then for any continuous function $f$ on $X$ there are $n$ points $x_{1}, \cdots, x_{n}$ of $X$ for which $f\left(x_{1}\right)=\cdots=f\left(x_{n}\right)=f\left(x_{1}^{\prime}\right)=\cdots=f\left(x_{n}^{\prime}\right)$ and the $(s+1)$-planes $\Theta x_{1}, \cdots, \Theta x_{n}$ are mutually orthogonal, where $x_{i}^{\prime}$ is the $\Theta$-symmetric point of $x_{i}$ and $\Theta x_{i}$ is the (s+1)plane determined by $\Theta$ and $x_{i}, i=1, \cdots, n$. In particular, if $X$ is an $n$-sphere with center $\Theta$, this gives Dyson's theorem on $n$-sphere. (Received June 22,1953.)

\section{W. Cohen, Associate Secretary}

